\title{
Process Equipment Cost Estimation Final Report
}

\author{
January 2002
}

\section{H.P. Loh}

U.S. Department of Energy

National Energy Technology Laboratory

P.O. Box 10940, 626 Cochrans Mill Road

Pittsburgh, PA 15236-0940

and

P.O. Box 880, 3610 Collins Ferry Road

Morgantown, WV 26507-0880

and

Jennifer Lyons and Charles W. White, III

EG\&G Technical Services, Inc.

3604 Collins Ferry Road, Suite 200

Morgantown, WV 26505
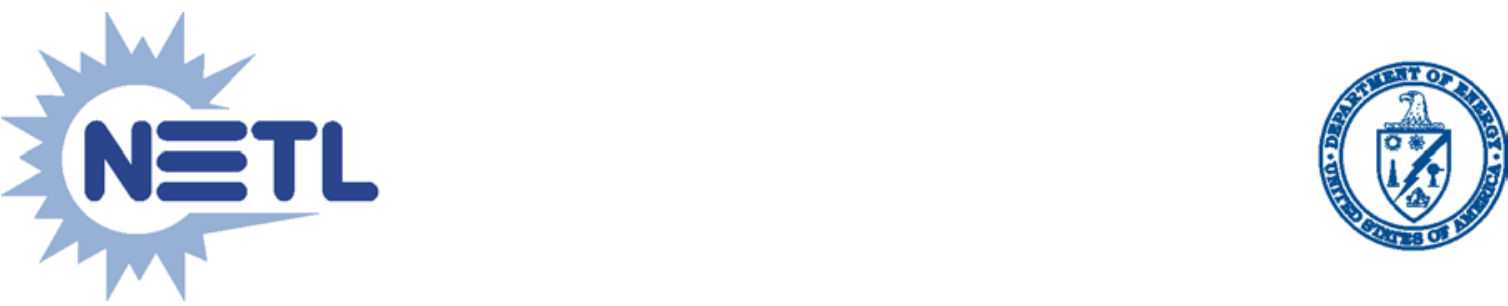


\section{Disclaimer}

This report was prepared as an account of work sponsored by an agency of the United States Government. Neither the United States Government nor any agency thereof, nor any of their employees, makes any warranty, express or implied, or assumes any legal liability or responsibility for the accuracy, completeness, or usefulness of any information, apparatus, product, or process disclosed, or represents that its use would not infringe privately owned rights. Reference herein to any specific commercial product, process, or service by trade name, trademark, manufacturer, or otherwise does not necessarily constitute or imply its endorsement, recommendation, or favoring by the United States Government or any agency thereof. The views and opinions of authors expressed herein do not necessarily state or reflect those of the United States Government or any agency thereof. 


\section{Contents}

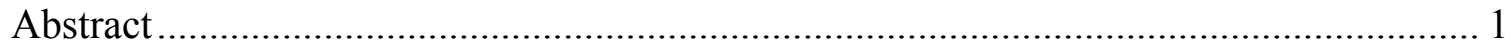

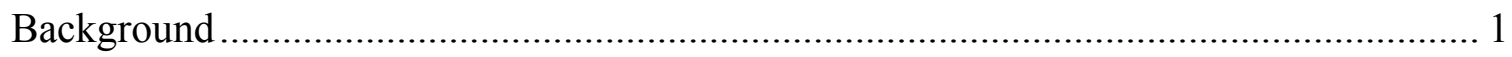

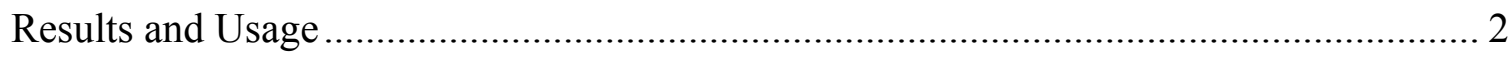

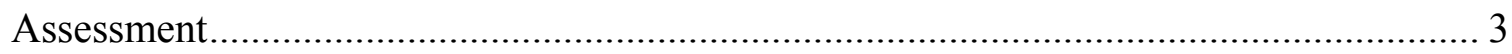

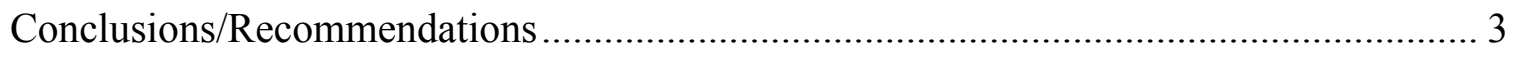

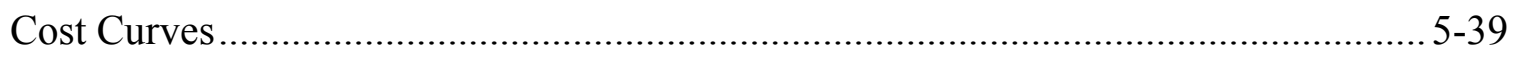

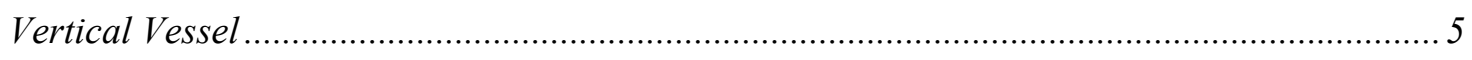

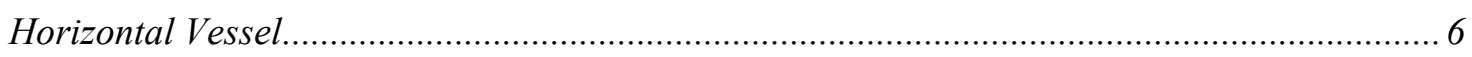

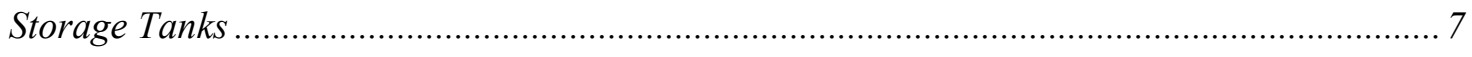

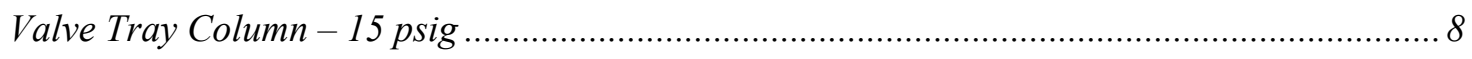

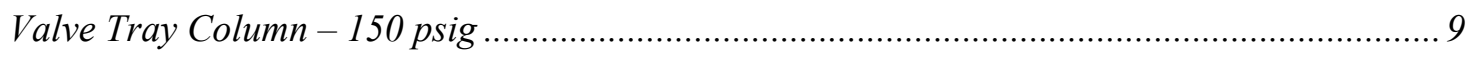

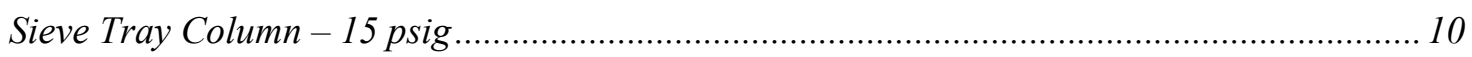

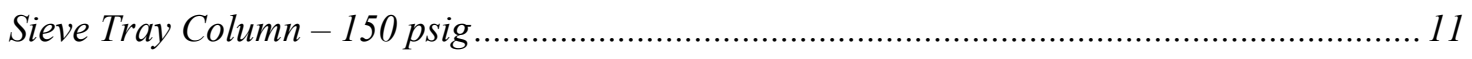

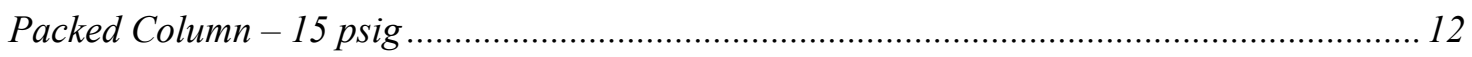

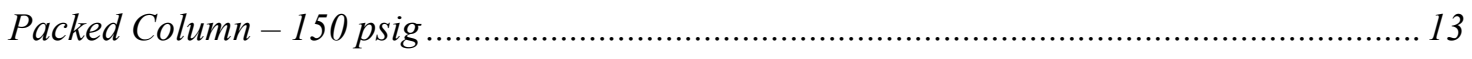

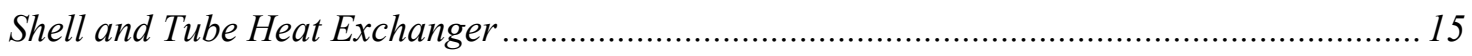

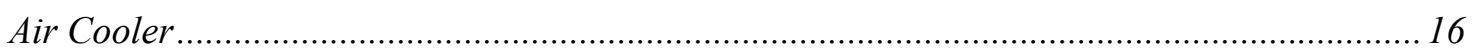

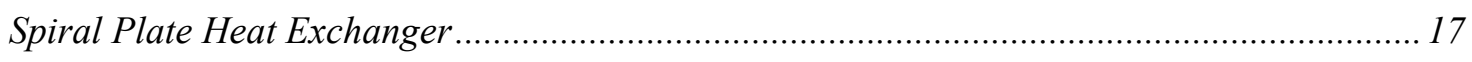

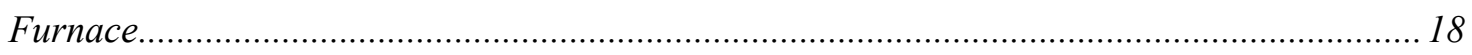

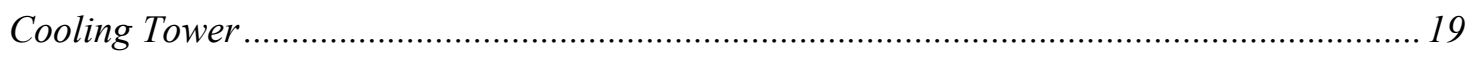

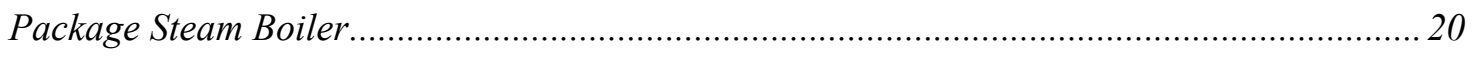

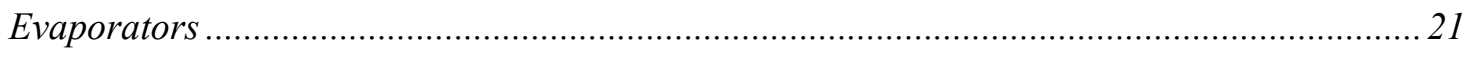

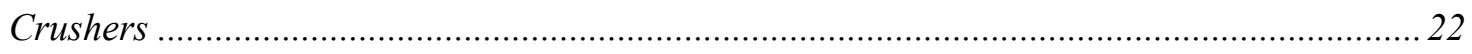

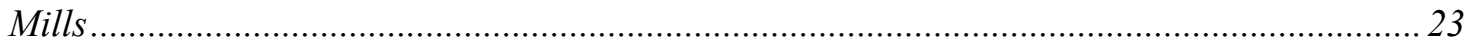

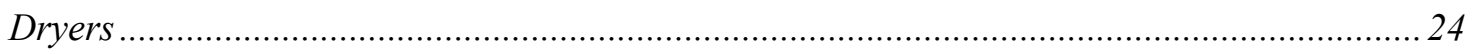

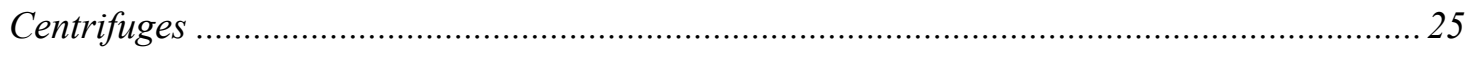

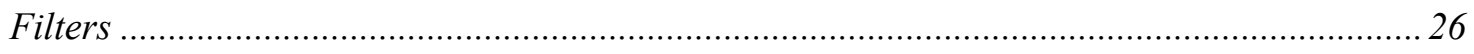

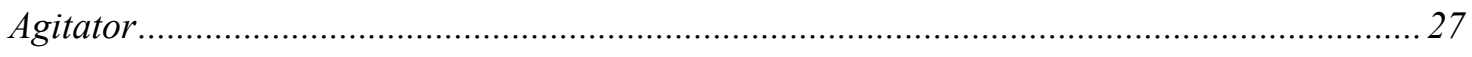

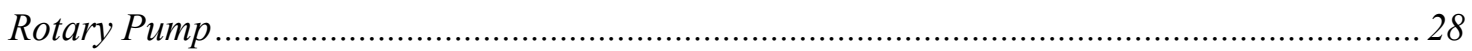




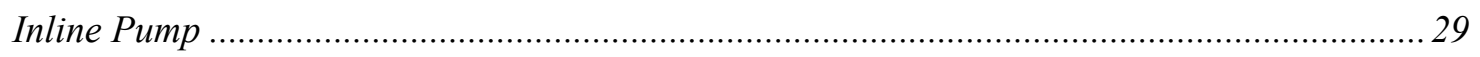

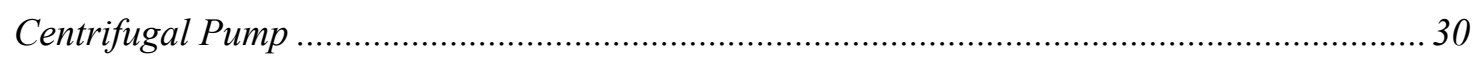

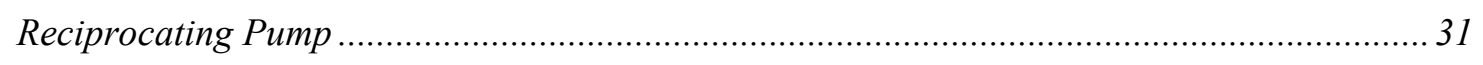

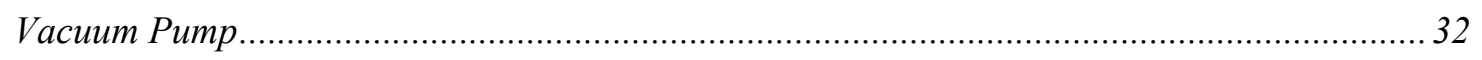

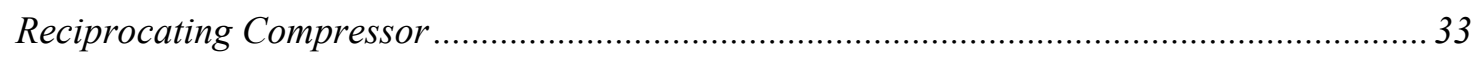

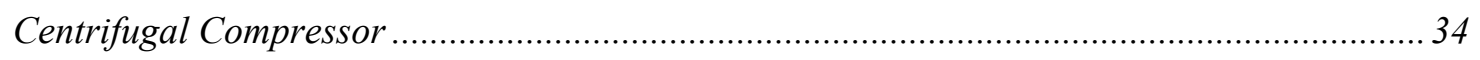

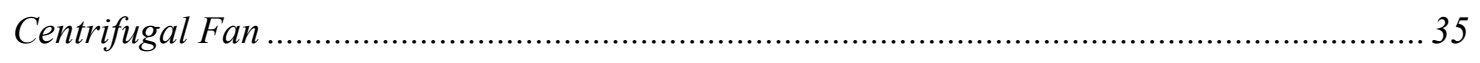

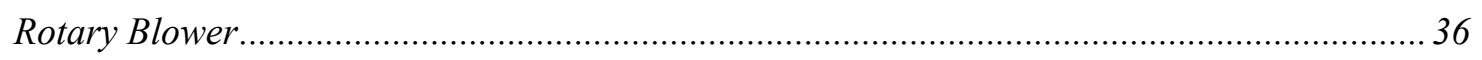

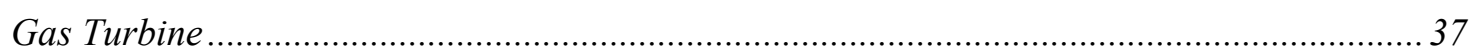

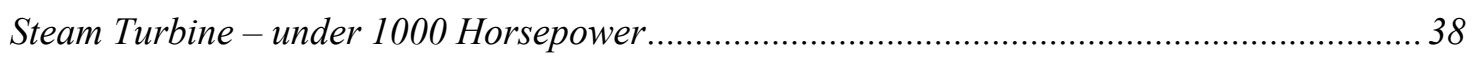

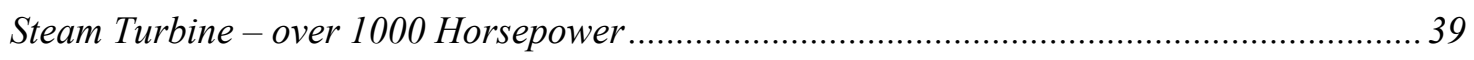

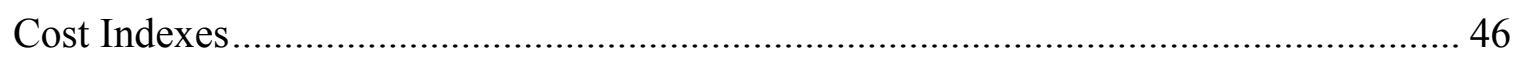

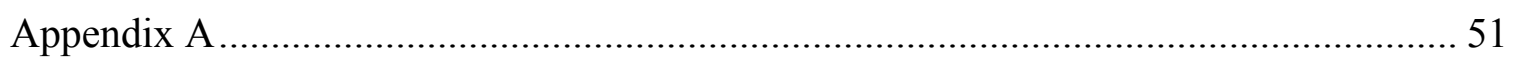

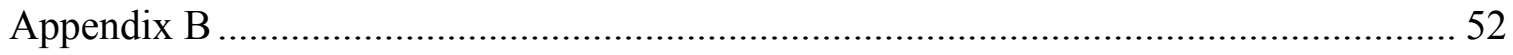




\section{List of Tables}

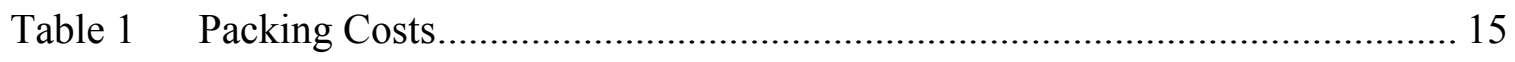

Table 2 Distributive Factors for Bulk Materials - Solids Handling Processes ........... 40

Table 3 Distributive Factors for Bulk Materials - Solids - Gas Processes .................. 41

Table 4 Distributive Factors for Bulk Materials - Liquid and Slurry Systems ........... 42

Table 5 Distributive Factors for Bulk Materials - Gas Processes .............................. 43

Table 6 Distributive Labor Factors for Setting Equipment ..................................... 44

Table 7 Factors for Converting Carbon Steel to Equivalent Alloy Costs................... 45

Table 8 Engineering News Record Construction Cost Index .................................. 47

Table 9 Marshall and Swift Installed-Equipment Index ........................................ 48

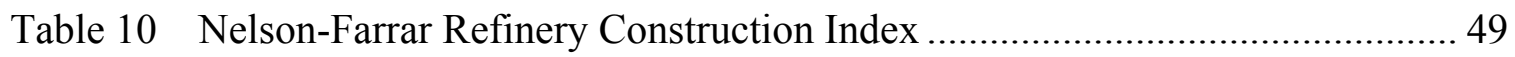

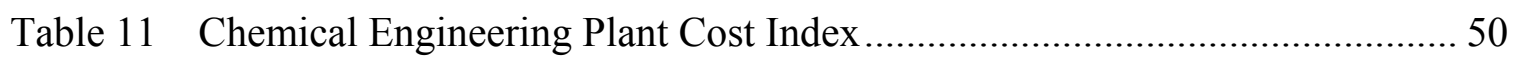




\begin{abstract}
This report presents generic cost curves for several equipment types generated using ICARUS Process Evaluator. The curves give Purchased Equipment Cost as a function of a capacity variable. This work was performed to assist NETL engineers and scientists in performing rapid, order of magnitude level cost estimates or as an aid in evaluating the reasonableness of cost estimates submitted with proposed systems studies or proposals for new processes. The specific equipment types contained in this report were selected to represent a relatively comprehensive set of conventional chemical process equipment types.
\end{abstract}

\title{
Background
}

As part of its mission to identify and develop practical and viable processes for power production, chemicals processing, fuel processing, $\mathrm{CO}_{2}$ capture and sequestration, and other environmental management applications, NETL engineers and scientists need to both perform order of magnitude cost estimates and evaluate and assess cost estimates contained in proposals for novel processes. In these applications where process and technological specifics are lacking, detailed cost estimates are not justified. Rather, rough estimates that can be obtained relatively quickly are more suitable. There are a number of tools available to NETL engineers to assist in the performance and evaluation of chemical process equipment cost estimates.

One such tool is ICARUS Process Evaluator (IPE). IPE is a sophisticated and industryaccepted software tool for generating cost estimates, process facility designs, and engineering and construction schedules. The IPE equipment library contains over 320 process equipment types. Sizing is performed using common engineering methodologies from intrinsic sizing algorithms. IPE utilizes self-contained equipment, piping, instrumentation, electrical, civil, steel, insulation, and paint sizing and design algorithms for a preliminary equipment model that is properly integrated and evaluated for many safety and operability issues.

When used with appropriate values for the adjustable design and construction parameters, IPE provides a highly detailed and accurate cost estimate. However, the program is very complex and both expensive and time consuming to learn and use. Furthermore, IPE requires well-defined process configuration and process parameters that typical proposals do not provide. In general, it is not practical or cost-effective to use IPE for the assessment of cost estimates contained in proposals for novel processes or in generating rough cost estimates from laboratory scale data. Instead, the factored estimation methodology, a cost-effective methodology widely used in industry, is more suitable for that application. To leverage the cost information contained within IPE, a series of cost curves for different equipment types were generated. The cost curves and other information contained in this report can then be used to develop the overall process plant capital cost using the factored estimation methodology. 


\section{Results and Usage}

For this activity, a general file was created in ICARUS Process Evaluator version 5.0 that contained several pieces of stand-alone equipment. The specific equipment types were selected by NETL and intended to represent a relatively comprehensive set of conventional chemical process equipment types that might be encountered in processes relevant to $\mathrm{CO}_{2}$ capture and sequestration. Each piece of equipment was then varied in size to generate costs for a spectrum of sizes. The cost versus sizing capacity was plotted for each equipment type. The data was then regressed to provide smoothed cost curves.

The cost curves for the 31 different types of equipment examined in this report are shown on pages 6 - 40. In addition to the graphs, the applicable design specifications and equipment descriptions are provided as appropriate.

All graphs portray purchased equipment cost data. This total material cost includes:

- Internals, shells, nozzles, manholes, covers, etc as noted for each piece equipment.

- Vendor engineering, shop drawings shop testing, certification.

- Shop fabrication labor (and field labor if field-fabricated).

- Typical manuals, small tools, accessories.

- Packaging for shipment by land.

- FOB Vendor.

The total material cost does not include:

- Owner/contractor indirects (engineering, shop inspection, start-up/commissioning).

- Packaging for overseas/air shipment, modularization.

- Freight, insurance, taxes/duties

- Field setting costs (off-loading, storage, transportation, setting, testing)

- Installation bulks

The total capital cost of each piece of equipment includes material and labor charges. The material charges include the delivered equipment costs and installation bulk material costs. The labor charges include labor for handling and placing bare equipment and labor for installation of bulk materials.

Installation bulks consist of foundations, structural steel, buildings, insulation, instruments, electrical, piping, painting and miscellaneous. Tables 2 - 5 list distributive percentage factors that can be used to estimate installation bulk labor and materials for different plant types. ${ }^{1}$ The factors vary depending on the type of process and the temperature and pressure of the system. The bare equipment cost is used as the base to apply the percentage factor for the installation material cost. This installation material cost is then used as the base to apply the percentage factor for determining the associated labor cost involved.

Handling and placing equipment involves unloading, uncrating, mechanical connection, alignment, storage, inspection, and other factors. The costs vary by type and size of

\footnotetext{
${ }^{1}$ AACE Recommended Practices and Standards - "Conducting Technical and Economic Evaluations in the Process and Utility Industries," adopted November 1990.
} 
equipment. The setting costs can be estimated by using historical work hours or by applying factors for labor cost as a percentage of delivered equipment cost. Table 6 shows approximate factors for setting various types of equipment. ${ }^{1}$

The total cost for installing a piece of equipment would be the bare equipment cost plus the setting labor cost plus the installation bulks material and labor costs as determined from the distributive labor percentages. See Appendix A for a detailed example.

Appendix B shows the ICARUS generated purchased/ installed costs of the equipment used in each chart. All costs in this document are reported in first quarter 1998 dollars.

\section{Assessment}

The charts can be used for preliminary purchased equipment cost estimates (i.e. order of magnitude estimates with accuracy of $+50 \% /-30 \%$ and budget estimates with accuracy of $+30 \% /-15 \%$ ). Clearly, the charts are most accurate when used for the operating conditions listed as defaults for each equipment type. Nevertheless, they should provide reasonable cost estimates for conditions that contain small or moderate deviations from the assumed design conditions. Correlations to correct for deviations in some design variables, particularly pressure, are available in the literature. Peters and Timmerhaus "Plant Design and Economics for Chemical Engineers" is one such source for correction factor data. Without appropriate correction, estimates generated for conditions that deviate markedly from those used in this study should be used with caution.

Another limitation is that most of the charts give estimates for equipment manufactured from carbon steel. Conversion factors for converting the carbon steel costs to equivalent alloy costs for a few items of equipment are shown in Table $7 .^{2}$

As mentioned previously, setting costs can be estimated by using historical data or by applying factors. It should be noted that the factors do not work well for very large pieces of equipment. If available, historical work hours provide more accurate costs.

\section{Conclusions/Recommendations}

This report contains cost curves for various equipment types at specific operating temperatures and pressures. These conditions and other design parameters are listed for each equipment type. When used within the expected design conditions, the cost estimates derived from the cost curves contained in this report will provide accurate estimates. The data can also be used to provide reasonableness estimates when the actual design conditions are outside the expected values but the level of accuracy cannot be quantified.

\footnotetext{
${ }^{2}$ Perry, Robert H. , and Don W. Green, “Perry's Chemical Engineers' Handbook,” The McGraw-Hill Companies, Inc., 1999.
} 
To help quantify the error induced by large deviations in the design conditions, it is recommended that a first-order sensitivity analysis of the cost curves be performed. Another activity that could improve the range of accuracy of the charts would be to run cases with various materials of construction to show how the price is affected. If requested, additional support can be provided to expand the set of equipment types beyond those examined in this report. For example, cost data for slurry pumps and solids conveying equipment would be useful for many of the technologies at NETL. 


\section{Cost Curves}

\section{Vertical Vessel}

Description: The vertical process vessel is erected in the vertical position. They are cylindrical in shape with each end capped by a domed cover called a head. The length to diameter ratio of a vertical vessel is typically 3 to 1 . Vertical tanks include: process, storage applications liquid, gas, solid processing and storage; pressure/vacuum code design for process and certain storage vessel types; includes heads, single wall, saddles, lugs, nozzles, manholes, legs or skirt, base ring, davits where applicable.

\section{Design Basis:}

$1^{\text {st }}$ Quarter 1998 Dollars

Shell Material:

A515

(Carbon Steel Plates for pressure vessels for intermediate and higher temperature service)

Design Temperature: $650^{\circ} \mathrm{F}$

Design Pressure: $\quad 15$ psig and 150 psig

Diameter: $\quad 2.5-8$ feet

Length: $\quad 2.7-13.3$ feet

Total Weight: $\quad 1,000-7,100$ pounds

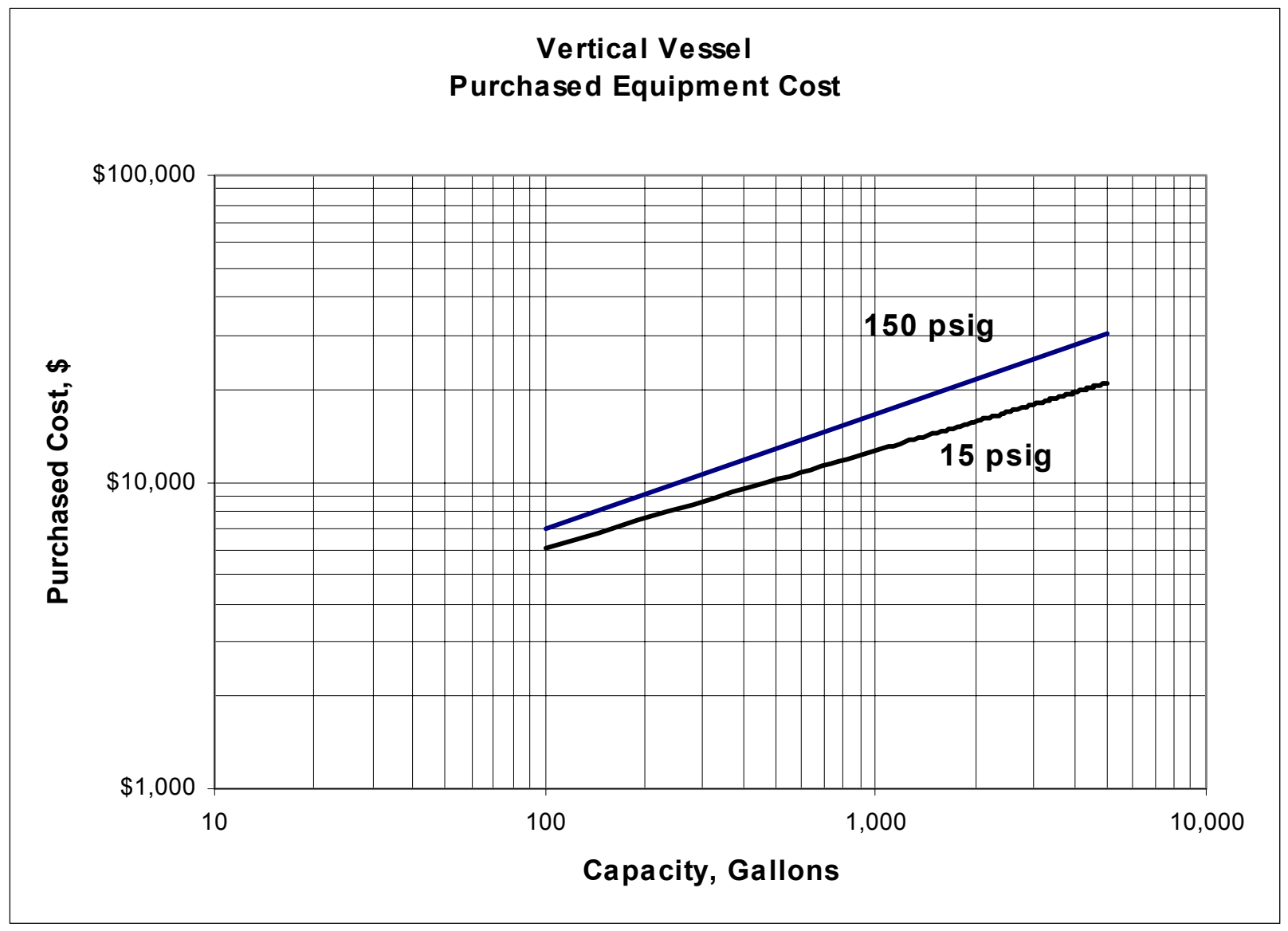




\section{Horizontal Vessel}

Description: The horizontal vessel is a pressure vessel fabricated according to the rules of the specified code and erected in the horizontal position. Although the horizontal vessel may be supported by lugs in an open steel structure, the more usual arrangement is for the vessel to be erected at grade and supported by a pair of saddles. Cylindrical, pressure/vacuum, code design and construction, includes head, single wall (base material, clad/lined), saddles/lugs, nozzles and manholes.

\section{Design Basis:}

$1^{\text {st }}$ Quarter 1998 Dollars

Shell Material: $\quad$ A515

(Carbon Steel Plates for pressure vessels for intermediate and higher temperature service)

Design Temperature: $650^{\circ} \mathrm{F}$

Design Pressure: $\quad 15$ psig

Diameter: $\quad 2-14$ feet

Length: $\quad 4.3-81$ feet

Total Weight: $\quad 1100-59,400$ pounds

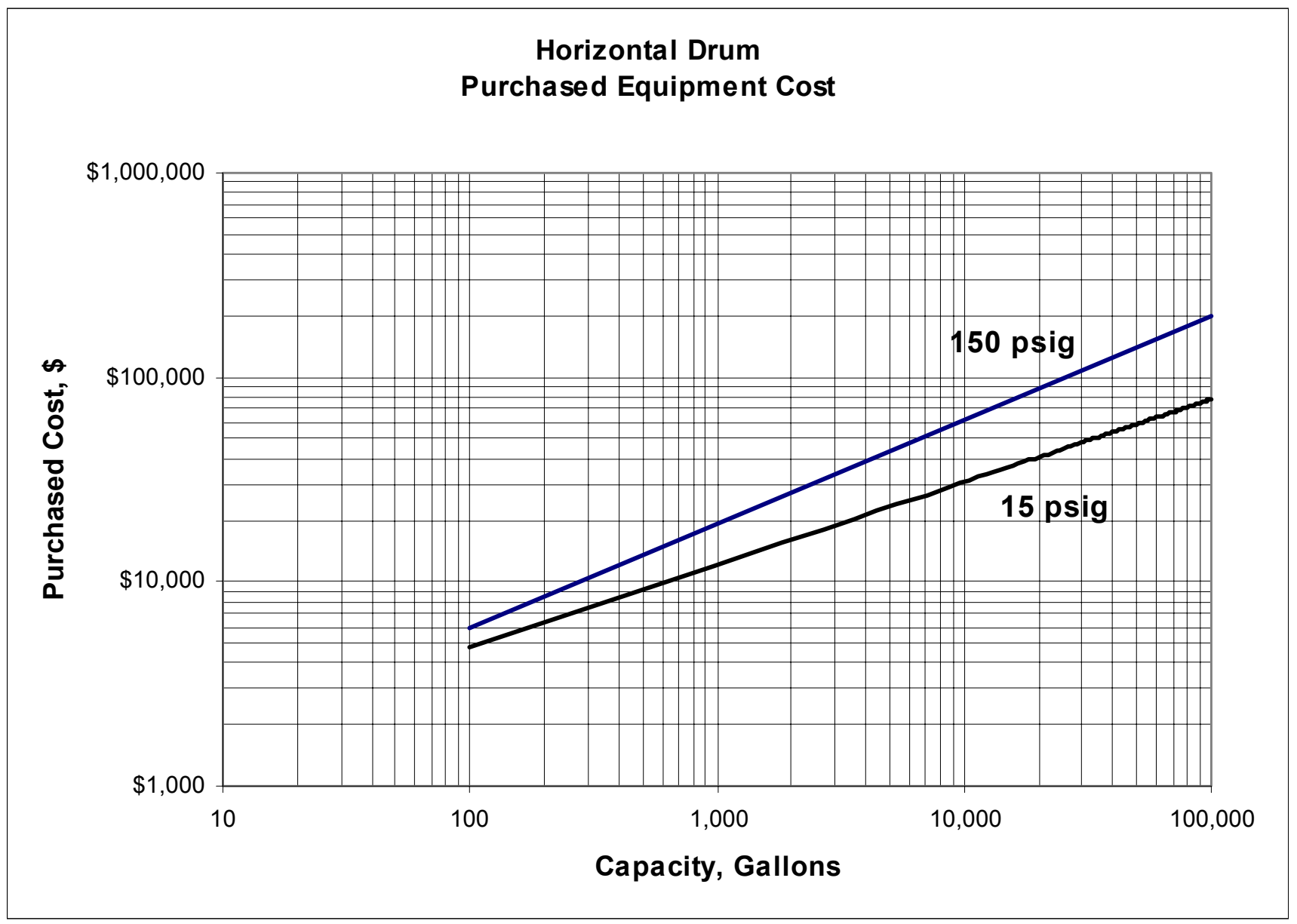




\section{Storage Tanks}

\section{Description:}

Floating Roof: Typically constructed from polyurethane foam blocks or nylon cloth impregnated with rubber or plastic, floating roofs are designed to completely contact the surface of the storage products and thereby eliminate the vapor space between the product level and the fixed roof. Floating roof tanks are suitable for storage of products having vapor pressure from 2 to 15 psia.

Cone Roof: Typically field fabricated out of carbon steel. They are used for storage of low vapor pressure (less than 2 psia) products, typically ranging from 50,000-1,000,000 gallons.

\section{Design Basis:}

$1^{\text {st }}$ Quarter 1998 Dollars

Shell Material: $\quad$ A515

(Carbon Steel Plates for pressure vessels for intermediate and higher temperature service)

Design Temperature: $650^{\circ} \mathrm{F}$

Design Pressure: 15 psig

Diameter: $\quad 2-14$ feet

Length: $\quad 4.3-81$ feet

Total Weight: $\quad 1100-59,400$ pounds

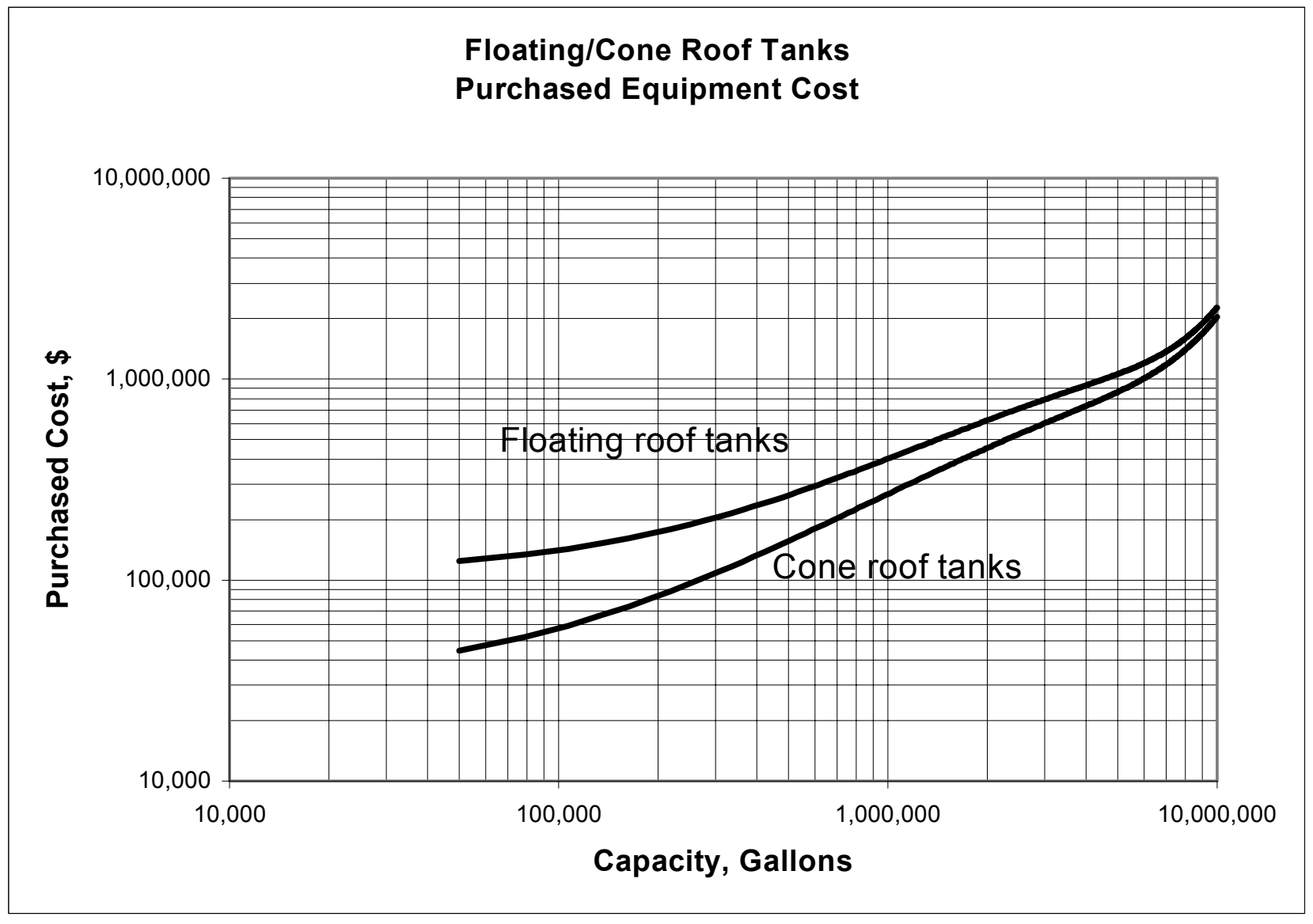




\section{Valve Tray Column - 15 psig}

Description: Pressure/vacuum column includes vessel shell, heads, single base material (lined or clad, nozzles, manholes (one manhole below and above tray stack or packed section and one manhole every tenth tray or 25 feet of packed height), jacket and nozzles for heating or cooling medium, base ring, lugs, skirt or legs; tray clips, tray supports (if designated), distributor piping, plates.

\section{Design Basis:}

$1^{\text {st }}$ Quarter 1998 Dollars

Shell Material: $\quad$ A515

(Carbon Steel Plates for pressure vessels for intermediate and higher temperature service)

Design Temperature: $650{ }^{\circ} \mathrm{F}$

Design Pressure: 15 psig

Height: $\quad 17-133$ feet

Application: Distillation

Tray Type: Valve

Tray Spacing: $\quad 24$ Inches

Tray Material: $\quad$ A285C

(Low and intermediate strength carbon steel plates for pressure vessels.)

Tray Thickness: $\quad 0.19$ Inches

\section{Single Diameter Valve Tray Column \\ 15 psig \\ Purchased Equipment Cost}

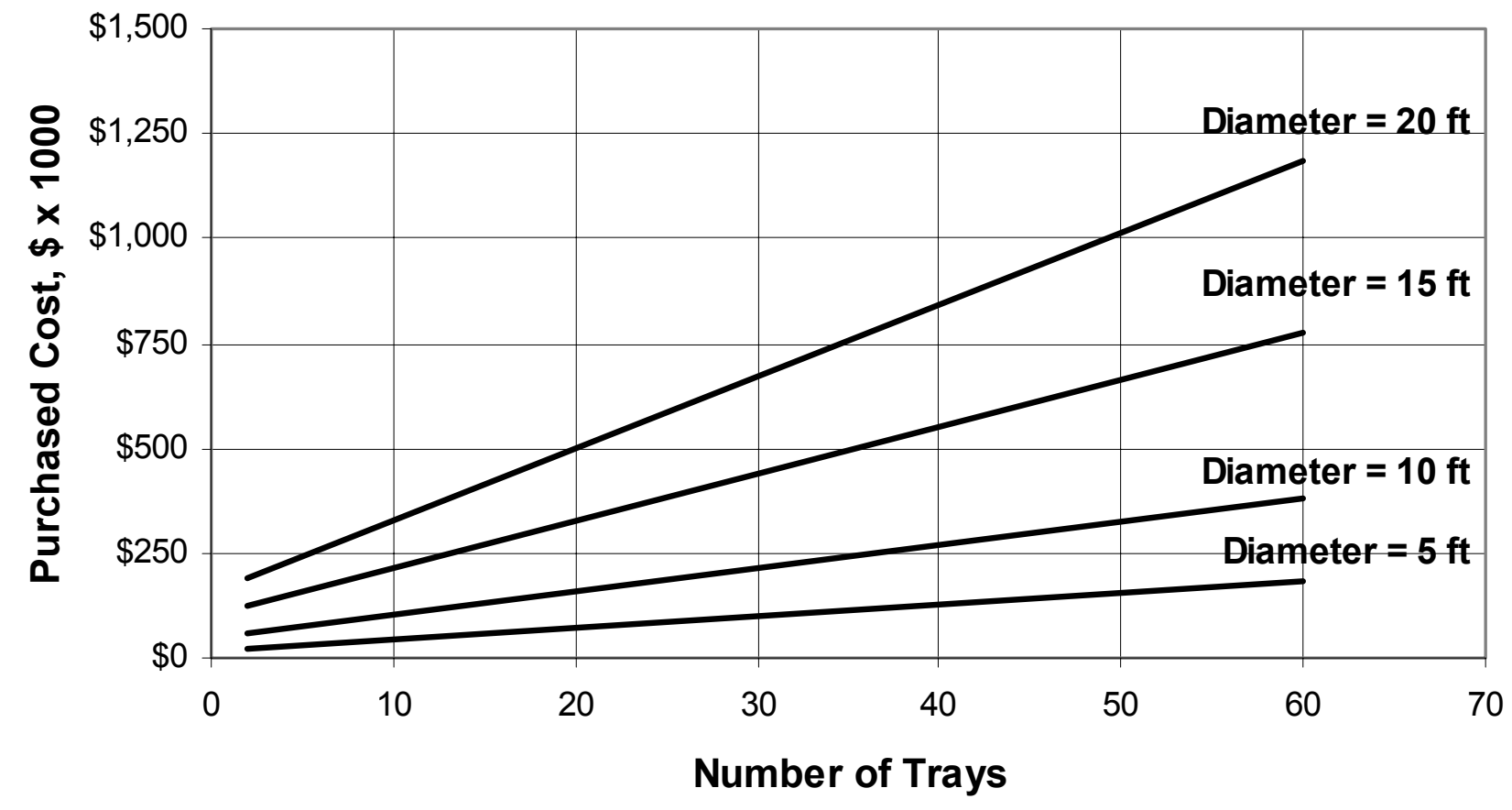




\section{Valve Tray Column - 150 psig}

Description: Pressure/vacuum column includes vessel shell, heads, single base material (lined or clad, nozzles, manholes (one manhole below and above tray stack or packed section and one manhole every tenth tray or 25 feet of packed height), jacket and nozzles for heating or cooling medium, base ring, lugs, skirt or legs; tray clips, tray supports (if designated), distributor piping, plates.

\section{Design Basis:}

$1^{\text {st }}$ Quarter 1998 Dollars

Shell Material: $\quad$ A515

(Carbon Steel Plates for pressure vessels for intermediate and higher temperature service)

Design Temperature: $650{ }^{\circ} \mathrm{F}$

Design Pressure: 150 psig

Height: $\quad 17-133$ feet

Application: Distillation

Tray Type: Valve

Tray Spacing: $\quad 24$ Inches

Tray Material: $\quad$ A285C

(Low and intermediate strength carbon steel plates for pressure vessels.)

Tray Thickness: $\quad 0.19$ Inches

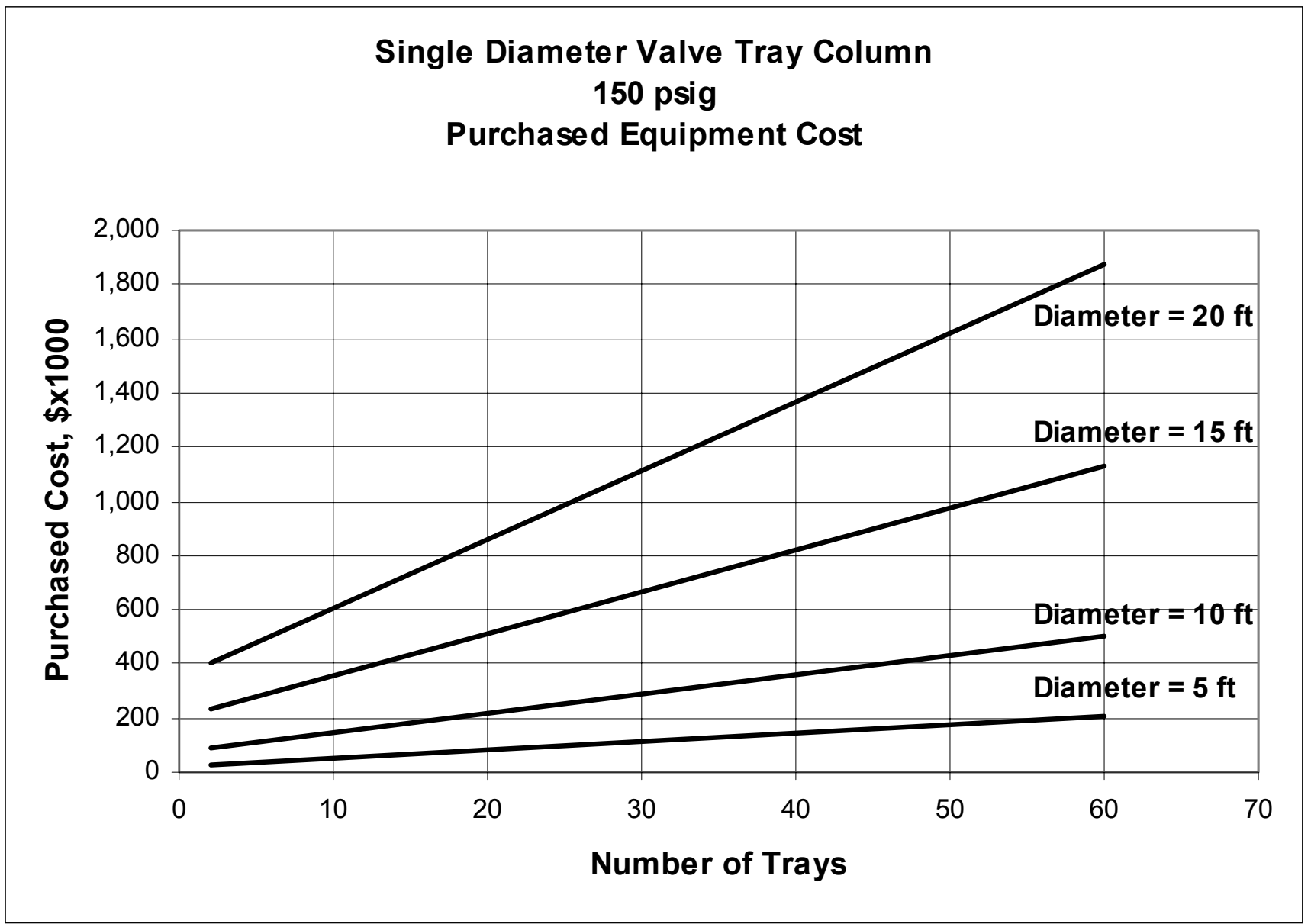




\section{Sieve Tray Column - 15 psig}

Description: Pressure/vacuum column includes vessel shell, heads, single base material (lined or clad, nozzles, manholes (one manhole below and above tray stack or packed section and one manhole every tenth tray or 25 feet of packed height), jacket and nozzles for heating or cooling medium, base ring, lugs, skirt or legs; tray clips, tray supports (if designated), distributor piping, plates.

\section{Design Basis:}

$1^{\text {st }}$ Quarter 1998 Dollars

Shell Material: $\quad$ A515

(Carbon Steel Plates for pressure vessels for intermediate and higher temperature service)

Design Temperature: $650{ }^{\circ} \mathrm{F}$

Design Pressure: 15 psig

Height: 17 - 133 feet

Application: Distillation

Tray Type: Sieve

Tray Spacing: $\quad 24$ Inches

Tray Material: $\quad$ A285C

(Low and intermediate strength carbon steel plates for pressure vessels.)

Tray Thickness: $\quad 0.19$ Inches

\section{Single Diameter Sieve Tray Column 15 psig \\ Purchased Equipment Cost}

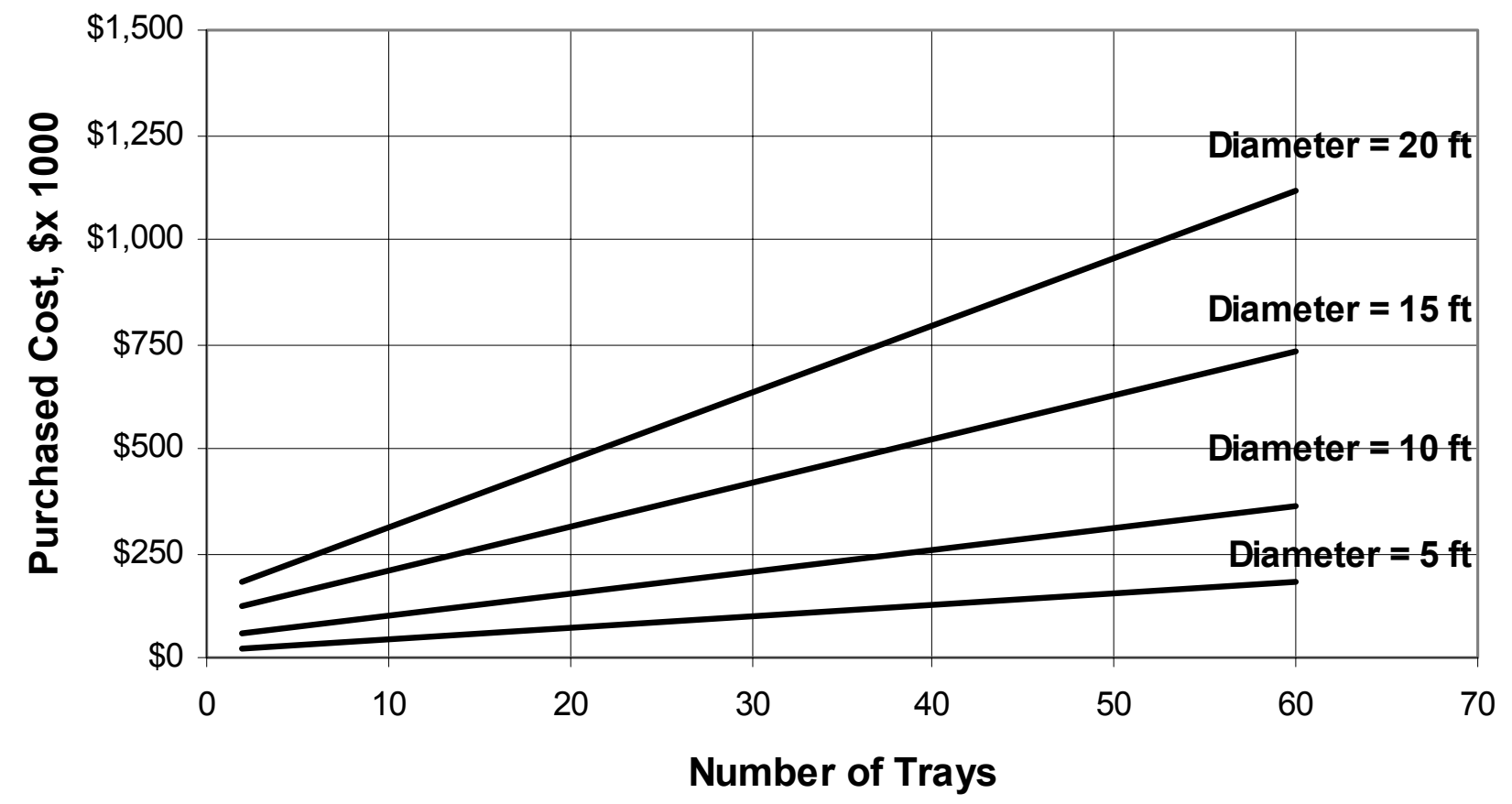




\section{Sieve Tray Column - 150 psig}

Description: Pressure/vacuum column includes vessel shell, heads, single base material (lined or clad, nozzles, manholes (one manhole below and above tray stack or packed section and one manhole every tenth tray or 25 feet of packed height), jacket and nozzles for heating or cooling medium, base ring, lugs, skirt or legs; tray clips, tray supports (if designated), distributor piping, plates.

\section{Design Basis:}

$1^{\text {st }}$ Quarter 1998 Dollars

Shell Material: $\quad$ A515

(Carbon Steel Plates for pressure vessels for intermediate and higher temperature service)

Design Temperature: $650{ }^{\circ} \mathrm{F}$

Design Pressure: $150 \mathrm{psig}$

Height: 17 - 133 feet

Application: Distillation

Tray Type: Sieve

Tray Spacing: $\quad 24$ Inches

Tray Material: $\quad$ A285C

(Low and intermediate strength carbon steel plates for pressure vessels.)

Tray Thickness: $\quad 0.19$ Inches

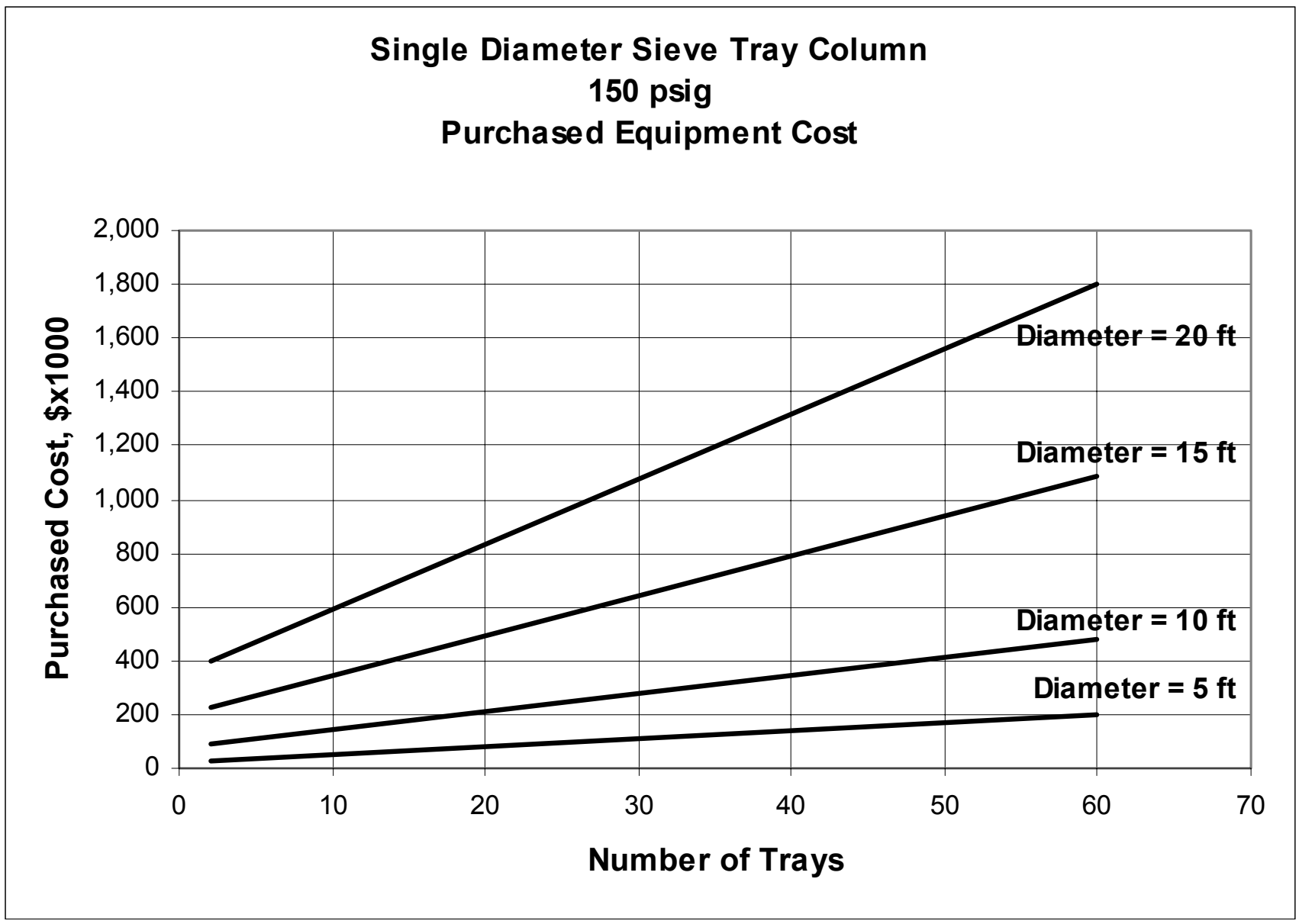




\section{Packed Column - 15 psig}

Description: Pressure/vacuum column includes vessel shell, heads, single base material (lined or clad, nozzles, manholes (one manhole below and above tray stack or packed section and one manhole every tenth tray or 25 feet of packed height), jacket and nozzles for heating or cooling medium, base ring, lugs, skirt or legs; tray clips, tray supports (if designated), distributor piping, plates, packing not included (see Table 1).

\section{Design Basis:}

$1^{\text {st }}$ Quarter 1998 Dollars

Shell Material: $\quad$ A515

(Carbon Steel Plates for pressure vessels for intermediate and higher temperature service)

Design Temperature: $650^{\circ} \mathrm{F}$

Design Pressure: 15 psig

Application: Absorption

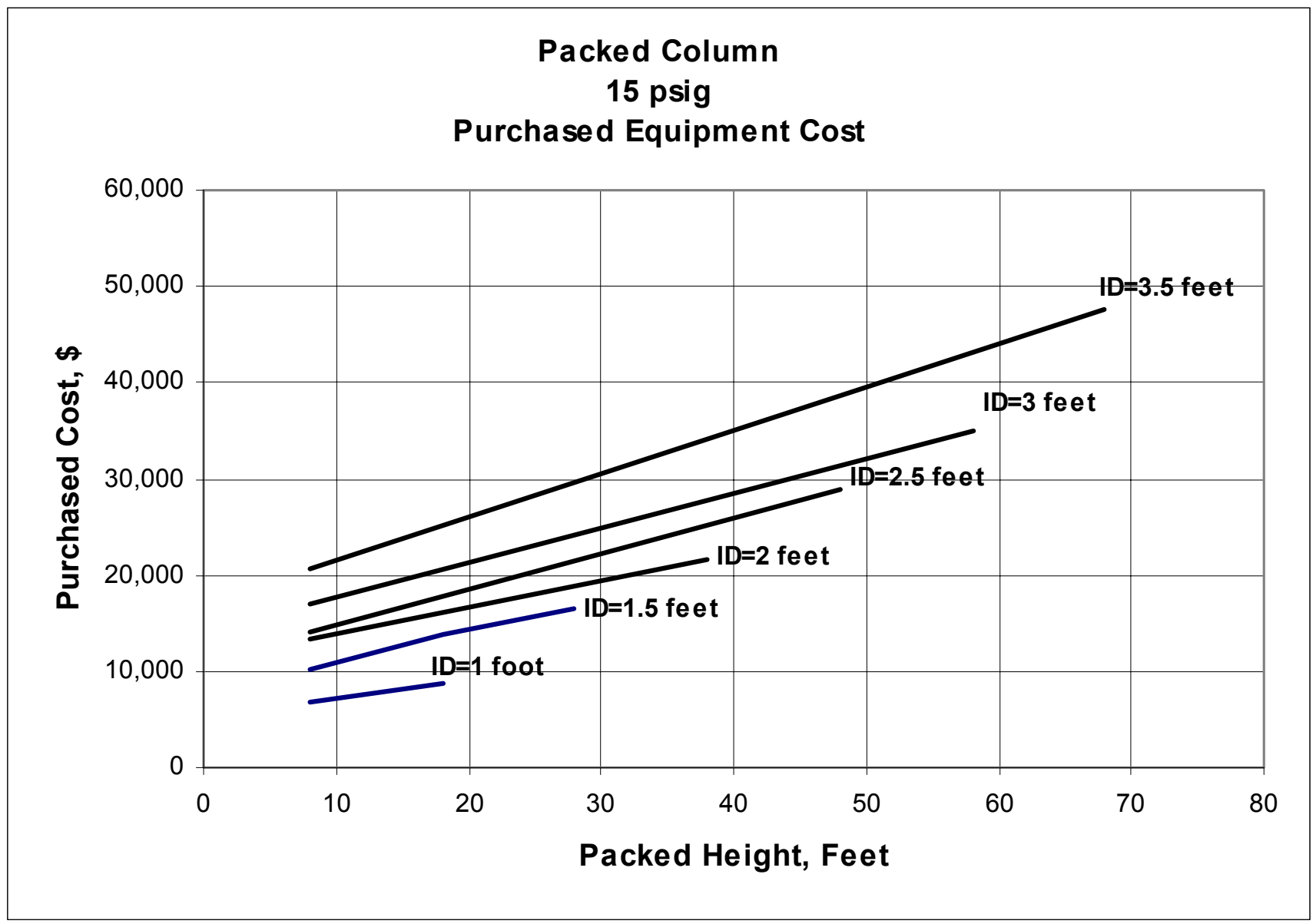




\section{Packed Column - 150 psig}

Description: Pressure/vacuum column includes vessel shell, heads, single base material (lined or clad, nozzles, manholes (one manhole below and above tray stack or packed section and one manhole every tenth tray or 25 feet of packed height), jacket and nozzles for heating or cooling medium, base ring, lugs, skirt or legs; tray clips, tray supports (if designated), distributor piping, plates, packing not included (see Table 1).

\section{Design Basis:}

$1^{\text {st }}$ Quarter 1998 Dollars

Shell Material: $\quad$ A515

(Carbon Steel Plates for pressure vessels for intermediate and higher temperature service)

Design Temperature: $650{ }^{\circ} \mathrm{F}$

Design Pressure: 150 psig

Application: Absorption

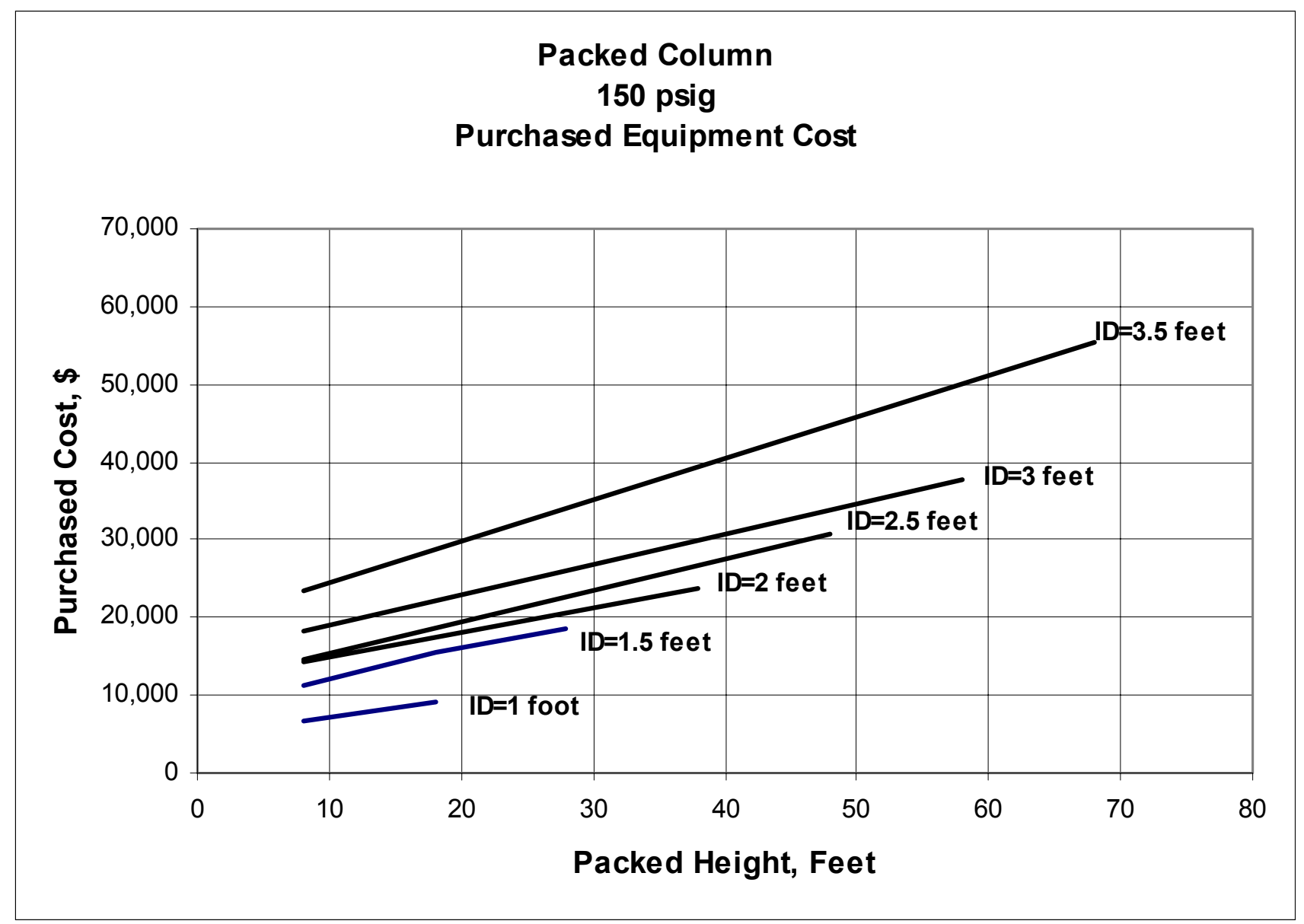


Table 1

Packing Costs

Uninstalled cost, dollar per cubic feet

$1^{\text {st }}$ Quarter 1998 Dollars

\begin{tabular}{|c|c|c|c|c|c|}
\hline Diameter (Inches) & 0.5 & 1.0 & 1.5 & 2.0 & 3.0 \\
\hline \multicolumn{6}{|l|}{ Pall Rings } \\
\hline Polypropylene & 33 & 29 & 21 & 8 & - \\
\hline Stainless Steel & 130 & 118 & 92 & 76 & - \\
\hline \multicolumn{6}{|l|}{ INTALOX Saddles } \\
\hline Ceramic & 31 & 28 & 23 & 21 & - \\
\hline Porcelain & 32 & 29 & 24 & 21 & - \\
\hline \multicolumn{6}{|l|}{ Raschig Rings } \\
\hline Ceramic & 119 & 14 & 12 & 12 & 11 \\
\hline Porcelain & - & 17 & 15 & 12 & 11 \\
\hline Stainless Steel & - & 111 & 94 & 59 & 54 \\
\hline Carbon Steel & - & 37 & 31 & 20 & 18 \\
\hline Activated Carbon & 25 & & & & \\
\hline 13X Molecular Sieve & 61 & & & & \\
\hline Silica Gel & 94 & & & & \\
\hline Calcium Chloride & 11 & & & & \\
\hline
\end{tabular}




\section{Shell and Tube Heat Exchanger}

Description: Shell and tube heat exchanger consists of a bundle of tubes held in a cylindrical shape by plates at either end called tube sheets. The tube bundle placed inside a cylindrical shell. The size of the exchanger is defined as the total outside surface area of the tube bundle. Maximum shell size is 48 Inches.

\section{Design Basis:}

$1^{\text {st }}$ Quarter 1998 Dollars

Type: $\quad$ Floating Head (BES)/ Fixed Head (BEM)

Shell Material: $\quad$ A285C

(Low and intermediate strength carbon steel plates for pressure vessels.)

Shell Temperature: $650^{\circ} \mathrm{F}$

Shell Pressure: $\quad 150 \mathrm{psig}$

Tube Material: $\quad$ A214

(Electric-resistance-welded carbon steel heat exchanger and condenser tubes)

Tube Temperature: $\quad 650^{\circ} \mathrm{F}$

Tube Pressure: $\quad 150 \mathrm{psig}$

Tube Length: $\quad 10-20$ Feet

Tube Diameter: 1 Inch

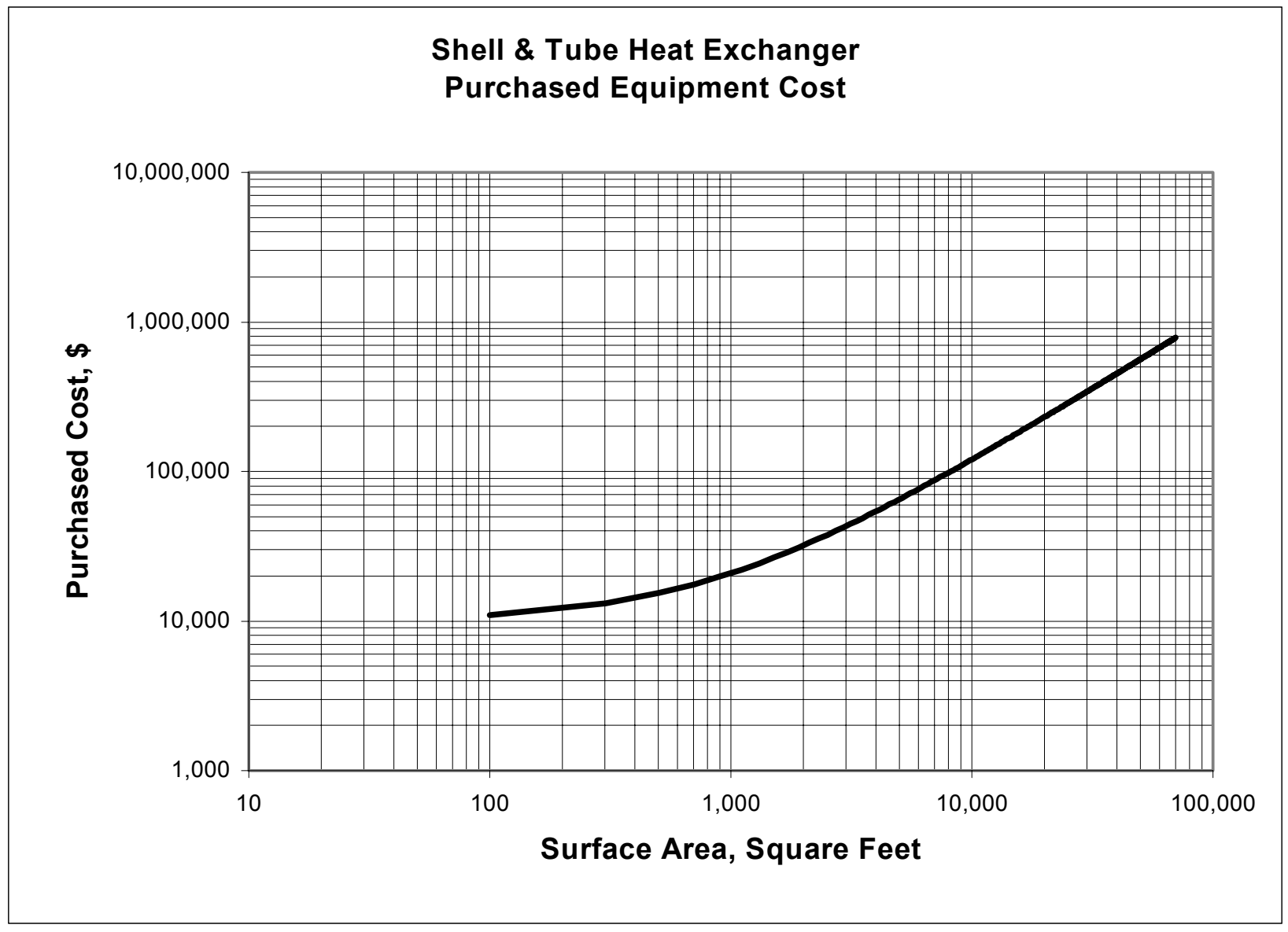




\section{Air Cooler}

Description: Variety of plenum chambers, louver arrangements, fin types (or bare tubes), sizes, materials, free-standing or rack mounted, multiple bays and multiple services within a single bay.

\section{Design Basis:}

$1^{\text {st }}$ Quarter 1998 Dollars

Tube Material: $\quad$ A214

(Electric-resistance-welded carbon steel heat exchanger and condenser tubes)

Tube Length: $\quad 6-60$ Feet

Number of Bays: $\quad 1-3$

Power/ Fan: $\quad 2-25$ Horsepower

Bay Width: $\quad 4-12$ Feet

Design Pressure: 150 psig

Inlet Temperature: $\quad 300^{\circ} \mathrm{F}$

Tube Diameter: 1 Inch

Plenum Type: $\quad$ Transition shaped

Louver Type: $\quad$ Face louvers only

Fin Type: $\quad$ L-footed tension wound Aluminum

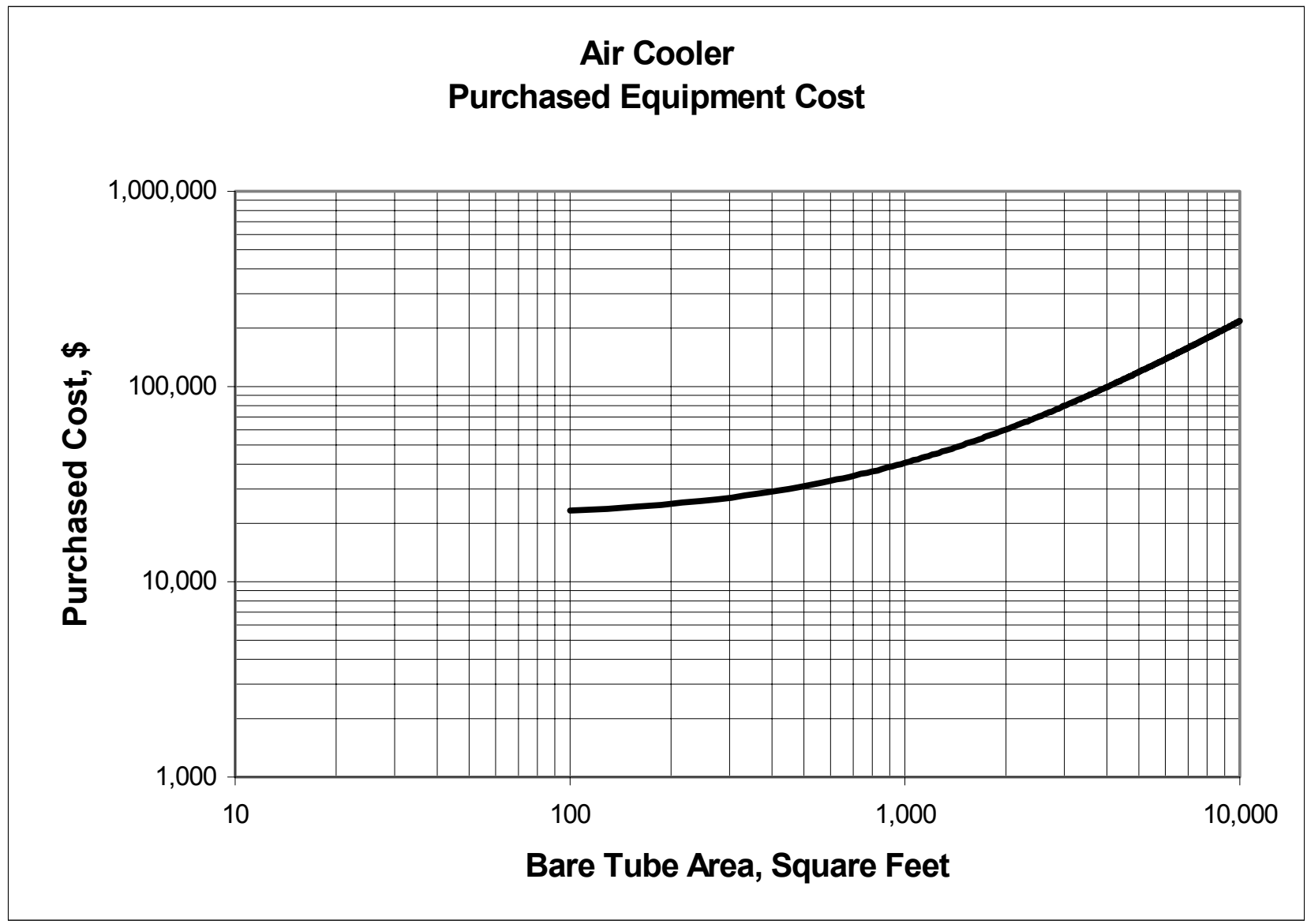




\section{Spiral Plate Heat Exchanger}

\section{Design Basis:}

$1^{\text {st }}$ Quarter 1998 Dollars

Material:

SS304

(High Alloy Steel - Chromium-Nickel stainless steel plate, sheet and strip for fusion-welded unfired pressure vessels)

Tube Pressure: $\quad 150$ psig

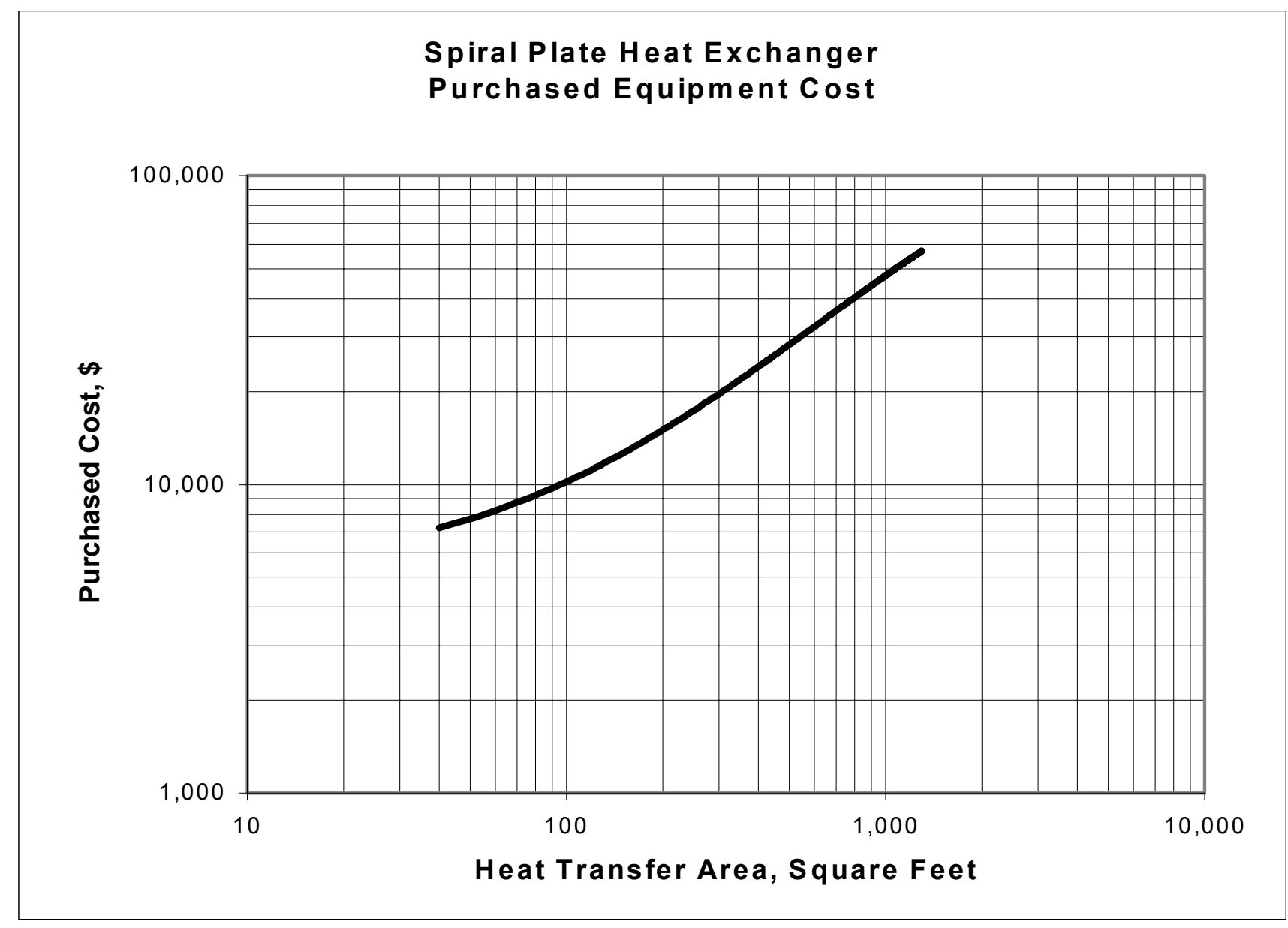




\section{Furnace}

Description: Gas or Oil fired vertical cylindrical type for low heat duty range moderate temperature with long contact time. Walls of the furnace are refractory lined.

\section{Design Basis:}

$1^{\text {st }}$ Quarter 1998 Dollars

\section{Tube Material: $\quad$ A214}

(Electric-resistance-welded carbon steel heat exchanger and condenser tubes)

Design Pressure: 500 psig

Design Temperature: $750{ }^{\circ} \mathrm{F}$

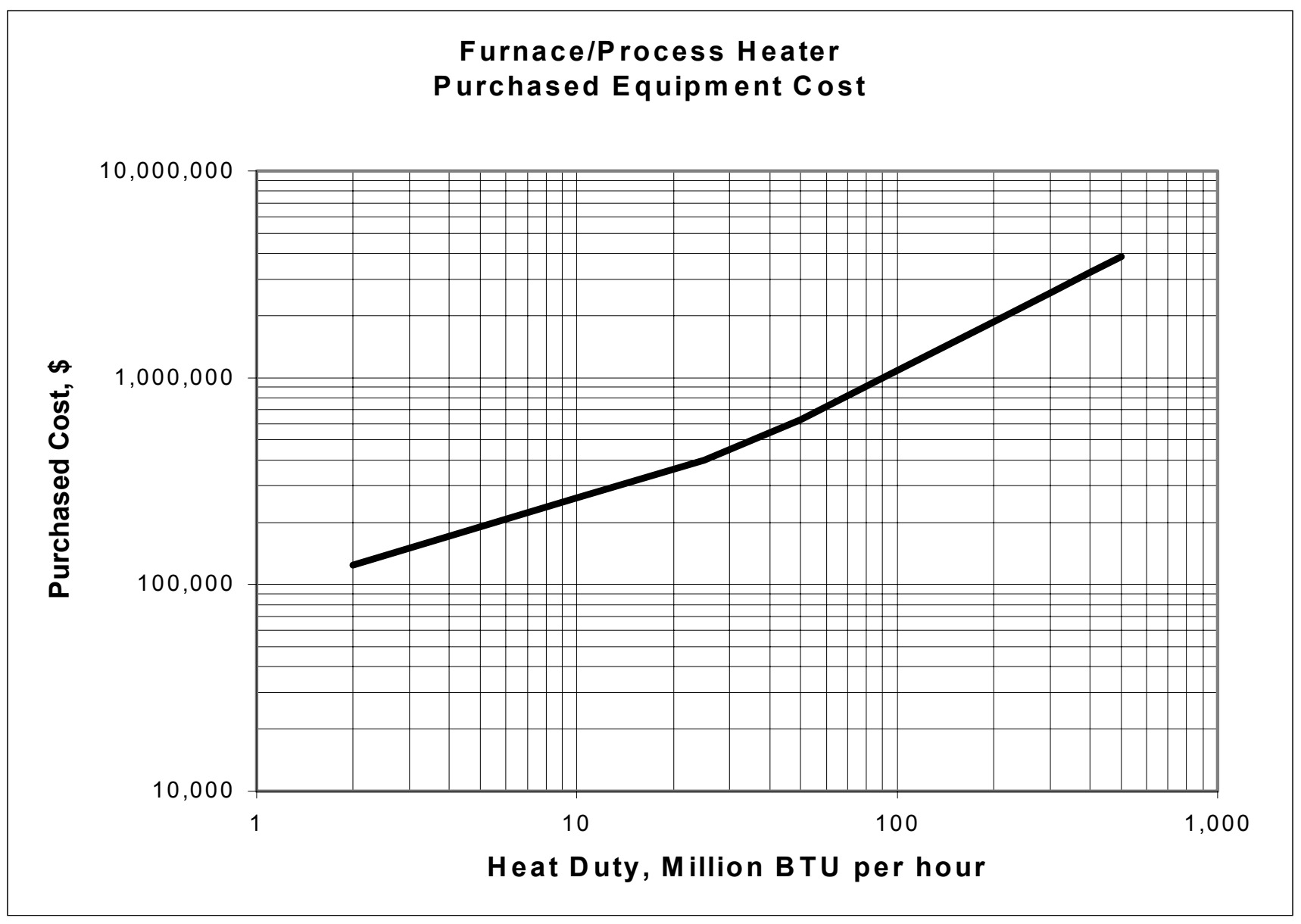




\section{Cooling Tower}

Description: Factory Assembled cooling tower includes fans, drivers and basins

\section{Design Basis:}

$1^{\text {st }}$ Quarter 1998 Dollars

Temperature Range:

$15^{\circ} \mathrm{F}$

Approach Gradient:

$10^{\circ} \mathrm{F}$

Wet Bulb Temperature: $\quad 75^{\circ} \mathrm{F}$

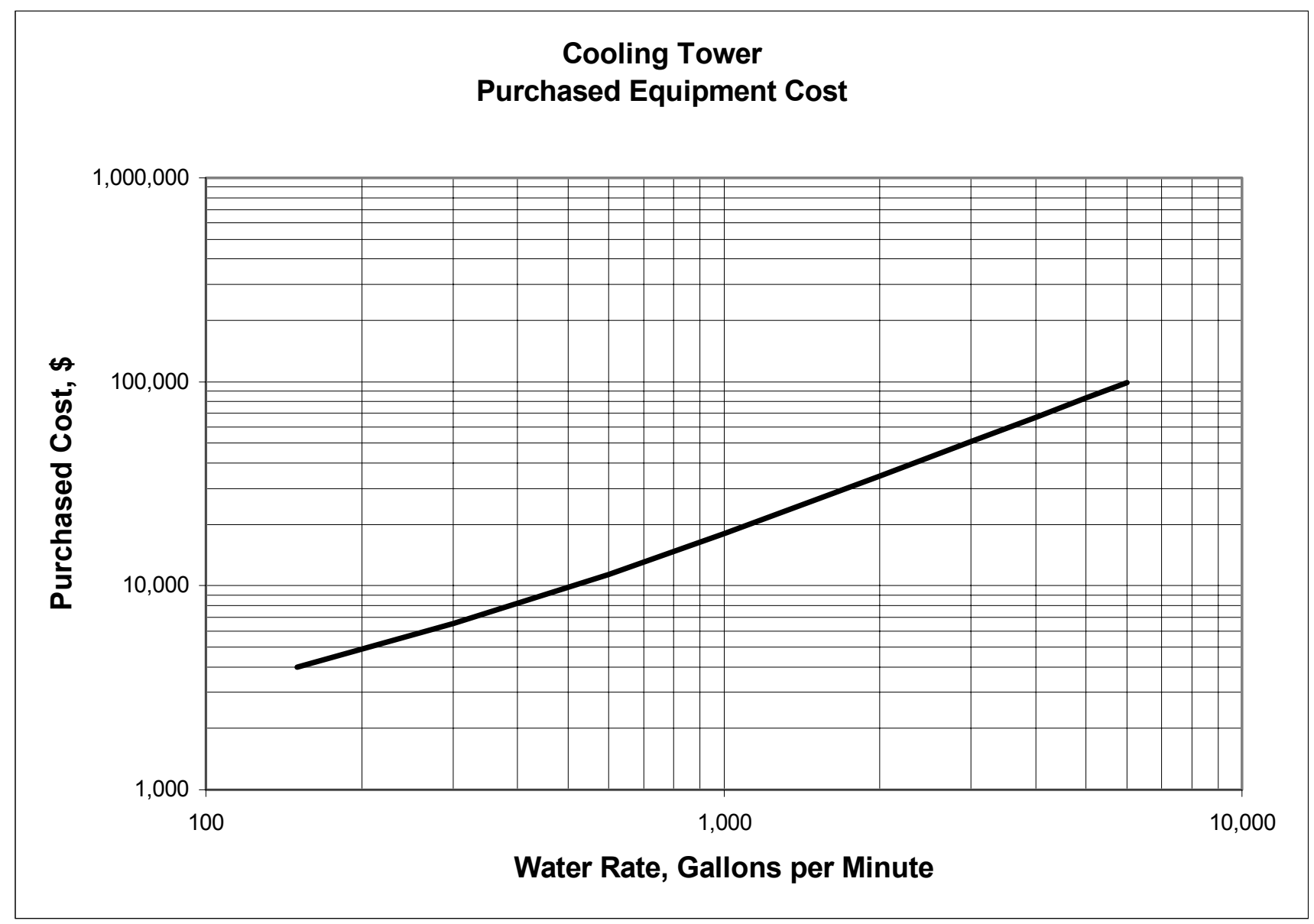




\section{Package Steam Boiler}

Description: Package boiler unit includes forced draft fans, instruments, controls, burners, soot-blowers, feedwater deaerator, chemical injections system, steam drum, mud drum and stack. Shop assembled.

\section{Design Basis:}

$1^{\text {st }}$ Quarter 1998 Dollars

Material: A285C

(Low and intermediate strength carbon steel plates for pressure vessels.)

Pressure: $250 \mathrm{psig}$

Superheat: $\quad 100^{\circ} \mathrm{F}$

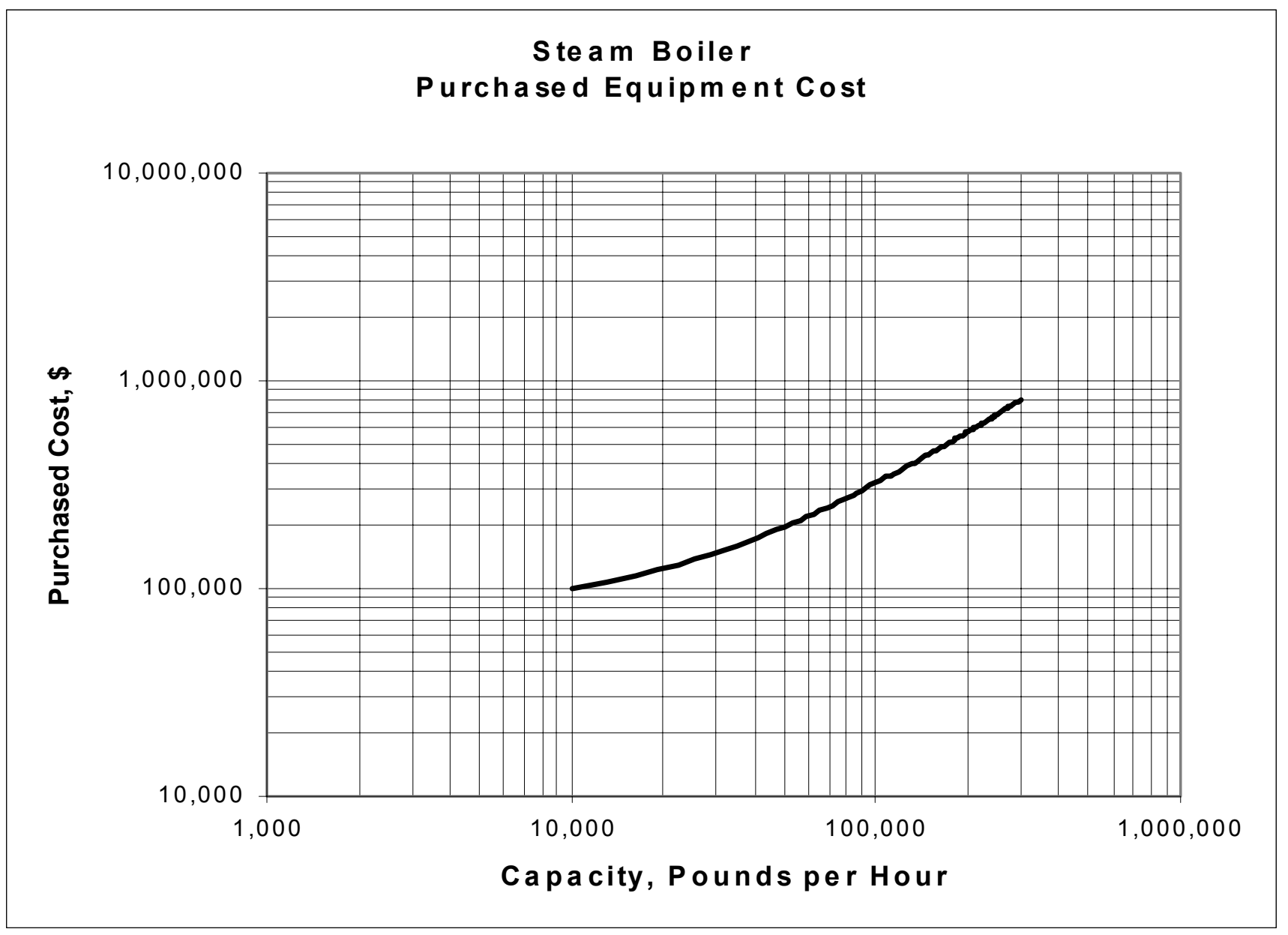




\section{Evaporators}

Description: Standard vertical tube evaporator and standard horizontal tube evaporator.

\section{Design Basis:}

$1^{\text {st }}$ Quarter 1998 Dollars

Material:

A285C

(Low and intermediate strength carbon steel plates for pressure vessels.)

Tube Material: $\quad$ Carbon Steel

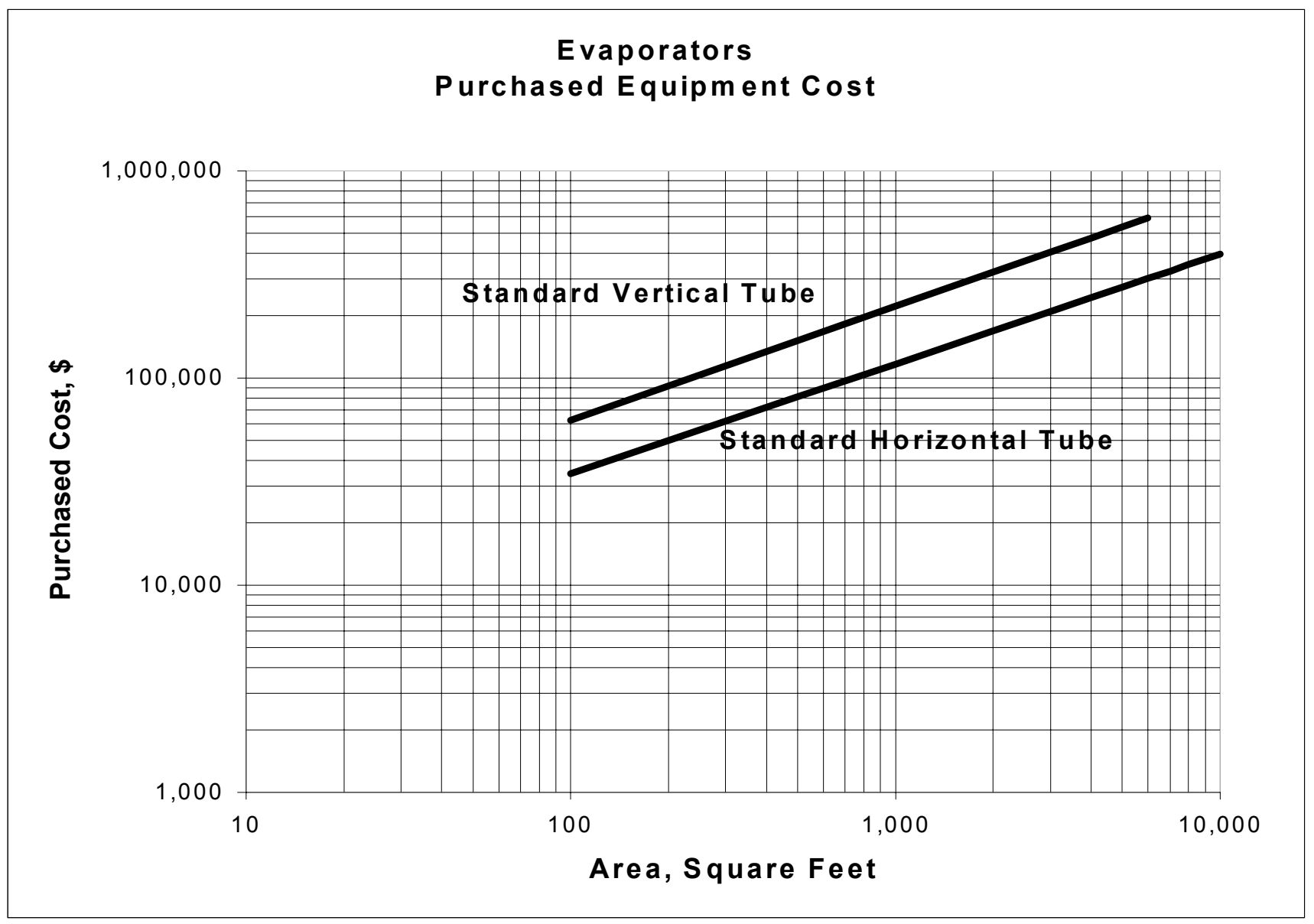




\section{Crushers}

Description: All crushers include motor and drive unit.

Gyratory: Primary crushing of hard and medium hard materials.

Rotary: For course, soft materials.

Ring Granulator: For primary and secondary crushing of bituminous and subbituminous coals, lignite, gypsum and some medium hard minerals.

\section{Design Basis:}

$1^{\text {st }}$ Quarter 1998 Dollars

Material: $\quad$ A285C

(Low and intermediate strength carbon steel plates for pressure vessels.)

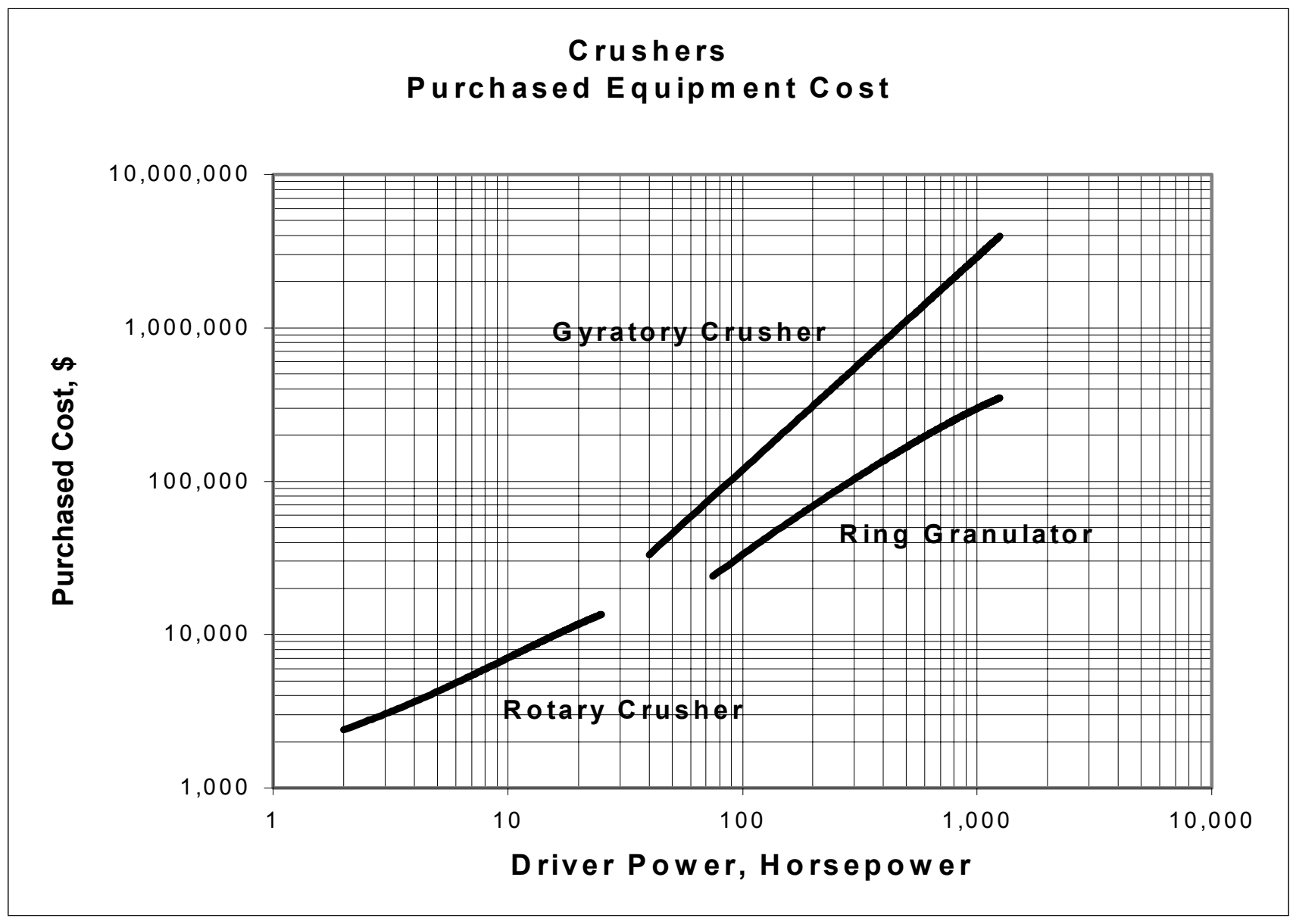




\section{Mills}

Description: All units include mill, bearings, gears, lube system and vendor-supplied instruments. Ball mill includes initial ball charge.

\section{Design Basis:}

$1^{\text {st }}$ Quarter 1998 Dollars

Material:

$\mathrm{A} 285 \mathrm{C}$

(Low and intermediate strength carbon steel plates for pressure vessels.)

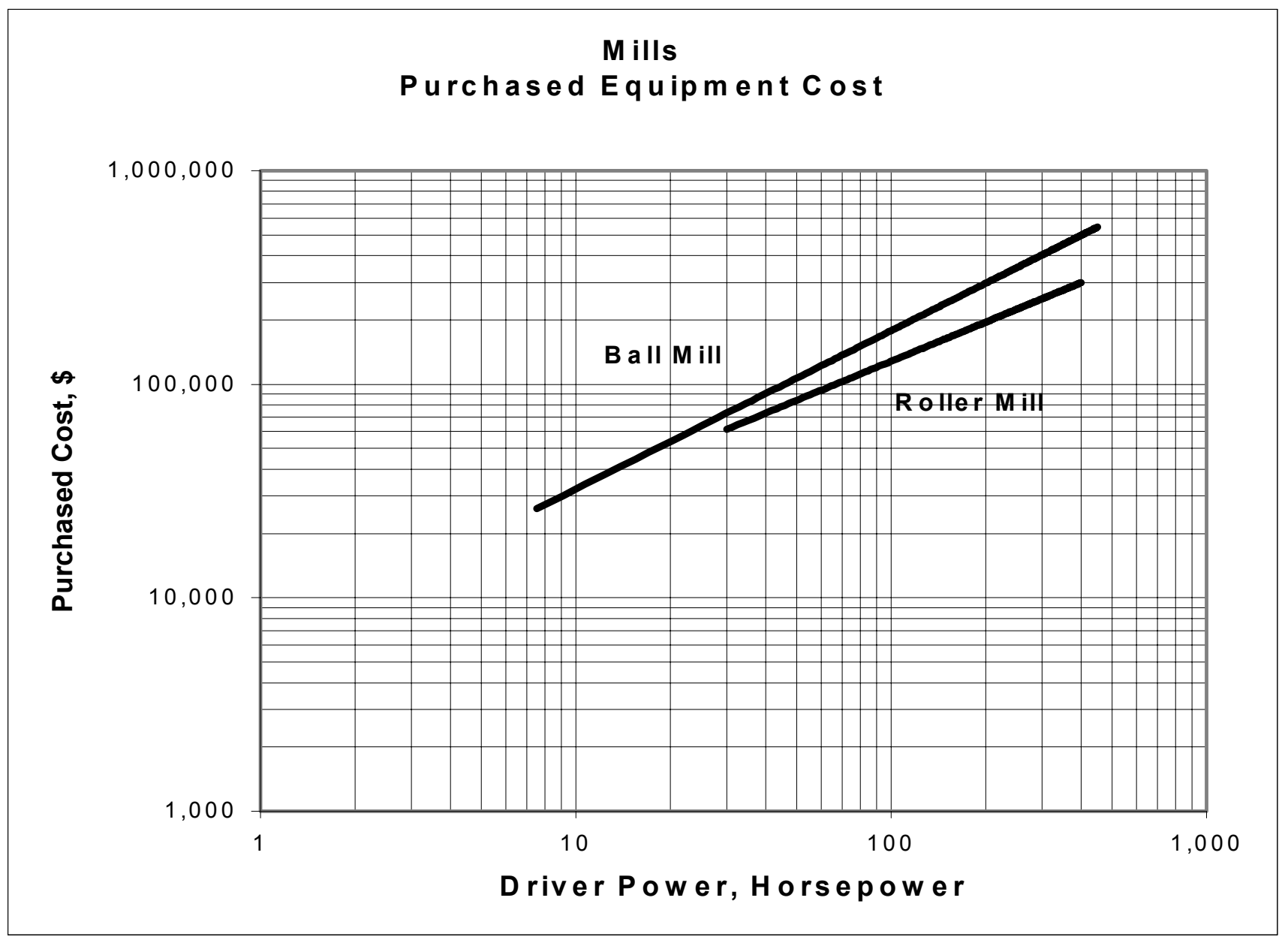




\section{Dryers}

\section{Description:}

Atmospheric tray batch dryer includes solid materials.

Rotary and Drum dryers include motor and drive unit.

\section{Design Basis:}

$1^{\text {st }}$ Quarter 1998 Dollars

Material:

(Low and intermediate strength carbon steel plates for pressure vessels.)

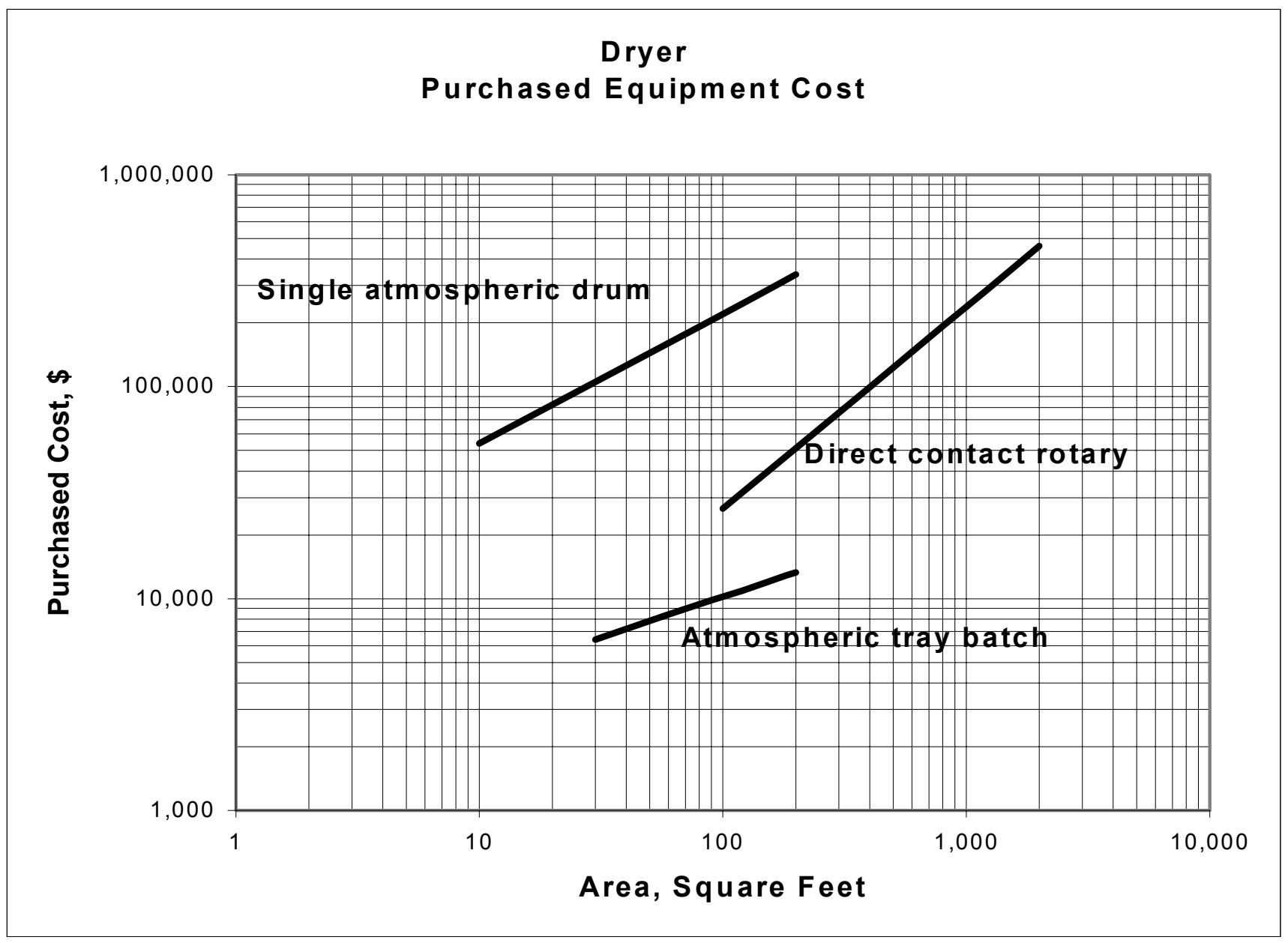




\section{Centrifuges}

Description: Centrifuges include motor and drive unit.

Reciprocating Conveyor with continuous filtering centrifuge for free-draining granular solids, horizontal bowl, removal by reciprocating piston.

Continuous Filtration Vibratory Centrifuge with solids removal by vibratory screen for dewatering of course solids.

\section{Design Basis:}

$1^{\text {st }}$ Quarter 1998 Dollars

Material: $\quad$ A285C

(Low and intermediate strength carbon steel plates for pressure vessels.)

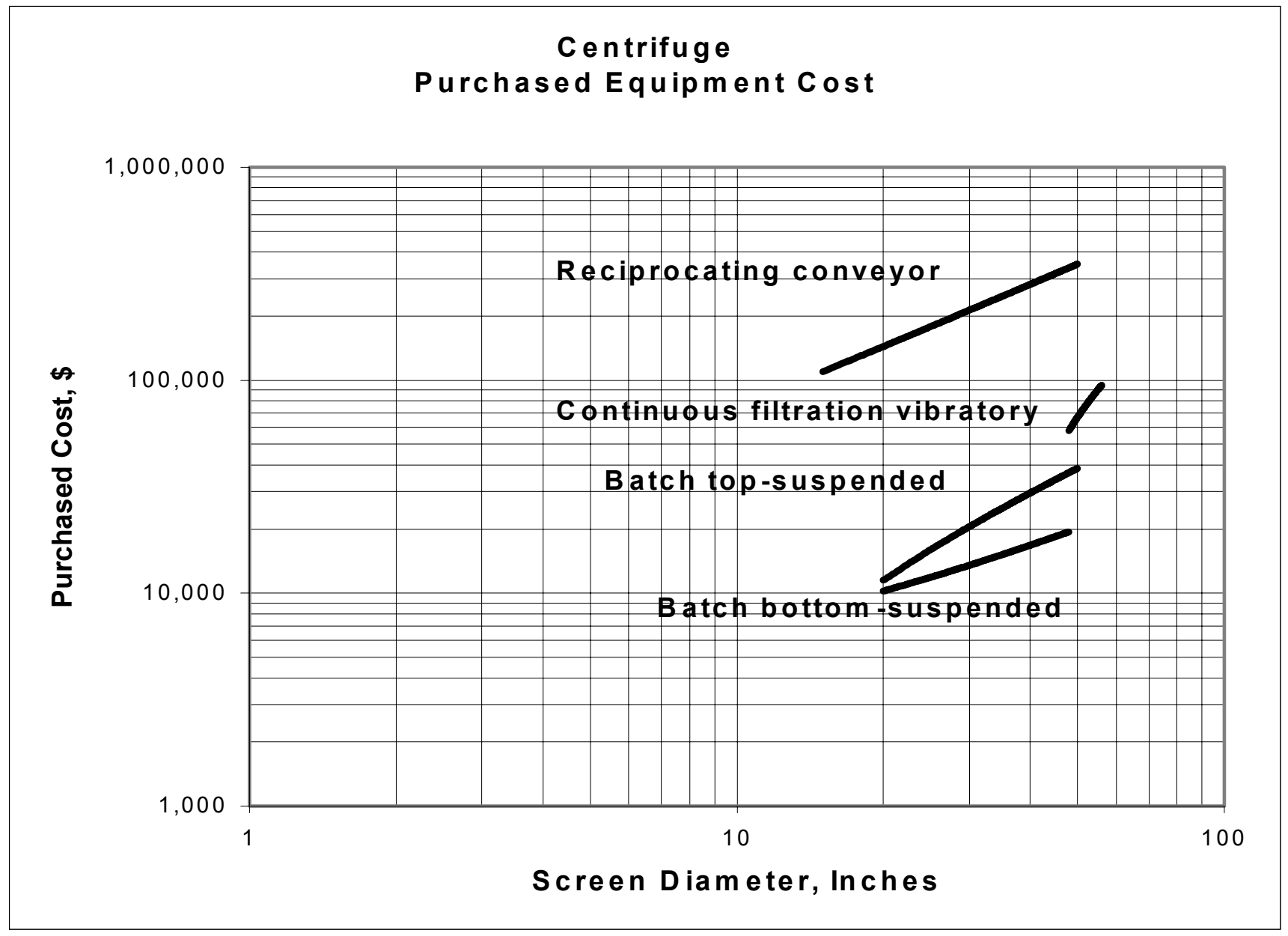




\section{Filters}

\section{Description:}

Cartridge Filter consists of a tank containing one or more disposable cartridges.

Contains 5-micron cotton filter.

Drum Filter is a vacuum type, multi compartment cylinder shell with internal filtrate piping with polypropylene filter cloth, feed box with inlet and drain nozzles, suction valve, discharge trough, driver consisting of rotor, drive motor base plate, worm, gear reducer and two pillow block bearing with supports.

Defaults for Drum Filter

medium filtration rate,

0.5 tons per day/ square feet solids handling rate,

$20 \%$ consistency (percent of solids in feed stream).

Tubular Fabric Filters are a bank of three without automatic cleaning option.

Plate and Frame Filter default material is rubber-lined carbon steel.

\section{Design Basis:}

$1^{\text {st }}$ Quarter 1998 Dollars

Material: A285C

(Low and intermediate strength carbon steel plates for pressure vessels.)

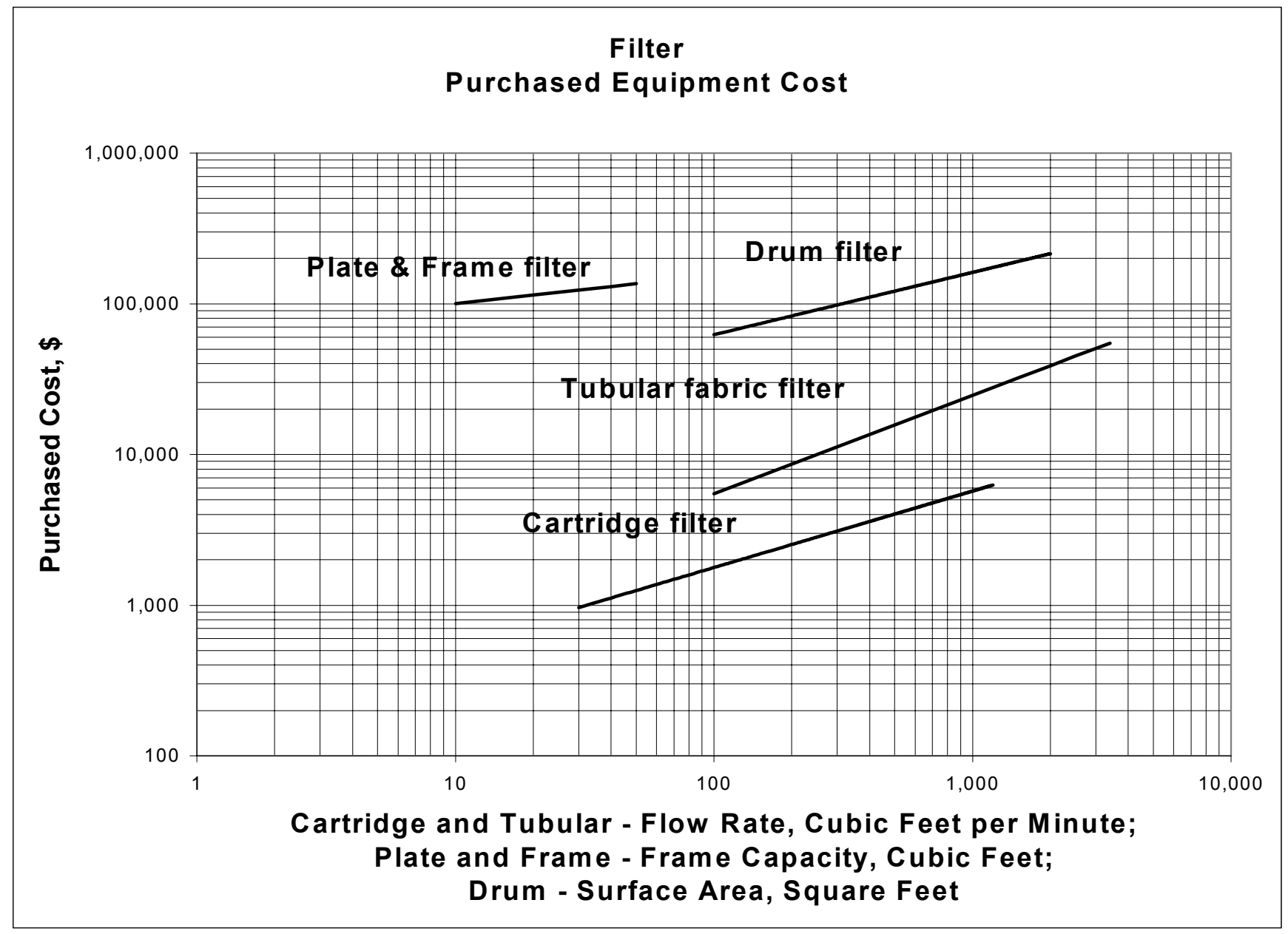




\section{Agitator}

Description: Fixed propeller mixer with motor and gear drive. Includes motor, gear drive, shaft and impeller.

\section{Design Basis:}

$1^{\text {st }}$ Quarter 1998 Dollars

Material:

$\mathrm{A} 285 \mathrm{C}$

(Low and intermediate strength carbon steel plates for pressure vessels.)

Speed:

1800 RPM

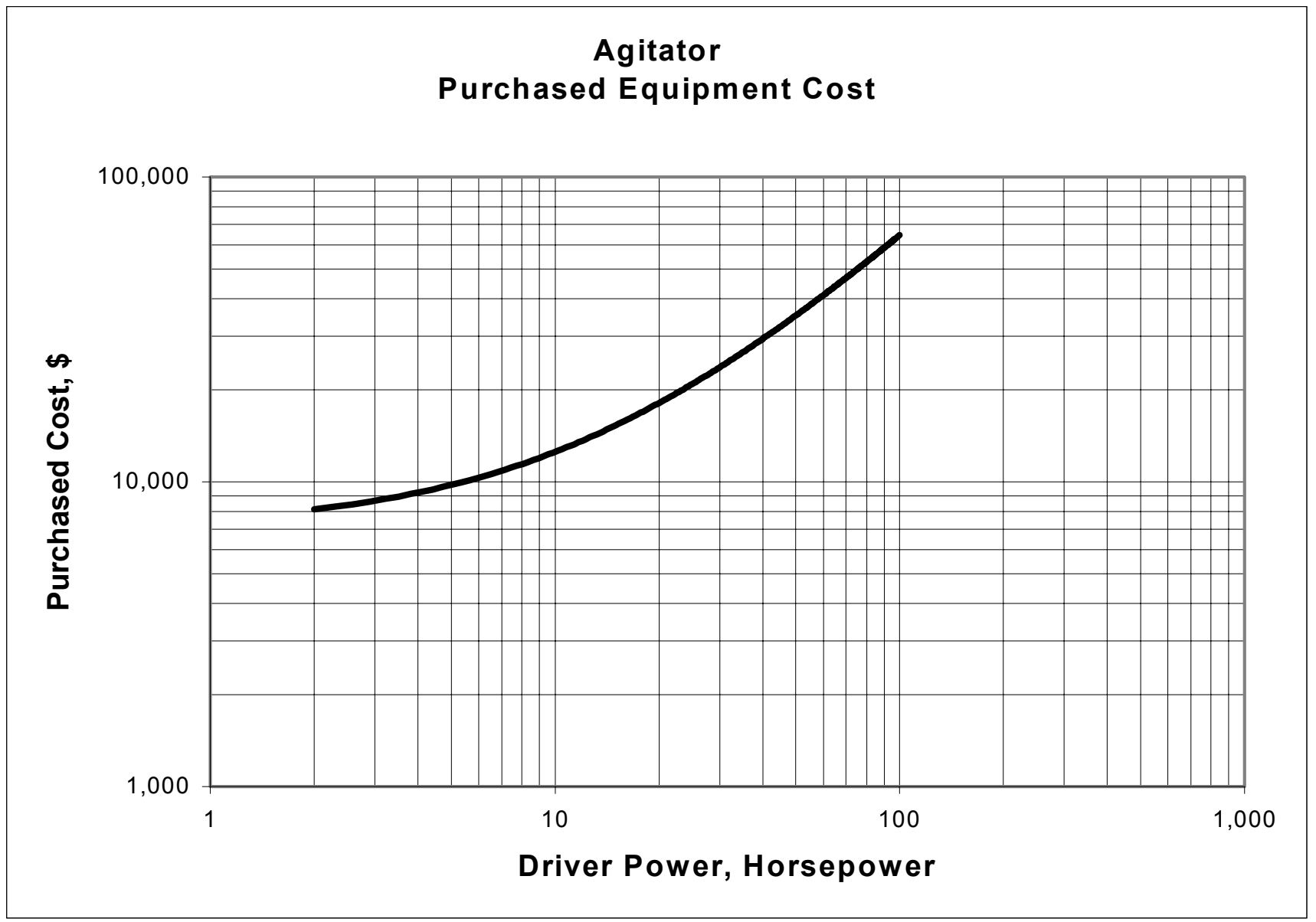




\section{Rotary Pump}

Description: Rotary (sliding vanes) pump includes motor driver.

\section{Design Basis:}

$1^{\text {st }}$ Quarter 1998 Dollars

Material:

Cast Iron

Temperature:

$68^{\circ} \mathrm{F}$

Power:

25 - 20 Horsepower

Speed:

1800 RPM

Liquid Specific Gravity: 1

Efficiency:

$82 \%$

\section{Rotary Pump \\ Purchased Equipment Cost}

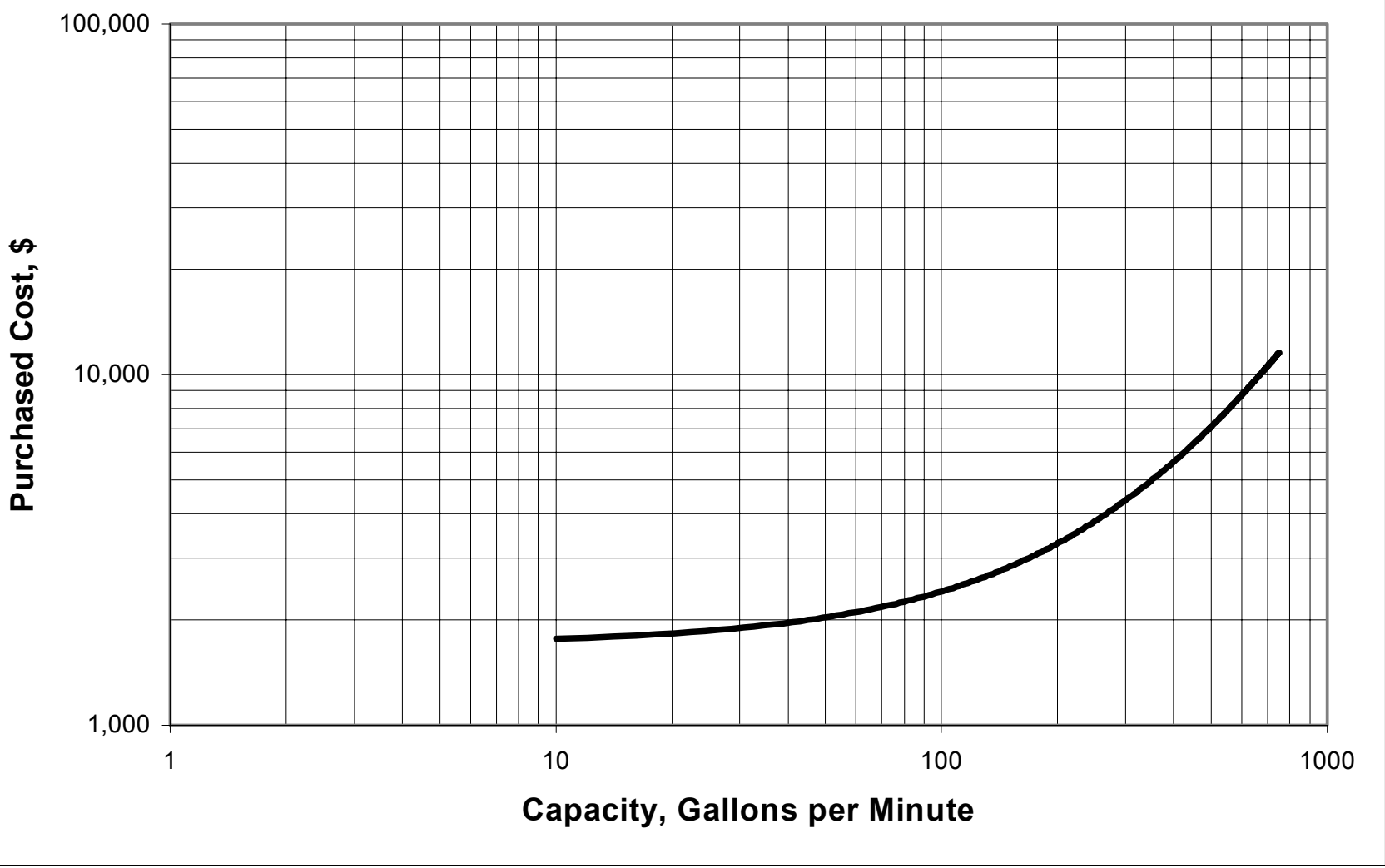




\section{Inline Pump}

Description: General service in-line pump includes pump and motor driver.

\section{Design Basis:}

$1^{\text {st }}$ Quarter 1998 Dollars

Material:

Carbon Steel

Temperature:

$120^{\circ} \mathrm{F}$

Speed:

1800 RPM

Liquid Specific Gravity:1

Efficiency:

$<50 \mathrm{GPM}=60 \%$

$50-199 \mathrm{GPM}=65 \%$

$100-500 \mathrm{GPM}=75 \%$

$>500 \mathrm{GPM}=82 \%$

Driver Type:

Standard motor

Seal Type:

Single mechanical seal

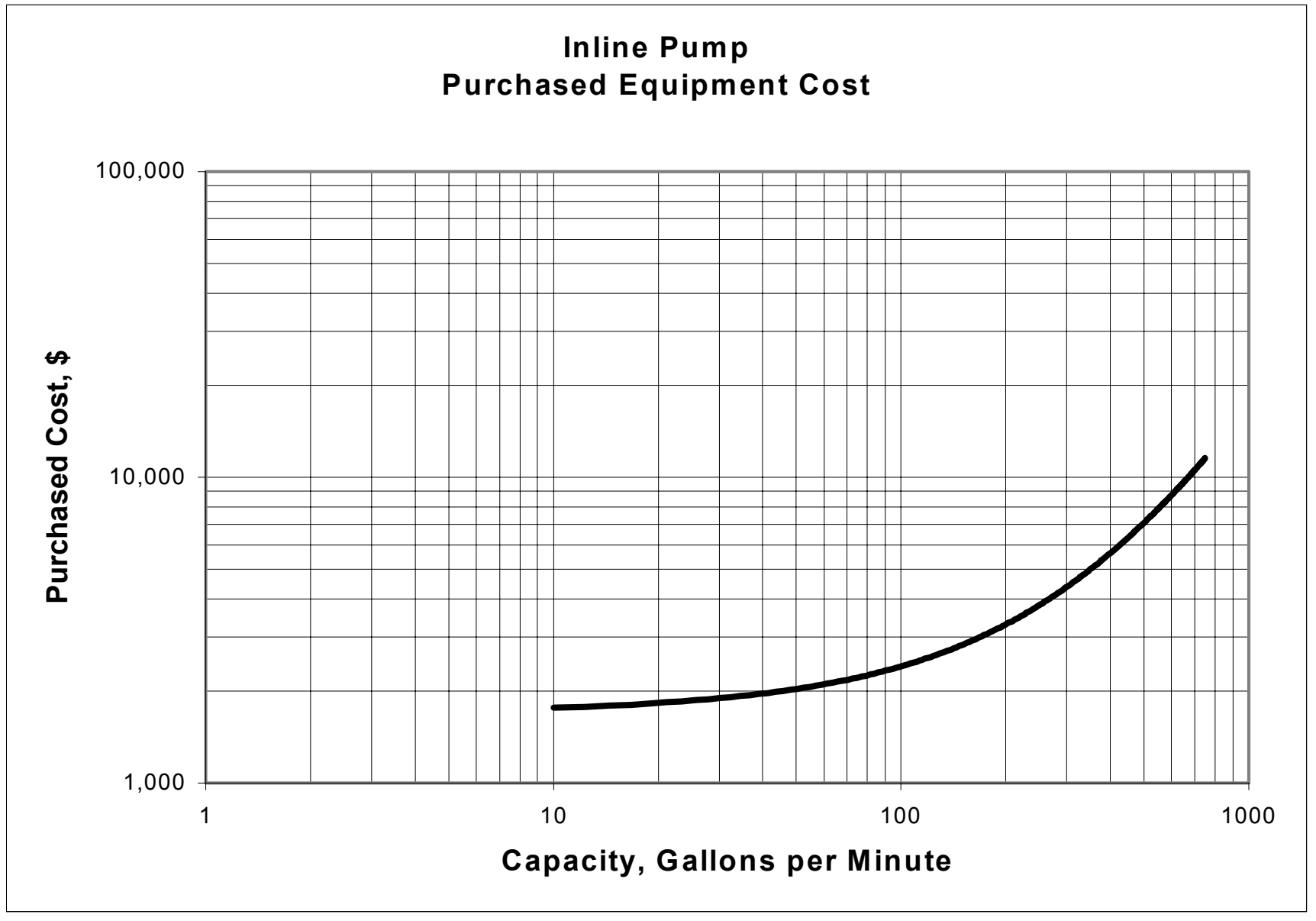




\section{Centrifugal Pump}

Description: Single and multistage centrifugal pumps for process or general service when flow/head conditions exceed general service. Split casing not a cartridge or barrel. Includes standard motor driver.

\section{Design Basis:}

$1^{\text {st }}$ Quarter 1998 Dollars

Material: $\quad$ Carbon Steel

Design Temperature: $120^{\circ} \mathrm{F}$

Design Pressure: 150 psig

Liquid Specific Gravity:1

Efficiency:

$$
\begin{aligned}
& <50 \mathrm{GPM}=60 \% \\
& 50-199 \mathrm{GPM}=65 \% \\
& 100-500 \mathrm{GPM}=75 \% \\
& >500 \mathrm{GPM}=82 \%
\end{aligned}
$$

Driver Type: $\quad$ Standard motor

Seal Type: $\quad$ Single mechanical seal

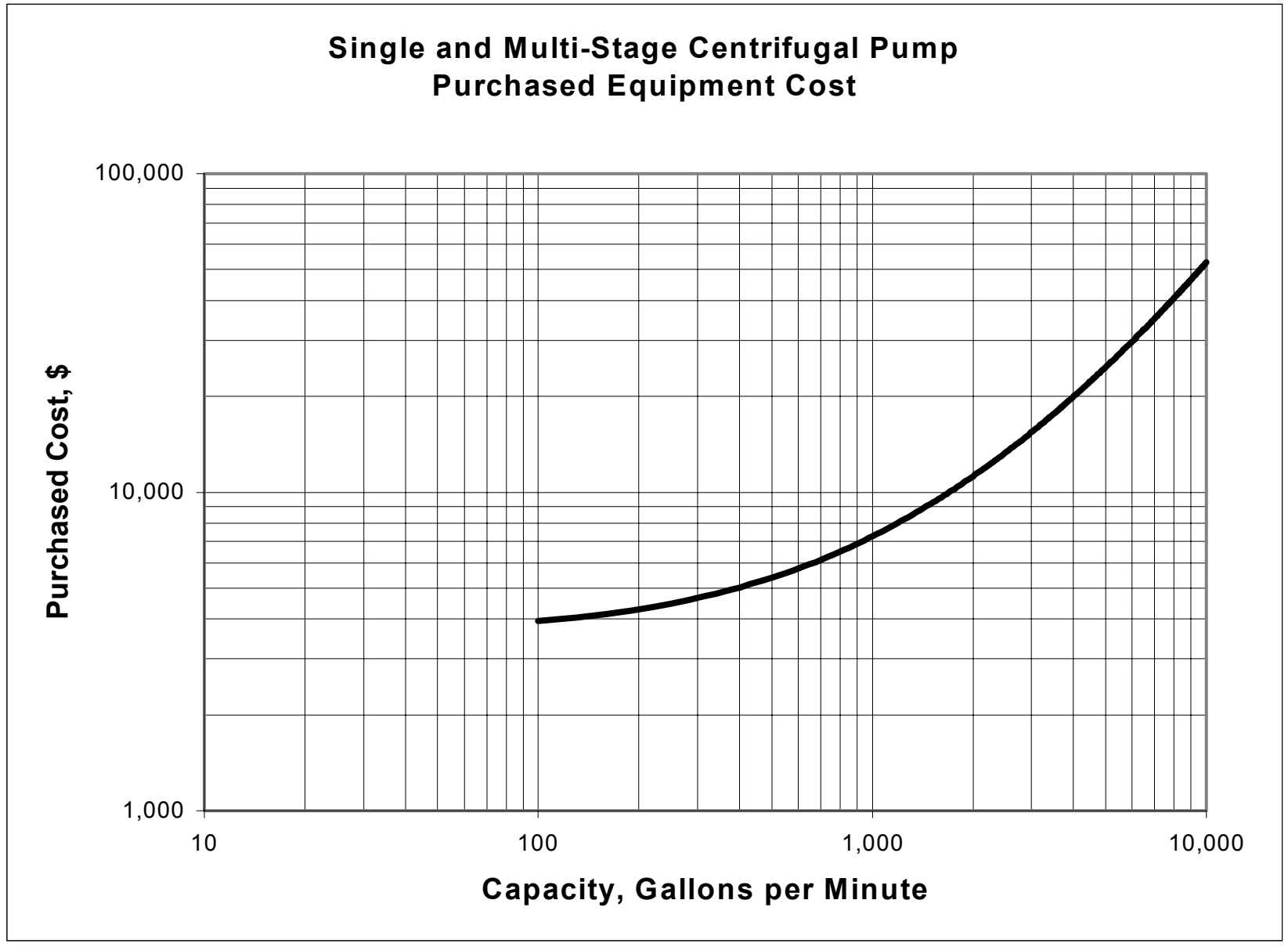




\section{Reciprocating Pump}

Description: Reciprocating duplex with steam driver. Triplex (plunger) with pumpmotor driver.

\section{Design Basis:}

$1^{\text {st }}$ Quarter 1998 Dollars

Material: Carbon Steel

Design Temperature: $68^{\circ} \mathrm{F}$

Liquid Specific Gravity: 1

Efficiency: $\quad 82 \%$

\section{Reciprocating Pump Purchased Equipment Cost}

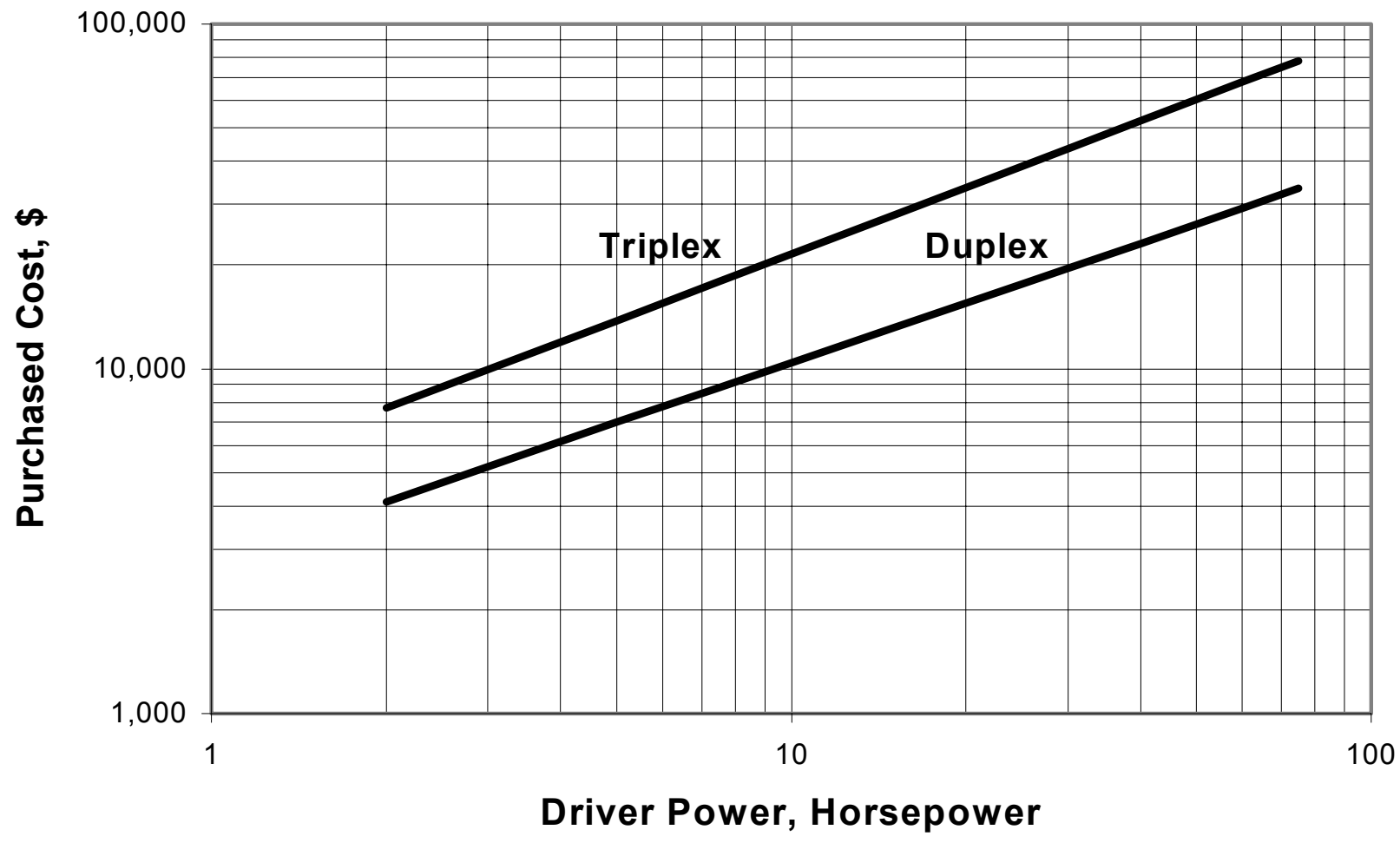




\section{Vacuum Pump}

Description: Mechanical oil-sealed vacuum pump includes pump, motor and drive unit.

\section{Design Basis:}

$1^{\text {st }}$ Quarter 1998 Dollars

Material:

Carbon Steel

First Stage:

0.01 MM HG (Mercury)

Second Stage:

0.0003 MM HG (Mercury)

\section{Vacuum Pump Purchased Equipment Cost}

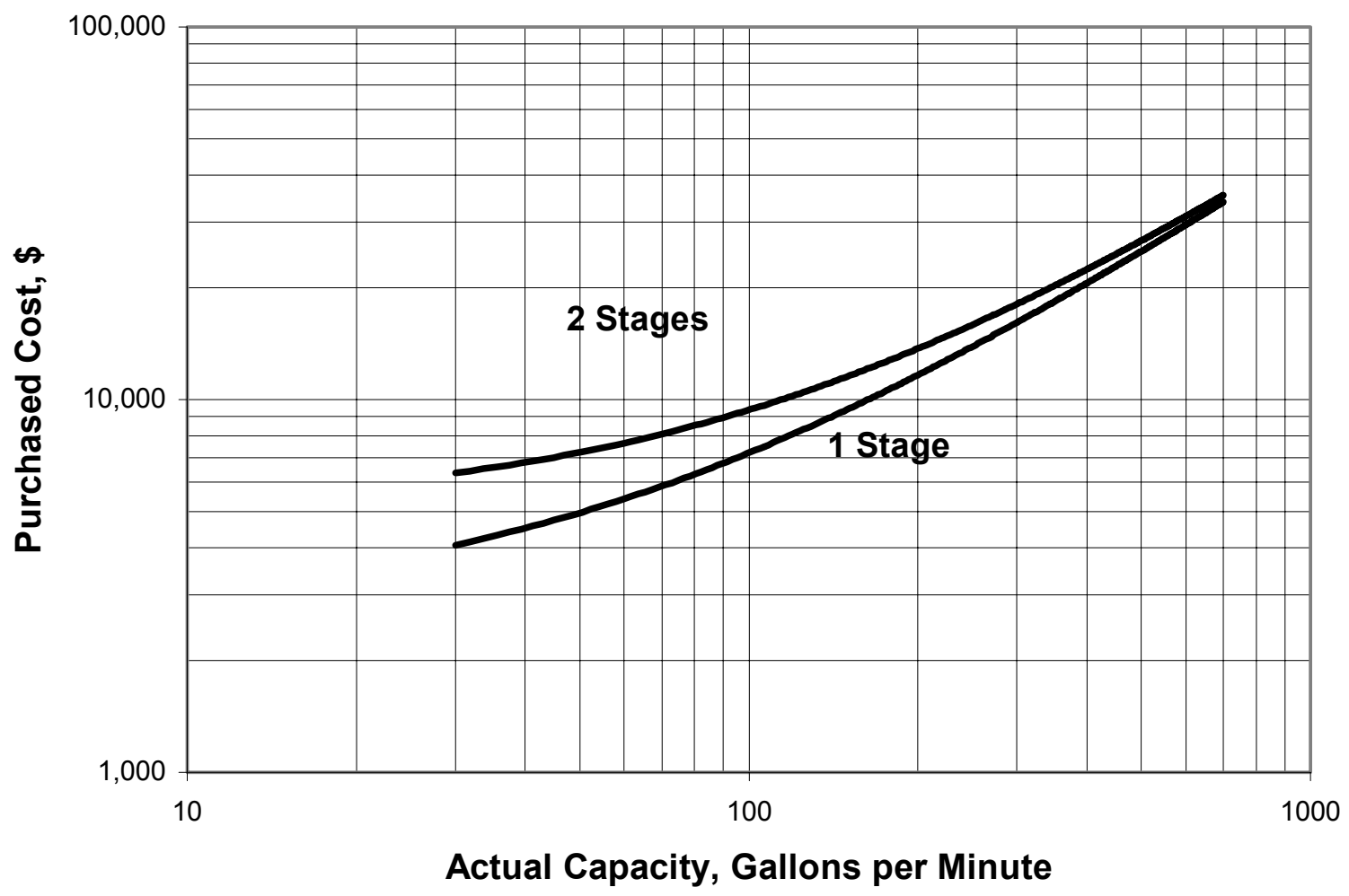




\section{Reciprocating Compressor}

Description: Reciprocating compressor with gear reducer, couplings, guards, base plate, compressor unit, fittings, interconnecting piping, vendor-supplied instruments, lube/seal system. Does not include intercoolers or aftercoolers and interstage knock-out drums.

\section{Design Basis:}

$1^{\text {st }}$ Quarter 1998 Dollars

Material: Carbon Steel

Inlet Temperature: $\quad 68^{\circ} \mathrm{F}$

Inlet Pressures: $\quad 14.7 / 14.7 / 165$ psia

Pressure Ratios: $\quad$ 4:1/30:1/30:1

Molecular Weight: 30

Specific Heat Ratio: 1.22

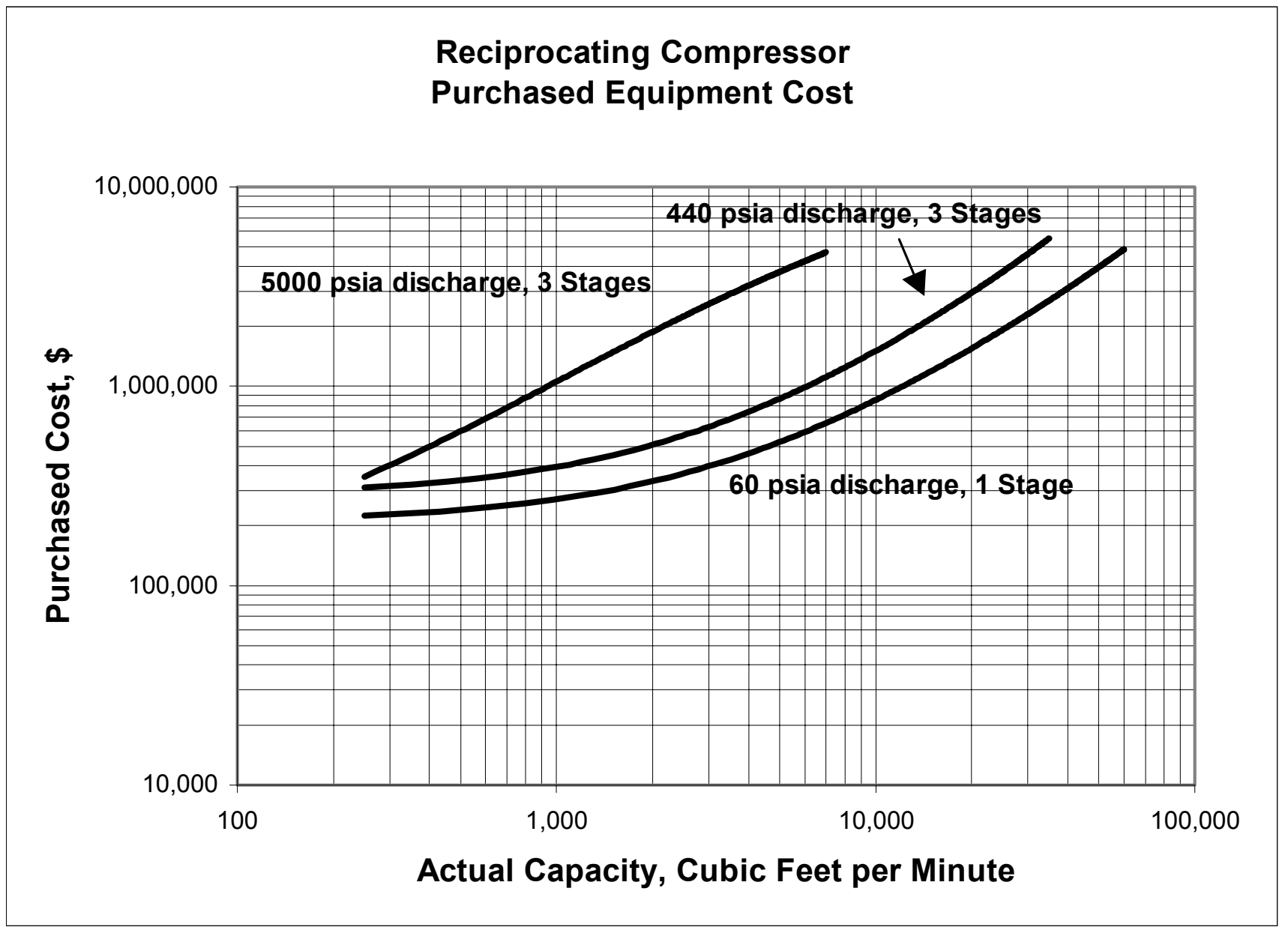




\section{Centrifugal Compressor}

Description: Axial (inline) centrifugal gas compressor with motor driver. Excludes intercoolers and knock-out drums.

\section{Design Basis:}

$1^{\text {st }}$ Quarter 1998 Dollars

Material: $\quad$ Carbon Steel

Inlet Temperature: $\quad 68^{\circ} \mathrm{F}$

Inlet Pressures: $\quad$ 14.7/ $14.7 / 190$ psia

Pressure Ratios: $\quad 3: 1 / 10: 1 / 10: 1$

Molecular Weight: 29

Specific Heat Ratio: 1.4

\section{Centrifugal Compressor \\ Purchased Equipment Cost}

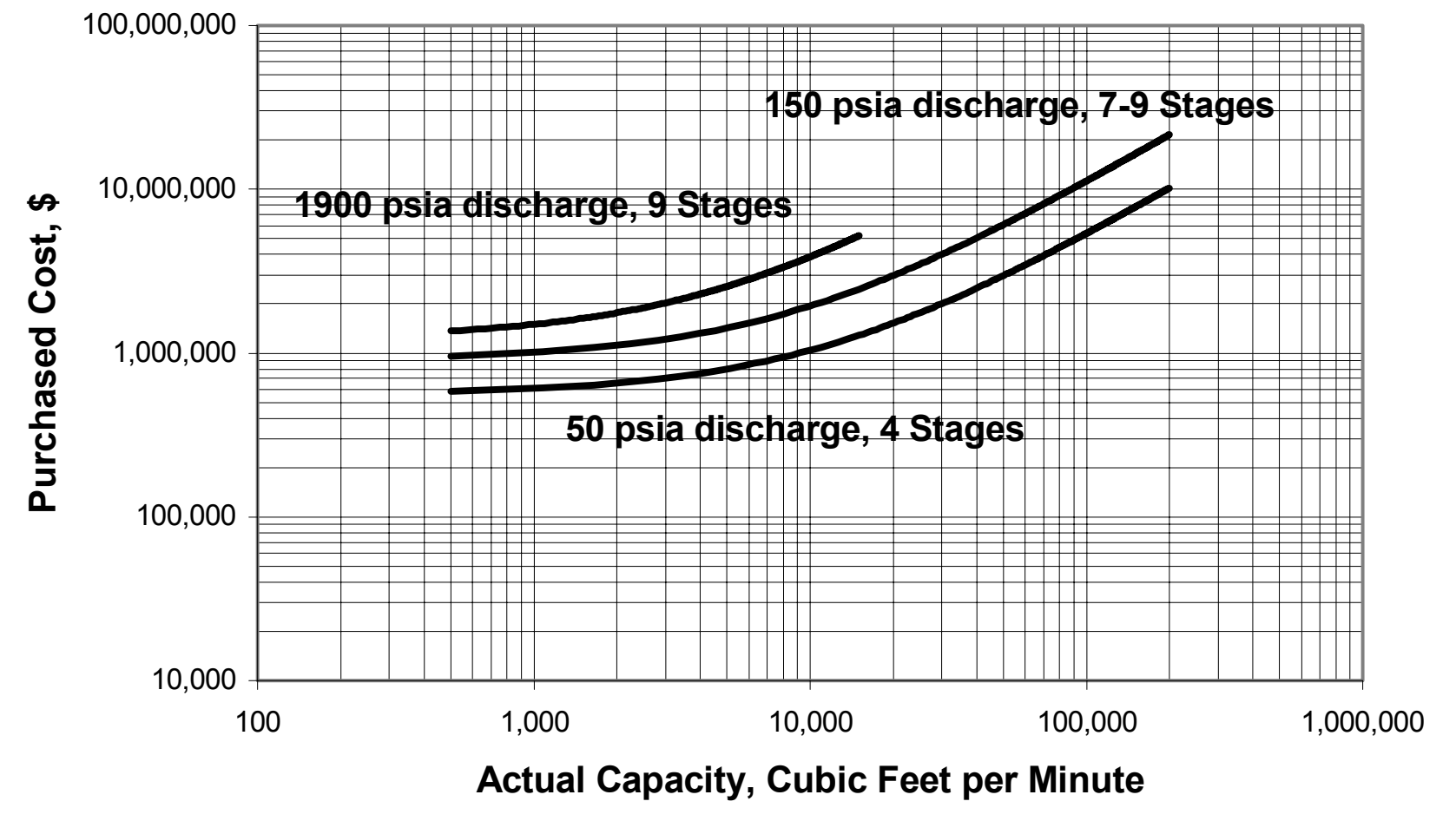




\section{Centrifugal Fan}

Description: Centrifugal fans move gas through a low pressure drop system. Maximum pressure rise is about 2 PSI.

\section{Design Basis:}

$1^{\text {st }}$ Quarter 1998 Dollars

Material:

Carbon Steel

Power:

Speed:

1.5 - 300 Horsepower

Exit Pressure: 1800 RPM

6 In $\mathrm{H} 2 \mathrm{O}$

\section{Centrifugal Fan \\ Purchased Equipment Cost}

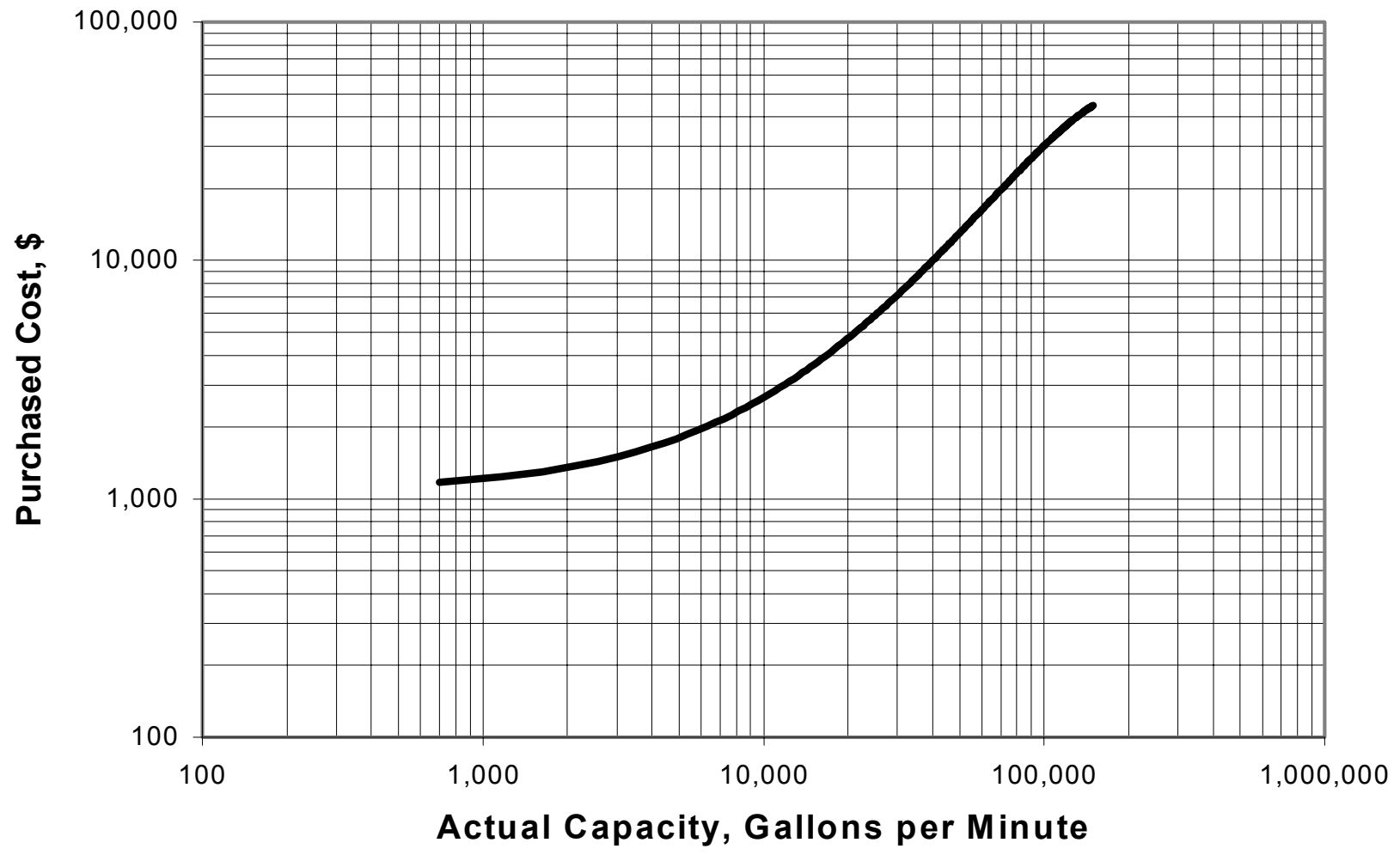




\section{Rotary Blower}

Description: This general-purpose blower includes inlet and discharge silencers. The casing of the rotary blower is cast iron and the impellers are ductile iron.

\section{Design Basis:}

$1^{\text {st }}$ Quarter 1998 Dollars

Material:

Carbon Steel

Power:

Speed:

5 - 200 Horsepower

Exit Pressure:

1800 RPM

8 psig

\section{Rotary Blower}

Purchased Equipment Cost

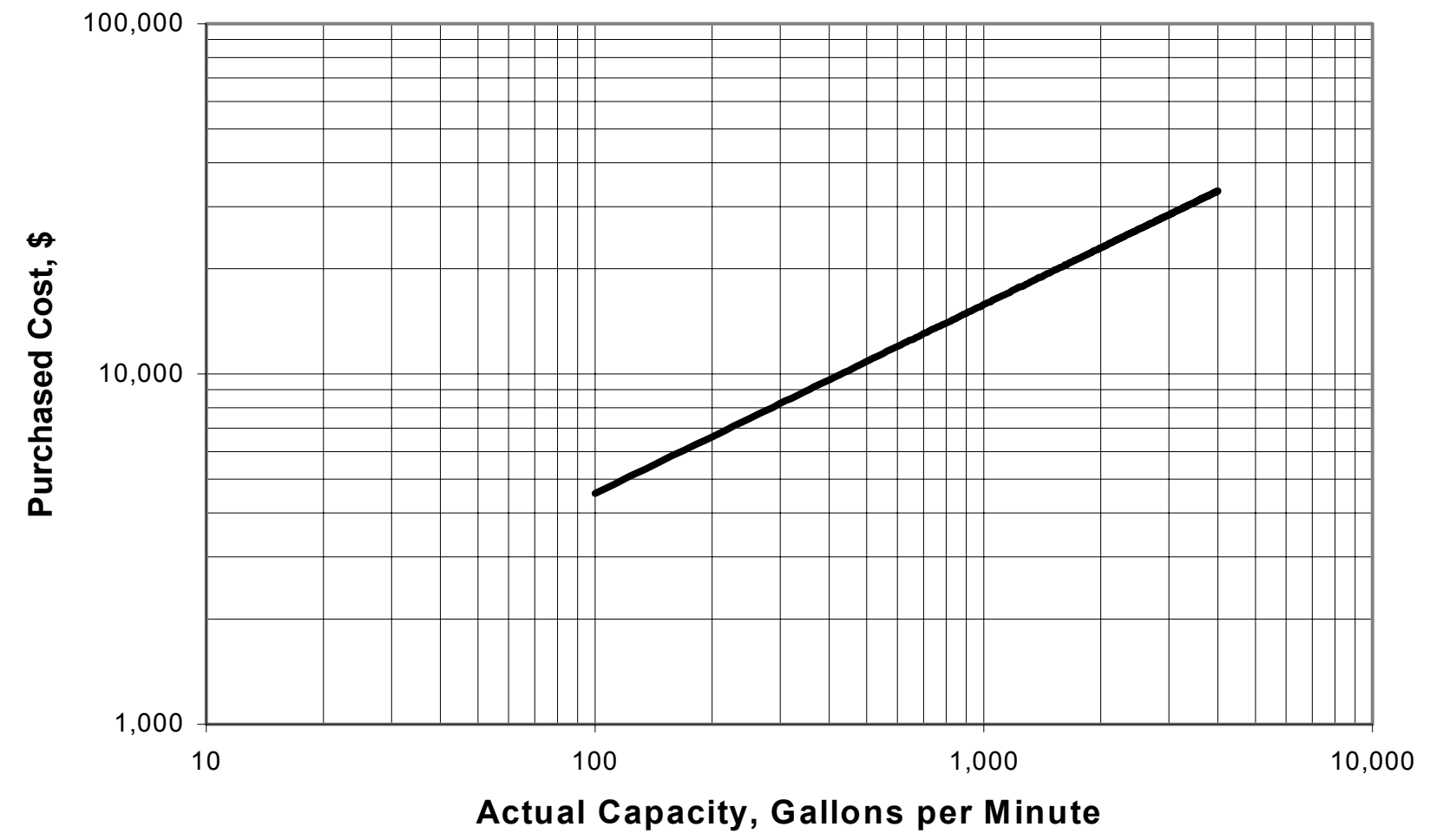




\section{Gas Turbine}

Description: Gas turbine includes fuel gas combustion chamber and multi-stage turbine expander.

\section{Design Basis:}

$1^{\text {st }}$ Quarter 1998 Dollars

Material:

Carbon Steel

\section{Gas Turbine \\ Purchased Equipment Cost}

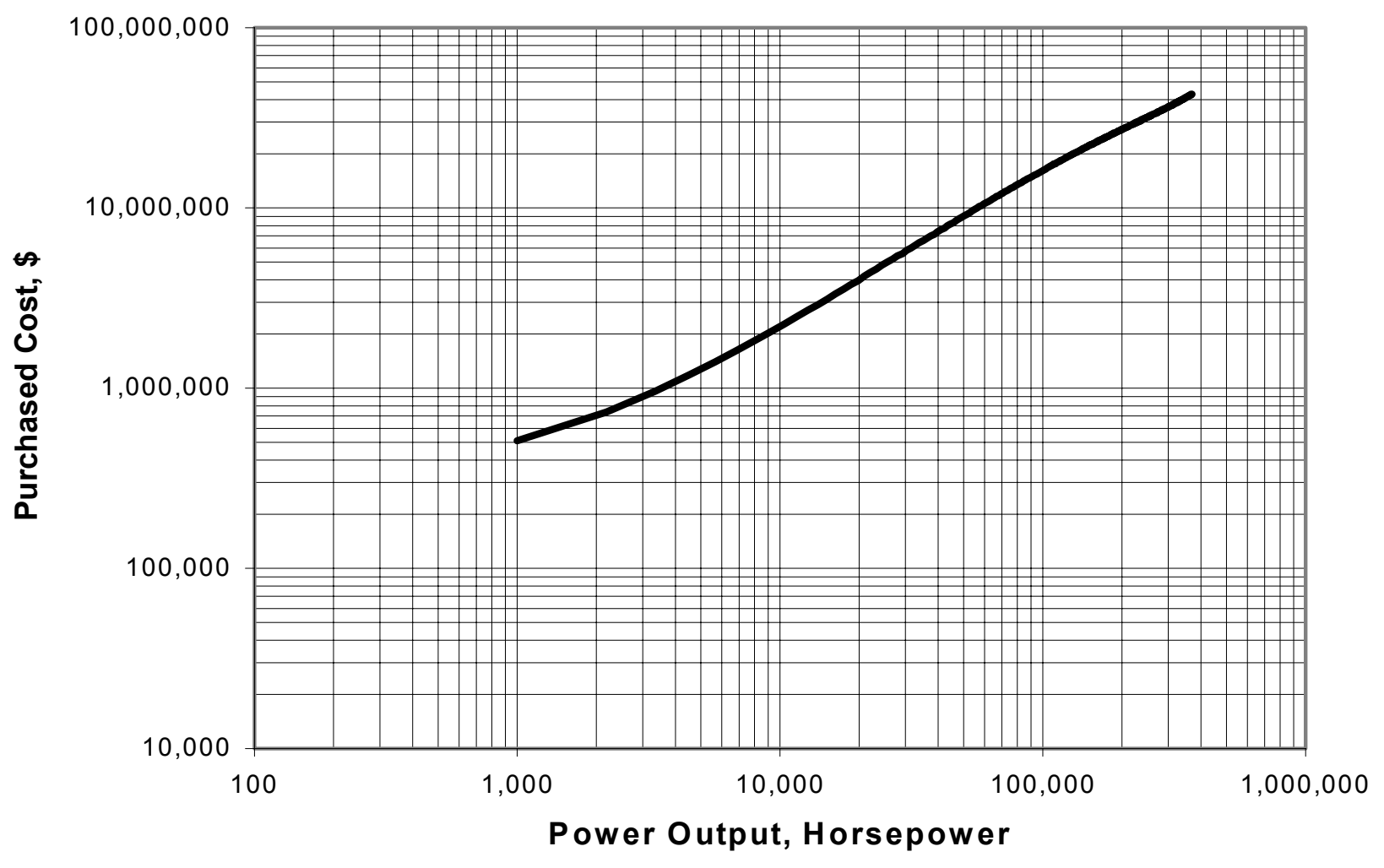


Steam Turbine - under 1000 Horsepower

Description: Steam turbine driver includes condenser and accessories.

Design Basis:

$1^{\text {st }}$ Quarter 1998 Dollars

Material:

Carbon Steel

Steam Pressure: $\quad 400$ psig

Speed: 3600 RPM

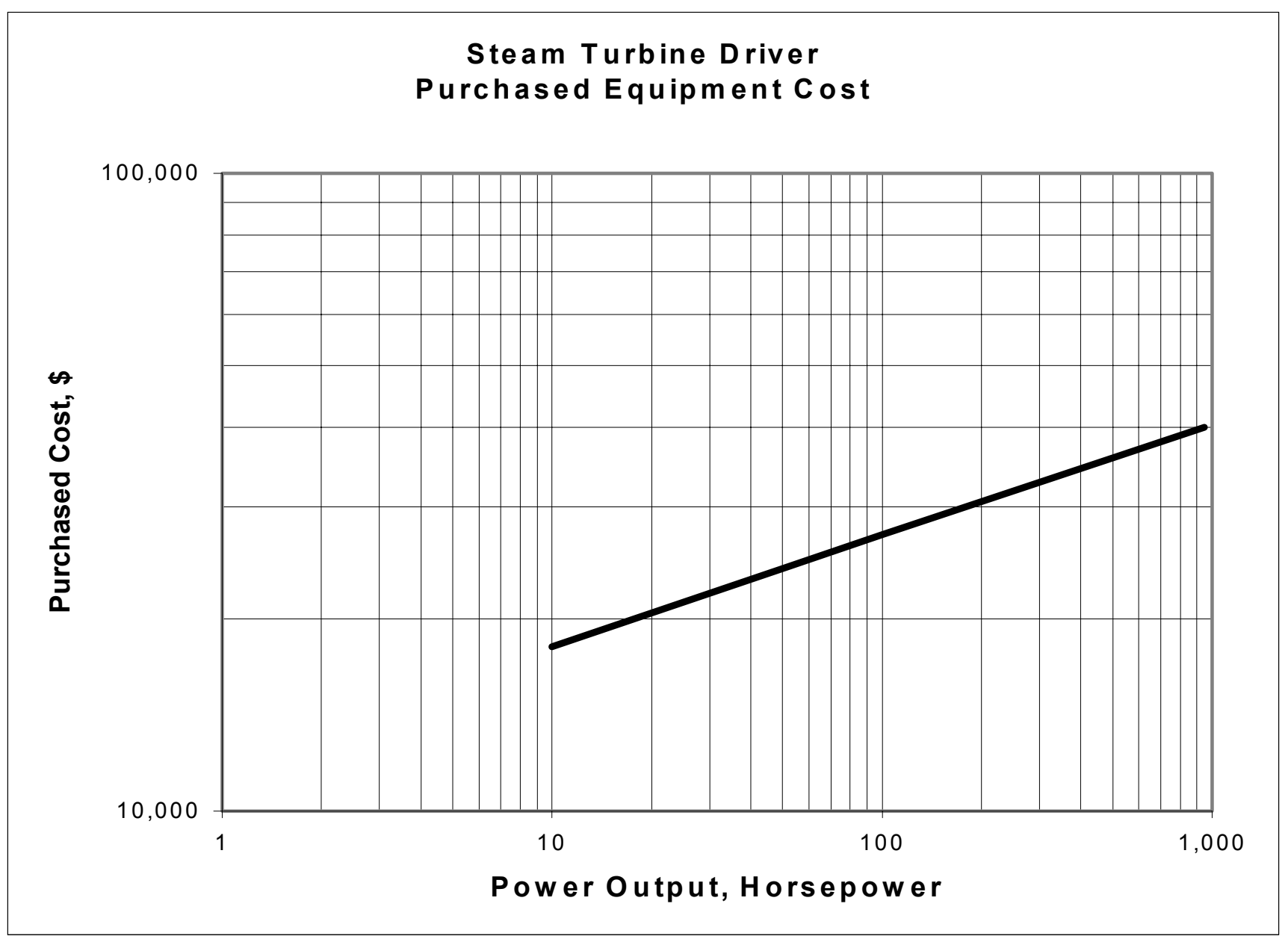


Steam Turbine - over 1000 Horsepower

Description: Steam turbine driver includes condenser and accessories.

\section{Design Basis:}

$1^{\text {st }}$ Quarter 1998 Dollars

Material:

Carbon Steel

Steam Pressure: $\quad 400$ psig

Speed: 3600 RPM

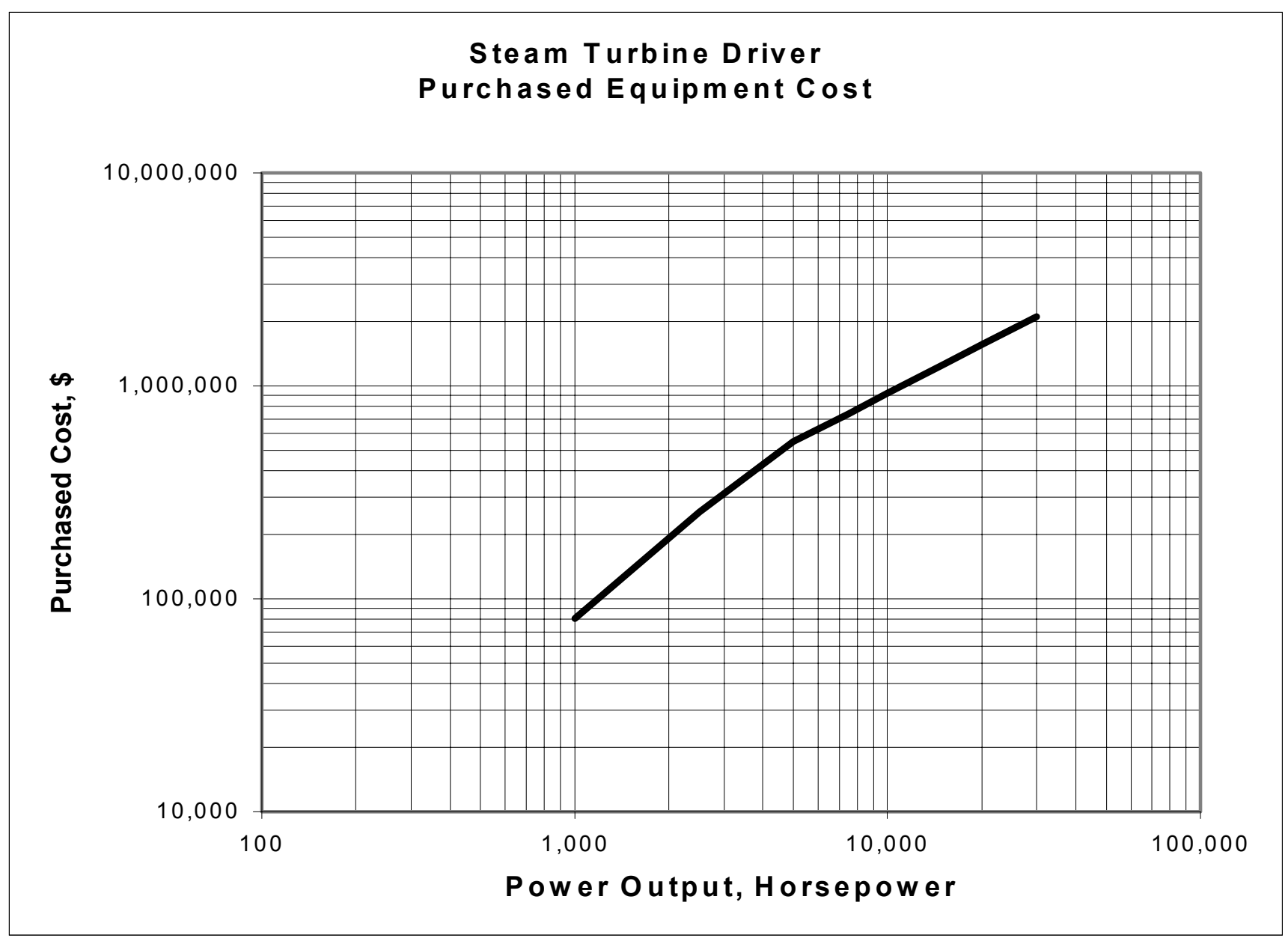


Table 2

Distributive Factors for Bulk Materials - Solids Handling Processes

\begin{tabular}{|c|c|c|c|}
\hline Temperature & & $\begin{array}{c}\leq 400{ }^{\circ} \mathrm{F} \\
(\%)\end{array}$ & $\begin{array}{c}>400{ }^{\circ} \mathrm{F} \\
(\%)\end{array}$ \\
\hline Foundations & $\begin{array}{l}\text { Material } \\
\text { Labor }\end{array}$ & $\begin{array}{r}4 \\
133\end{array}$ & $\begin{array}{r}5 \\
133\end{array}$ \\
\hline Structural Steel & $\begin{array}{l}\text { Material } \\
\text { Labor }\end{array}$ & $\begin{array}{r}4 \\
50\end{array}$ & $\begin{array}{r}2 \\
100\end{array}$ \\
\hline Buildings & $\begin{array}{l}\text { Material } \\
\text { Labor }\end{array}$ & $\begin{array}{r}2 \\
100\end{array}$ & $\begin{array}{r}2 \\
100\end{array}$ \\
\hline Insulation & $\begin{array}{l}\text { Material } \\
\text { Labor }\end{array}$ & --- & $\begin{array}{l}1.5 \\
150\end{array}$ \\
\hline Instruments & $\begin{array}{l}\text { Material } \\
\text { Labor }\end{array}$ & $\begin{array}{r}6 \\
10\end{array}$ & $\begin{array}{r}6 \\
40\end{array}$ \\
\hline Electrical & $\begin{array}{l}\text { Material } \\
\text { Labor }\end{array}$ & $\begin{array}{r}9 \\
75\end{array}$ & $\begin{array}{r}9 \\
75\end{array}$ \\
\hline Piping & $\begin{array}{l}\text { Material } \\
\text { Labor }\end{array}$ & $\begin{array}{r}5 \\
50\end{array}$ & $\begin{array}{r}5 \\
50\end{array}$ \\
\hline Painting & $\begin{array}{l}\text { Material } \\
\text { Labor }\end{array}$ & $\begin{array}{l}0.5 \\
300\end{array}$ & $\begin{array}{l}0.5 \\
300\end{array}$ \\
\hline Miscellaneous & $\begin{array}{l}\text { Material } \\
\text { Labor }\end{array}$ & $\begin{array}{r}3 \\
80\end{array}$ & $\begin{array}{r}4 \\
80\end{array}$ \\
\hline
\end{tabular}


Table 3

Distributive Factors for Bulk Materials - Solids - Gas Processes

\begin{tabular}{|c|c|c|c|c|c|}
\hline \multirow{2}{*}{$\begin{array}{l}\text { Temperature } \\
\text { Pressure }\end{array}$} & \multicolumn{3}{|c|}{$\leq 400^{\circ} \mathrm{F}$} & \multicolumn{2}{|c|}{$>400^{\circ} \mathrm{F}$} \\
\hline & & $\begin{array}{c}\leq 150 \text { psig } \\
(\%)\end{array}$ & $\begin{array}{c}>150 \text { psig } \\
(\%)\end{array}$ & $\begin{array}{c}\leq 150 \text { psig } \\
(\%)\end{array}$ & $\begin{array}{l}>150 \text { psig } \\
(\%)\end{array}$ \\
\hline Foundations & $\begin{array}{l}\text { Material } \\
\text { Labor }\end{array}$ & $\begin{array}{r}5 \\
133\end{array}$ & $\begin{array}{r}6 \\
133\end{array}$ & $\begin{array}{r}6 \\
133\end{array}$ & $\begin{array}{r}6 \\
133\end{array}$ \\
\hline Structural Steel & $\begin{array}{l}\text { Material } \\
\text { Labor }\end{array}$ & $\begin{array}{r}4 \\
100\end{array}$ & $\begin{array}{r}4 \\
100\end{array}$ & $\begin{array}{r}5 \\
50\end{array}$ & $\begin{array}{r}6 \\
50\end{array}$ \\
\hline Buildings & $\begin{array}{l}\text { Material } \\
\text { Labor }\end{array}$ & $\begin{array}{r}2 \\
100\end{array}$ & $\begin{array}{r}2 \\
50\end{array}$ & $\begin{array}{r}5 \\
50\end{array}$ & $\begin{array}{c}4 \\
100\end{array}$ \\
\hline Insulation & $\begin{array}{l}\text { Material } \\
\text { Labor }\end{array}$ & $\begin{array}{r}1 \\
150\end{array}$ & $\begin{array}{r}1 \\
150\end{array}$ & $\begin{array}{r}2 \\
150\end{array}$ & $\begin{array}{r}2 \\
150\end{array}$ \\
\hline Instruments & $\begin{array}{l}\text { Material } \\
\text { Labor }\end{array}$ & $\begin{array}{r}2 \\
40\end{array}$ & $\begin{array}{r}7 \\
40\end{array}$ & $\begin{array}{r}7 \\
40\end{array}$ & $\begin{array}{r}8 \\
75\end{array}$ \\
\hline Electrical & $\begin{array}{l}\text { Material } \\
\text { Labor }\end{array}$ & $\begin{array}{r}6 \\
75\end{array}$ & $\begin{array}{r}8 \\
75\end{array}$ & $\begin{array}{r}7 \\
75\end{array}$ & $\begin{array}{r}8 \\
75\end{array}$ \\
\hline Piping & $\begin{array}{l}\text { Material } \\
\text { Labor }\end{array}$ & $\begin{array}{l}35 \\
50\end{array}$ & $\begin{array}{l}40 \\
50\end{array}$ & $\begin{array}{l}40 \\
50\end{array}$ & $\begin{array}{l}40 \\
50\end{array}$ \\
\hline Painting & $\begin{array}{l}\text { Material } \\
\text { Labor }\end{array}$ & $\begin{array}{l}0.5 \\
300\end{array}$ & $\begin{array}{l}0.5 \\
300\end{array}$ & $\begin{array}{l}0.5 \\
300\end{array}$ & $\begin{array}{l}0.5 \\
300\end{array}$ \\
\hline Miscellaneous & $\begin{array}{l}\text { Material } \\
\text { Labor }\end{array}$ & $\begin{array}{l}3.5 \\
80\end{array}$ & $\begin{array}{r}4 \\
80\end{array}$ & $\begin{array}{r}4 \\
80\end{array}$ & $\begin{array}{l}4.5 \\
80\end{array}$ \\
\hline
\end{tabular}




\section{Table 4}

Distributive Factors for Bulk Materials - Liquid and Slurry Systems

\begin{tabular}{|c|c|c|c|}
\hline Pressure & & $\begin{array}{c}\leq 150 \text { psig } \\
(\%)\end{array}$ & $\begin{array}{c}>150 \text { psig } \\
(\%)\end{array}$ \\
\hline Foundations & $\begin{array}{l}\text { Material } \\
\text { Labor }\end{array}$ & $\begin{array}{r}5 \\
133\end{array}$ & $\begin{array}{r}6 \\
133\end{array}$ \\
\hline Structural Steel & $\begin{array}{l}\text { Material } \\
\text { Labor }\end{array}$ & $\begin{array}{r}4 \\
50\end{array}$ & $\begin{array}{r}5 \\
50\end{array}$ \\
\hline Buildings & $\begin{array}{l}\text { Material } \\
\text { Labor }\end{array}$ & $\begin{array}{r}3 \\
100\end{array}$ & $\begin{array}{r}3 \\
100\end{array}$ \\
\hline Insulation & $\begin{array}{l}\text { Material } \\
\text { Labor }\end{array}$ & $\begin{array}{r}1 \\
150\end{array}$ & $\begin{array}{r}3 \\
150\end{array}$ \\
\hline Instruments & $\begin{array}{l}\text { Material } \\
\text { Labor }\end{array}$ & $\begin{array}{r}6 \\
40\end{array}$ & $\begin{array}{r}7 \\
40\end{array}$ \\
\hline Electrical & $\begin{array}{l}\text { Material } \\
\text { Labor }\end{array}$ & $\begin{array}{r}8 \\
75\end{array}$ & $\begin{array}{r}9 \\
75\end{array}$ \\
\hline Piping & $\begin{array}{l}\text { Material } \\
\text { Labor }\end{array}$ & $\begin{array}{l}30 \\
50\end{array}$ & $\begin{array}{l}35 \\
50\end{array}$ \\
\hline Painting & $\begin{array}{l}\text { Material } \\
\text { Labor }\end{array}$ & $\begin{array}{l}0.5 \\
300\end{array}$ & $\begin{array}{l}0.5 \\
300\end{array}$ \\
\hline Miscellaneous & $\begin{array}{l}\text { Material } \\
\text { Labor }\end{array}$ & $\begin{array}{r}4 \\
80\end{array}$ & $\begin{array}{r}5 \\
80\end{array}$ \\
\hline
\end{tabular}


Table 5

Distributive Factors for Bulk Materials - Gas Processes

\begin{tabular}{|c|c|c|c|c|c|}
\hline \multirow{2}{*}{$\begin{array}{l}\text { Temperature } \\
\text { Pressure }\end{array}$} & \multicolumn{3}{|c|}{$\leq 400^{\circ} \mathrm{F}$} & \multicolumn{2}{|c|}{$>400^{\circ} \mathrm{F}$} \\
\hline & & $\begin{array}{c}\leq 150 \mathrm{psig} \\
(\%)\end{array}$ & $\begin{array}{c}>150 \text { psig } \\
(\%)\end{array}$ & $\begin{array}{c}\leq 150 \mathrm{psig} \\
(\%)\end{array}$ & $\begin{array}{c}>150 \mathrm{psig} \\
(\%)\end{array}$ \\
\hline Foundations & $\begin{array}{l}\text { Material } \\
\text { Labor }\end{array}$ & $\begin{array}{r}5 \\
133\end{array}$ & $\begin{array}{r}6 \\
133\end{array}$ & $\begin{array}{r}6 \\
133\end{array}$ & $\begin{array}{r}5 \\
133\end{array}$ \\
\hline Structural Steel & $\begin{array}{l}\text { Material } \\
\text { Labor }\end{array}$ & $\begin{array}{r}5 \\
50\end{array}$ & $\begin{array}{r}5 \\
50\end{array}$ & $\begin{array}{r}5 \\
50\end{array}$ & $\begin{array}{r}6 \\
50\end{array}$ \\
\hline Buildings & $\begin{array}{l}\text { Material } \\
\text { Labor }\end{array}$ & $\begin{array}{r}3 \\
100\end{array}$ & $\begin{array}{r}3 \\
100\end{array}$ & $\begin{array}{r}3 \\
100\end{array}$ & $\begin{array}{r}4 \\
100\end{array}$ \\
\hline Insulation & $\begin{array}{l}\text { Material } \\
\text { Labor }\end{array}$ & $\begin{array}{r}1 \\
150\end{array}$ & $\begin{array}{r}1 \\
150\end{array}$ & $\begin{array}{r}2 \\
150\end{array}$ & $\begin{array}{r}3 \\
150\end{array}$ \\
\hline Instruments & $\begin{array}{l}\text { Material } \\
\text { Labor }\end{array}$ & $\begin{array}{r}6 \\
40\end{array}$ & $\begin{array}{r}7 \\
40\end{array}$ & $\begin{array}{r}7 \\
75\end{array}$ & $\begin{array}{r}7 \\
40\end{array}$ \\
\hline Electrical & $\begin{array}{l}\text { Material } \\
\text { Labor }\end{array}$ & $\begin{array}{r}8 \\
75\end{array}$ & $\begin{array}{r}9 \\
75\end{array}$ & $\begin{array}{r}6 \\
40\end{array}$ & $\begin{array}{r}9 \\
75\end{array}$ \\
\hline Piping & $\begin{array}{l}\text { Material } \\
\text { Labor }\end{array}$ & $\begin{array}{l}45 \\
50\end{array}$ & $\begin{array}{l}40 \\
50\end{array}$ & $\begin{array}{l}40 \\
50\end{array}$ & $\begin{array}{l}40 \\
50\end{array}$ \\
\hline Painting & $\begin{array}{l}\text { Material } \\
\text { Labor }\end{array}$ & $\begin{array}{l}0.5 \\
300\end{array}$ & $\begin{array}{l}0.5 \\
300\end{array}$ & $\begin{array}{l}0.5 \\
300\end{array}$ & $\begin{array}{l}0.5 \\
300\end{array}$ \\
\hline Miscellaneous & $\begin{array}{l}\text { Material } \\
\text { Labor }\end{array}$ & $\begin{array}{r}3 \\
80\end{array}$ & $\begin{array}{r}4 \\
80\end{array}$ & $\begin{array}{r}4 \\
80\end{array}$ & $\begin{array}{r}5 \\
80\end{array}$ \\
\hline
\end{tabular}




\section{Table 6}

Distributive Labor Factors for Setting Equipment

\begin{tabular}{llll}
\hline \hline Equipment Type & $\begin{array}{l}\text { Factor } \\
(\%)\end{array}$ & Equipment Type & $\begin{array}{l}\text { Factor } \\
(\%)\end{array}$ \\
\hline Absorber & 20 & Hammermill & 25 \\
Ammonia Still & 20 & Heater & 20 \\
Ball Mill & 30 & Heat Exchanger & 20 \\
Briquetting machine & 25 & Lime Leg & 15 \\
Centrifuge & 20 & Methanator (catalytic) & 30 \\
Clarifier & 15 & Mixer & 20 \\
Coke Cutter & 15 & Precipitator & 25 \\
Coke Drum & 15 & Regenerator (packed) & 20 \\
Condenser & 20 & Retort & 30 \\
Conditioner & 20 & Rotoclone & 25 \\
Cooler & 20 & Screen & 20 \\
Crusher & 30 & Scrubber (water) & 15 \\
Cyclone & 20 & Settler & 15 \\
Decanter & 15 & Shift converter & 25 \\
Distillation column & 30 & Splitter & 15 \\
Evaporator & 20 & Storage Tank & 20 \\
Filter & 15 & Stripper & 20 \\
Fractionator & 25 & Tank & 20 \\
Furnace & 30 & Vaporizer & 20 \\
Gasifier & 30 & & \\
\hline \hline
\end{tabular}




\section{Table 7}

Factors for Converting Carbon Steel to Equivalent Alloy Costs

\begin{tabular}{lcc}
\hline \multicolumn{1}{c}{ Material } & Pumps, etc. & Other Equipment \\
\hline All Carbon Steel & 1.00 & 1.00 \\
Stainless Steel, Type 410 & 1.43 & 2.00 \\
Stainless Steel, Type 304 & 1.70 & 2.80 \\
Stainless Steel, Type 316 & 1.80 & 2.90 \\
Stainless Steel, Type 310 & 2.00 & 3.33 \\
Rubber-lined Steel & 1.43 & 1.25 \\
Bronze & 1.54 & \\
Monel & 3.33 & \\
\hline \hline
\end{tabular}

\begin{tabular}{lc}
\hline \multicolumn{1}{c}{ Material } & Heat Exchangers \\
\hline Carbon Steel Shell and Tubes & 1.00 \\
Carbon Steel Shell, Aluminum Tubes & 1.25 \\
Carbon Steel Shell, Monel Tubes & 2.08 \\
Carbon Steel Shell, 304 Stainless Steel Tubes & 1.67 \\
304 Stainless Steel Shell and Tubes & 2.86 \\
\hline \hline
\end{tabular}




\section{Cost Indexes}

Cost indexes are used to update costs from the base time, in this case First Quarter 1998 dollars, to the present time of the estimate. Cost indexes are used to give a general estimate, but can not take into account all factors. Some limitations of cost indexes include: ${ }^{3}$

1. Accuracy is very limited. Two Indexes may yield much different answers.

2. Cost indexes are based on averages. Specific cases may be much different from the average.

3. At best, $10 \%$ accuracy can be expected for periods up to 5 years.

4. For periods over 10 years, indexes are suitable only for order of magnitude estimates.

The most common indexes are Engineering News-Record Construction Cost Index, Table 8, (published in the Engineering News-Record), Marshall and Swift Equipment Cost Indexes, Table 9, (published in Chemical Engineering), Nelson-Farrar Refinery Construction Cost Index, Table 10, (published in the Oil and Gas Journal) and the Chemical Engineering Plant Cost Index, Table 11, (published in Chemical Engineering). Annual averages for each of these indexes are included in this report.

The Marshall and Swift Equipment Cost Indexes are divided into two categories, the allindustry equipment index and the process-industry equipment index. The indexes take into consideration the cost of machinery and major equipment plus costs for installation, fixtures, tools, office furniture, and other minor equipment. The Engineering NewsRecord Construction Cost Index shows the variation in the labor rates and materials costs for industrial construction. The Nelson-Farrar Refinery Construction Cost Index uses construction costs in the petroleum industry as the basis. The Chemical Engineering Plant Cost Index uses construction costs for chemical plants as the basis.

Two cost indexes, the Marshall and Swift equipment cost indexes and the Chemical Engineering plant cost indexes, give very similar results and are recommended for use with process-equipment estimates and chemical-plant investment estimates. The Engineering News-Record construction cost index, relative with time, has increased much more rapidly than the other two because it does not include a productivity improvement factor. Similarly, the Nelson-Farrar refinery construction index has shown a very large increase with time and should be used with caution and only for refinery construction. ${ }^{4}$

\footnotetext{
${ }^{3}$ Humphreys, Dr. Kenneth K. PE CCE, "Preliminary Capital and Operating Cost Estimating (for the Process and Utility Industries)," course notes.

${ }^{4}$ Peters, Max S. and Klaus D. Timmerhaus, "Plant Design and Economics for Chemical Engineers" McGraw-Hill, Inc. 1991.
} 


\section{Table 8}

Engineering News Record Construction Cost Index

Published in the Engineering News-Record

\begin{tabular}{|c|c|}
\hline Year & Annual Average \\
\hline 1913 & 100 \\
\hline 1960 & 824 \\
\hline 1965 & 971 \\
\hline 1970 & 1381 \\
\hline 1975 & 2212 \\
\hline 1980 & 3237 \\
\hline 1985 & 4195 \\
\hline 1990 & 4732 \\
\hline 1995 & 5471 \\
\hline 1996 & 5620 \\
\hline 1997 & 5825 \\
\hline 1998 & 5920 \\
\hline 1999 & 6060 \\
\hline 2000 & 6222 \\
\hline \multicolumn{2}{|l|}{2001} \\
\hline January & 6281 \\
\hline February & 6273 \\
\hline March & 6280 \\
\hline April & 6286 \\
\hline May & 6288 \\
\hline
\end{tabular}




\section{Table 9}

Marshall and Swift Installed-Equipment Index

Published in Chemical Engineering

\begin{tabular}{lcc}
\hline \hline & \multicolumn{2}{c}{ Annual Average } \\
Year & All Industry & Process Industry \\
\hline $\mathbf{1 9 2 6}$ & $\mathbf{1 0 0}$ & $\mathbf{1 0 0}$ \\
1964 & 242 & 241 \\
1965 & 245 & 244 \\
1970 & 303 & 301 \\
1975 & 444 & 452 \\
1980 & 560 & 675 \\
1985 & 790 & 813 \\
1990 & 915 & 935 \\
1995 & 1027.5 & 1037.4 \\
1996 & 1039.2 & 1051.3 \\
1997 & 1056.8 & 1068.3 \\
1998 & 1061.9 & 1075.9 \\
1st Quarter & 1061.2 & 1074.6 \\
2nd Quarter & 1061.8 & 1075.2 \\
3rd Quarter & 1062.4 & 1077.2 \\
4th Quarter & 1062.3 & 1076.6 \\
1999 & 1068.3 & 1083.1 \\
1st Quarter & 1062.7 & 1078.8 \\
2nd Quarter & 1065.0 & 1080.7 \\
3rd Quarter & 1069.9 & 1084.0 \\
4th Quarter & 1075.6 & 1088.7 \\
2000 & 1089.0 & 1102.7 \\
1st Quarter & 1080.6 & 1093.5 \\
2nd Quarter & 1089.0 & 1102.2 \\
3rd Quarter & 1092.0 & 1106.3 \\
4th Quarter & 1094.5 & 1108.7 \\
2001 & & \\
1st Quarter & 1092.8 & \\
\hline \hline
\end{tabular}


Table 10

Nelson-Farrar Refinery Construction Index

Published in the Oil and Gas Journal

\begin{tabular}{ccccc}
\hline \hline Year & $\begin{array}{c}\text { Annual } \\
\text { Average }\end{array}$ & $\begin{array}{c}\text { Pumps, } \\
\text { Compressors, etc }\end{array}$ & $\begin{array}{c}\text { Heat } \\
\text { Exchangers }\end{array}$ & $\begin{array}{c}\text { Misc. } \\
\text { Equipment } \\
\text { Average }\end{array}$ \\
\hline $\mathbf{1 9 4 6}$ & $\mathbf{1 0 0}$ & & & \\
1964 & 252 & & & \\
1965 & 261 & & & \\
1970 & 365 & & & \\
1975 & 576 & & 618.7 & 578.1 \\
1980 & 823 & 777.3 & 520 & 673.4 \\
1985 & 1074 & 969.9 & 755.7 & 797.5 \\
1990 & 1225.7 & 1125.6 & 758.6 & 879.5 \\
1995 & 1392.1 & 1316.7 & 793.3 & 903.5 \\
1996 & 1418.9 & 1354.5 & 773.6 & 910.5 \\
1997 & 1449.2 & 1383.9 & 841.1 & 933.2 \\
1998 & 1477.6 & 1406.7 & 715.8 & 920.3 \\
1999 & 1497.2 & 1433.5 & 662.2 & 917.8 \\
2000 & 1542.7 & 1456.4 & & \\
2001 & & & 722.7 & 936.2 \\
January & 1565.9 & 1473.2 & 722.7 & 937.1 \\
February & 1563.6 & 1478.9 & & \\
\hline \hline
\end{tabular}




\section{Table 11}

Chemical Engineering Plant Cost Index

Published in Chemical Engineering

\begin{tabular}{cc}
\hline \hline Year & Annual Average \\
\hline $\mathbf{1 9 5 7 - 5 9}$ & $\mathbf{1 0 0}$ \\
1964 & 103 \\
1965 & 104 \\
1970 & 126 \\
1975 & 182 \\
1980 & 261 \\
1985 & 325 \\
1990 & 357.6 \\
1995 & 381.1 \\
1996 & 381.8 \\
1997 & 386.5 \\
1998 & 389.5 \\
1999 & 390.6 \\
2000 & 394.1 \\
2001 & \\
January & 395.4 \\
\hline \hline
\end{tabular}




\section{Appendix A}

The following is an example of the usage of the cost curves and tables to estimate the installed cost of a 5,000 square foot gas-gas shell and tube heat exchanger with a design temperature of $650^{\circ} \mathrm{F}$ and a design pressure of $150 \mathrm{psig}$.

From the chart on page 16, the estimated purchased equipment cost is $\$ 62,000$. From Table 6 , the factor for setting a heat exchanger is $20 \%$. Column 3 of Table 5 is used to estimate the bulk material and labor costs.

Bare cost:

$\$ 62,000$

Setting Cost: $\quad \$ 62,000 * 0.2 \quad \$ 12,400$

Bulk Installations:

Foundations

$\begin{array}{lll}\text { Material } & \$ 62,000 * 0.06 & \$ 3,720 \\ \text { Labor } & \$ 3,720 * 1.33 & \$ 4,948\end{array}$

Structural Steel

Material

$\$ 62,000 * 0.05$

$\$ 3,100$

Labor

$\$ 3,100 * 0.5$

$\$ 1,550$

Buildings

Material $\$ 62,000 * 0.03 \quad \$ 1,860$

Labor $\quad \$ 1,860 * 1.0 \quad \$ 1,860$

Insulation

Material

$\$ 62,000 * 0.02$

$\$ 1,240$

Labor

$\$ 1,240 * 1.5$

$\$ 1,860$

Instruments

Material $\$ 62,000 * 0.07 \quad \$ 4,340$

Labor $\$ 4,340 * 0.75 \quad \$ 3,255$

Electrical

Material \$62,000*0.06 \$3,720

Labor $\$ 3,720 * 0.4 \quad \$ 1,488$

Piping

Material $\$ 62,000 * 0.4 \quad \$ 24,800$

Labor $\quad \$ 24,800 * 0.5 \quad \$ 12,400$

Painting

Material $\$ 62,000 * 0.005 \quad \$ 310$

Labor $\$ 310 * 3.0 \quad \$ 930$

Miscellaneous

Material $\$ 62,000 * 0.04 \quad \$ 2,480$

Labor $\$ 2,480 * 0.8 \quad \$ 1,984$

Total Installed Cost: $\$ 150,245$

From ICARUS-generated results (page 59):

Purchased Equipment Cost

$\$ 62,100$

Total Installed Cost

$\$ 141,800$ 


\section{Appendix B}

Vertical Vessels

$1^{\text {st }}$ Quarter 1998 dollars

\begin{tabular}{|r|r|r|r|r|r|}
\hline \multicolumn{7}{|c|}{15 psig } \\
\hline $\begin{array}{c}\text { Diameter } \\
\text { (Feet) }\end{array}$ & $\begin{array}{c}\text { Height } \\
\text { (Feet) }\end{array}$ & $\begin{array}{c}\text { Capacity } \\
\text { (Gallons) }\end{array}$ & $\begin{array}{c}\text { Total } \\
\text { Weight } \\
\text { (Pounds) }\end{array}$ & $\begin{array}{c}\text { Purchased } \\
\text { Equipment } \\
\text { Cost (\$) }\end{array}$ & $\begin{array}{r}\text { Installed } \\
\text { Cost (\$) }\end{array}$ \\
\hline 2.5 & 2.7 & 100 & 1,000 & $\$ 6,400$ & $\$ 51,800$ \\
\hline 3.0 & 4.7 & 250 & 1,400 & $\$ 7,400$ & $\$ 61,000$ \\
\hline 4.0 & 5.3 & 500 & 2,000 & $\$ 9,800$ & $\$ 68,400$ \\
\hline 4.0 & 8.0 & 750 & 2,700 & $\$ 12,200$ & $\$ 89,700$ \\
\hline 5.0 & 6.8 & 1,000 & 3,000 & $\$ 13,000$ & $\$ 96,000$ \\
\hline 6.0 & 9.5 & 2,000 & 4,200 & $\$ 16,500$ & $\$ 122,300$ \\
\hline 7.0 & 10.4 & 3,000 & 5,200 & $\$ 18,000$ & $\$ 132,300$ \\
\hline 7.0 & 13.9 & 4,000 & 6,300 & $\$ 18,600$ & $\$ 135,100$ \\
\hline 8.0 & 13.3 & 5,000 & 7,100 & $\$ 21,000$ & $\$ 139,700$ \\
\hline
\end{tabular}

\begin{tabular}{|r|r|r|r|r|r|}
\hline \multicolumn{7}{|c|}{150 psig } \\
\hline $\begin{array}{c}\text { Diameter } \\
\text { (Feet) }\end{array}$ & $\begin{array}{c}\text { Height } \\
\text { (Feet) }\end{array}$ & $\begin{array}{c}\text { Capacity } \\
\text { (Gallons) }\end{array}$ & $\begin{array}{c}\text { Total } \\
\text { Weight } \\
\text { (Pounds) }\end{array}$ & $\begin{array}{c}\text { Purchased } \\
\text { Equipment } \\
\text { Cost (\$) }\end{array}$ & $\begin{array}{r}\text { Installed } \\
\text { Cost (\$) }\end{array}$ \\
\hline 2.5 & 2.7 & 100 & 1,300 & $\$ 7,000$ & $\$ 48,800$ \\
\hline 3.0 & 4.7 & 250 & 1,800 & $\$ 8,300$ & $\$ 52,500$ \\
\hline 4.0 & 5.3 & 500 & 2,800 & $\$ 11,300$ & $\$ 60,900$ \\
\hline 4.0 & 8.0 & 750 & 3,600 & $\$ 13,700$ & $\$ 76,900$ \\
\hline 5.0 & 6.8 & 1,000 & 4,500 & $\$ 15,600$ & $\$ 84,800$ \\
\hline 6.0 & 9.5 & 2,000 & 7,000 & $\$ 20,900$ & $\$ 100,700$ \\
\hline 7.0 & 10.4 & 3,000 & 9,600 & $\$ 24,200$ & $\$ 112,800$ \\
\hline 7.0 & 13.9 & 4,000 & 11,400 & $\$ 24,900$ & $\$ 115,800$ \\
\hline 8.0 & 13.3 & 5,000 & 14,200 & $\$ 30,500$ & $\$ 124,000$ \\
\hline
\end{tabular}


Horizontal Vessels

$1^{\text {st }}$ Quarter 1998 dollars

\begin{tabular}{|r|r|r|r|r|r|}
\hline \multicolumn{7}{|c|}{$\begin{array}{c}\text { 15 psig } \\
\text { (Feet) }\end{array}$} & $\begin{array}{c}\text { Length } \\
\text { (Feet) }\end{array}$ & $\begin{array}{c}\text { Capacity } \\
\text { (Gallons) }\end{array}$ & $\begin{array}{c}\text { Total } \\
\text { Weight } \\
\text { (Pounds) }\end{array}$ & $\begin{array}{c}\text { Purchased } \\
\text { Equipment } \\
\text { Cost } \mathbf{( \$ )}\end{array}$ & $\begin{array}{c}\text { Installed } \\
\text { Cost (\$) }\end{array}$ \\
\hline 2.0 & 4.3 & 100 & 1,100 & $\$ 5,700$ & $\$ 51,900$ \\
\hline 2.5 & 6.8 & 250 & 1,500 & $\$ 7,400$ & $\$ 62,200$ \\
\hline 3.0 & 9.5 & 500 & 2,200 & $\$ 8,900$ & $\$ 79,600$ \\
\hline 4.0 & 8.0 & 750 & 2,600 & $\$ 10,200$ & $\$ 81,600$ \\
\hline 4.0 & 10.6 & 1,000 & 3,000 & $\$ 11,200$ & $\$ 88,500$ \\
\hline 6.0 & 14.2 & 3,000 & 5,600 & $\$ 17,500$ & $\$ 24,600$ \\
\hline 7.0 & 17.4 & 5,000 & 7,600 & $\$ 21,800$ & $\$ 32,300$ \\
\hline 8.0 & 18.6 & 7,000 & 9,400 & $\$ 24,800$ & $\$ 144,800$ \\
\hline 9.0 & 21.0 & 10,000 & 11,500 & $\$ 29,500$ & $\$ 153,100$ \\
\hline 11.0 & 35.2 & 25,000 & 21,500 & $\$ 40,100$ & $\$ 202,600$ \\
\hline 14.0 & 43.4 & 50,000 & 33,300 & $\$ 58,200$ & $\$ 251,500$ \\
\hline 14.5 & 60.7 & 75,000 & 47,000 & $\$ 76,400$ & $\$ 304,900$ \\
\hline 14.5 & 81.0 & 100,000 & 59,400 & $\$ 94,800$ & $\$ 383,500$ \\
\hline
\end{tabular}

\begin{tabular}{|r|r|r|r|r|r|}
\hline \multicolumn{7}{|c|}{150 psig } \\
\hline $\begin{array}{c}\text { Diameter } \\
\text { (Feet) }\end{array}$ & $\begin{array}{c}\text { Length } \\
\text { (Feet) }\end{array}$ & $\begin{array}{c}\text { Capacity } \\
\text { (Gallons) }\end{array}$ & $\begin{array}{c}\text { Total } \\
\text { Weight } \\
\text { (Pounds) }\end{array}$ & $\begin{array}{c}\text { Purchased } \\
\text { Equipment } \\
\text { Cost } \mathbf{( \$ )}\end{array}$ & $\begin{array}{c}\text { Installed } \\
\text { Cost } \mathbf{( \$ )}\end{array}$ \\
\hline 2.0 & 4.3 & 100 & 1,400 & $\$ 6,300$ & $\$ 48,900$ \\
\hline 2.5 & 6.8 & 250 & 1,800 & $\$ 8,000$ & $\$ 53,200$ \\
\hline 3.0 & 9.5 & 500 & 2,500 & $\$ 9,700$ & $\$ 66,000$ \\
\hline 4.0 & 8.0 & 750 & 3,500 & $\$ 12,000$ & $\$ 69,200$ \\
\hline 4.0 & 10.6 & 1,000 & 4,000 & $\$ 13,100$ & $\$ 76,400$ \\
\hline 6.0 & 14.2 & 3,000 & 8,900 & $\$ 23,500$ & $\$ 104,800$ \\
\hline 7.0 & 17.4 & 5,000 & 13,500 & $\$ 32,100$ & $\$ 117,200$ \\
\hline 8.0 & 18.6 & 7,000 & 18,300 & $\$ 39,900$ & $\$ 148,000$ \\
\hline 9.0 & 21.0 & 10,000 & 24,800 & $\$ 51,800$ & $\$ 163,800$ \\
\hline 11.0 & 35.2 & 25,000 & 54,100 & $\$ 90,300$ & $\$ 267,800$ \\
\hline 14.0 & 43.4 & 50,000 & 101,900 & $\$ 160,400$ & $\$ 373,200$ \\
\hline 14.5 & 60.7 & 75,000 & 155,000 & $\$ 230,300$ & $\$ 482,200$ \\
\hline 14.5 & 81.0 & 100,000 & 198,700 & $\$ 285,700$ & $\$ 606,700$ \\
\hline
\end{tabular}


Storage Tanks

$1^{\text {st }}$ Quarter 1998 dollars

\begin{tabular}{|c|c|c|c|r|r|}
\hline $\begin{array}{c}\text { Diameter } \\
\text { (Feet) }\end{array}$ & $\begin{array}{c}\text { Height } \\
\text { (Feet) }\end{array}$ & $\begin{array}{c}\text { Total } \\
\text { Weight } \\
\text { (Pounds) }\end{array}$ & $\begin{array}{c}\text { Capacity } \\
\text { (Gallons) }\end{array}$ & $\begin{array}{c}\text { Purchased } \\
\text { Equipment } \\
\text { Cost } \\
\mathbf{( \$ )}\end{array}$ & $\begin{array}{c}\text { Installed } \\
\text { Cost } \\
\mathbf{( \$ )}\end{array}$ \\
\hline \multicolumn{2}{|c|}{ Floating Roof } & & & & \\
\hline 17.0 & 32.0 & 41,300 & 50,000 & $\$ 118,000$ & $\$ 163,400$ \\
\hline 20.0 & 32.0 & 46,700 & 75,000 & $\$ 128,200$ & $\$ 180,700$ \\
\hline 24.0 & 32.0 & 55,000 & 100,000 & $\$ 143,200$ & $\$ 205,100$ \\
\hline 37.0 & 32.0 & 89,300 & 250,000 & $\$ 197,700$ & $\$ 250,000$ \\
\hline 47.0 & 40.0 & 142,400 & 500,000 & $\$ 267,800$ & $\$ 332,400$ \\
\hline 57.0 & 40.0 & 195,000 & 750,000 & $\$ 335,700$ & $\$ 411,700$ \\
\hline 66.0 & 40.0 & 245,700 & $1,000,000$ & $\$ 396,600$ & $\$ 480,200$ \\
\hline 134.0 & 48.0 & 858,900 & $5,000,000$ & $\$ 1,061,200$ & $\$ 1,250,900$ \\
\hline 175.0 & 56.0 & $2,219,100$ & $10,000,000$ & $\$ 2,273,000$ & $\$ 2,564,300$ \\
\hline Cone Roof & & & & \\
\hline 17.0 & 32.0 & 21,000 & 50,000 & $\$ 42,400$ & $\$ 87,800$ \\
\hline 20.0 & 32.0 & 26,400 & 75,000 & $\$ 48,900$ & $\$ 101,400$ \\
\hline 24.0 & 32.0 & 34,800 & 100,000 & $\$ 59,200$ & $\$ 121,100$ \\
\hline 37.0 & 32.0 & 69,400 & 250,000 & $\$ 98,600$ & $\$ 150,900$ \\
\hline 47.0 & 40.0 & 123,100 & 500,000 & $\$ 157,800$ & $\$ 222,400$ \\
\hline 57.0 & 40.0 & 176,400 & 750,000 & $\$ 214,800$ & $\$ 296,800$ \\
\hline 66.0 & 40.0 & 228,000 & $1,000,000$ & $\$ 266,100$ & $\$ 349,700$ \\
\hline 134.0 & 48.0 & 853,600 & $5,000,000$ & $\$ 864,300$ & $\$ 1,054,000$ \\
\hline 175.0 & 56.0 & $2,226,100$ & $10,000,000$ & $\$ 2,040,700$ & $\$ 2,332,000$ \\
\hline
\end{tabular}


Valve Tray Columns

$1^{\text {st }}$ Quarter 1998 dollars

\begin{tabular}{|c|c|c|c|c|c|}
\hline & & $15 \mathrm{psi}$ & & $150 p$ & \\
\hline $\begin{array}{c}\text { Diameter } \\
\text { (ft) }\end{array}$ & $\begin{array}{l}\text { Number } \\
\text { of Trays }\end{array}$ & $\begin{array}{c}\text { Purchased } \\
\text { Equipment } \\
\text { Cost (\$) }\end{array}$ & $\begin{array}{c}\text { Installed } \\
\text { Cost (\$) }\end{array}$ & $\begin{array}{c}\text { Purchased } \\
\text { Equipment } \\
\text { Cost (\$) }\end{array}$ & $\begin{array}{c}\text { Installed } \\
\text { Cost (\$) }\end{array}$ \\
\hline 5 & 2 & $\$ 30,600$ & $\$ 159,500$ & $\$ 35,200$ & $\$ 161,300$ \\
\hline 5 & 6 & $\$ 42,300$ & $\$ 175,700$ & $\$ 50,000$ & $\$ 180,600$ \\
\hline 5 & 10 & $\$ 49,000$ & $\$ 192,100$ & $\$ 57,300$ & $\$ 192,000$ \\
\hline 5 & 14 & $\$ 56,100$ & $\$ 203,400$ & $\$ 67,300$ & $\$ 206,200$ \\
\hline 5 & 20 & $\$ 69,700$ & $\$ 225,900$ & $\$ 84,700$ & $\$ 232,500$ \\
\hline 5 & 26 & $\$ 82,300$ & $\$ 246,200$ & $\$ 95,800$ & $\$ 251,000$ \\
\hline 5 & 34 & $\$ 99,800$ & $\$ 285,800$ & $\$ 118,500$ & $\$ 285,300$ \\
\hline 5 & 40 & $\$ 115,200$ & $\$ 310,300$ & $\$ 134,500$ & $\$ 315,300$ \\
\hline 5 & 46 & $\$ 132,000$ & $\$ 335,200$ & $\$ 145,000$ & $\$ 332,700$ \\
\hline 5 & 52 & $\$ 164,900$ & $\$ 378,000$ & $\$ 185,200$ & $\$ 382,600$ \\
\hline 5 & 60 & $\$ 204,900$ & $\$ 429,700$ & $\$ 226,000$ & $\$ 435,000$ \\
\hline 10 & 2 & $\$ 62,500$ & $\$ 249,000$ & $\$ 89,600$ & $\$ 269,500$ \\
\hline 10 & 6 & $\$ 88,400$ & $\$ 282,100$ & $\$ 122,800$ & $\$ 309,900$ \\
\hline 10 & 10 & $\$ 109,700$ & $\$ 311,100$ & $\$ 151,800$ & $\$ 346,700$ \\
\hline 10 & 14 & $\$ 128,600$ & $\$ 349,700$ & $\$ 180,700$ & $\$ 386,000$ \\
\hline 10 & 20 & $\$ 160,400$ & $\$ 394,800$ & $\$ 220,900$ & $\$ 443,400$ \\
\hline 10 & 26 & $\$ 188,500$ & $\$ 436,200$ & $\$ 254,200$ & $\$ 492,200$ \\
\hline 10 & 34 & $\$ 233,600$ & $\$ 498,700$ & $\$ 312,500$ & $\$ 565,800$ \\
\hline 10 & 40 & $\$ 263,800$ & $\$ 558,700$ & $\$ 356,300$ & $\$ 624,000$ \\
\hline 10 & 46 & $\$ 297,100$ & $\$ 605,000$ & $\$ 391,300$ & $\$ 678,300$ \\
\hline 10 & 52 & $\$ 343,000$ & $\$ 666,100$ & $\$ 450,000$ & $\$ 754,600$ \\
\hline 10 & 60 & $\$ 388,400$ & $\$ 727,700$ & $\$ 501,900$ & $\$ 822,100$ \\
\hline 15 & 2 & $\$ 119,900$ & $\$ 396,200$ & $\$ 221,500$ & $\$ 475,100$ \\
\hline 15 & 6 & $\$ 171,000$ & $\$ 469,300$ & $\$ 293,000$ & $\$ 559,000$ \\
\hline 15 & 10 & $\$ 225,700$ & $\$ 539,500$ & $\$ 364,500$ & $\$ 652,400$ \\
\hline 15 & 14 & $\$ 262,500$ & $\$ 587,100$ & $\$ 425,800$ & $\$ 725,200$ \\
\hline 15 & 20 & $\$ 332,400$ & $\$ 677,700$ & $\$ 522,400$ & $\$ 843,700$ \\
\hline 15 & 26 & $\$ 387,000$ & $\$ 767,500$ & $\$ 600,200$ & $\$ 943,900$ \\
\hline 15 & 34 & $\$ 473,900$ & $\$ 878,600$ & $\$ 722,100$ & $\$ 1,089,500$ \\
\hline 15 & 40 & $\$ 538,600$ & $\$ 958,700$ & $\$ 808,900$ & $\$ 1,191,500$ \\
\hline 15 & 46 & $\$ 620,900$ & $\$ 1,061,600$ & $\$ 907,000$ & $\$ 1,314,300$ \\
\hline 15 & 52 & $\$ 689,200$ & $\$ 1,147,900$ & $\$ 997,700$ & $\$ 1,423,400$ \\
\hline 15 & 60 & $\$ 786,500$ & $\$ 1,269,800$ & $\$ 1,145,800$ & $\$ 1,594,100$ \\
\hline 20 & 2 & $\$ 174,900$ & $\$ 574,900$ & $\$ 402,000$ & $\$ 806,800$ \\
\hline 20 & 6 & $\$ 247,900$ & $\$ 674,400$ & $\$ 517,300$ & $\$ 945,200$ \\
\hline 20 & 10 & $\$ 359,400$ & $\$ 815,300$ & $\$ 605,100$ & $\$ 1,064,600$ \\
\hline 20 & 14 & $\$ 421,000$ & $\$ 892,200$ & $\$ 715,700$ & $\$ 1,190,500$ \\
\hline 20 & 20 & $\$ 508,000$ & $\$ 1,023,200$ & $\$ 857,000$ & $\$ 1,363,200$ \\
\hline 20 & 26 & $\$ 585,300$ & $\$ 1,114,100$ & $\$ 993,600$ & $\$ 1,520,800$ \\
\hline 20 & 34 & $\$ 726,300$ & $\$ 1,285,400$ & $\$ 1,203,000$ & $\$ 1,762,200$ \\
\hline 20 & 40 & $\$ 834,300$ & $\$ 1,421,000$ & $\$ 1,347,900$ & $\$ 1,931,400$ \\
\hline 20 & 46 & $\$ 952,800$ & $\$ 1,560,900$ & $\$ 1,526,400$ & $\$ 2,138,200$ \\
\hline 20 & 52 & $\$ 1,051,100$ & $\$ 1,682,200$ & $\$ 1,669,100$ & $\$ 2,314,600$ \\
\hline 20 & 60 & $\$ 1,195,500$ & $\$ 1,856,100$ & $\$ 1,892,600$ & $\$ 2,568,700$ \\
\hline
\end{tabular}


Sieve Tray Columns

$1^{\text {st }}$ Quarter 1998 dollars

\begin{tabular}{|c|c|c|c|c|c|c|}
\hline & & & $15 \mathrm{ps}$ & & $150 p$ & \\
\hline $\begin{array}{c}\text { Diameter } \\
(\mathrm{ft})\end{array}$ & $\begin{array}{l}\text { Number } \\
\text { of Trays }\end{array}$ & $\begin{array}{c}\text { Tangent/ } \\
\text { Tangent } \\
\text { Height }\end{array}$ & $\begin{array}{c}\text { Purchased } \\
\text { Equipment } \\
\text { Cost (\$) }\end{array}$ & $\begin{array}{c}\text { Installed } \\
\text { Cost (\$) }\end{array}$ & $\begin{array}{c}\text { Purchased } \\
\text { Equipment } \\
\text { Cost (\$) }\end{array}$ & $\begin{array}{l}\text { Installed } \\
\text { Cost (\$) }\end{array}$ \\
\hline 5 & 2 & 17 & $\$ 30,000$ & $\$ 158,900$ & $\$ 34,700$ & $\$ 160,800$ \\
\hline 5 & 6 & 25 & $\$ 41,200$ & $\$ 174,600$ & $\$ 48,900$ & $\$ 179,500$ \\
\hline 5 & 10 & 33 & $\$ 47,500$ & $\$ 190,600$ & $\$ 55,800$ & $\$ 190,500$ \\
\hline 5 & 14 & 41 & $\$ 54,200$ & $\$ 201,400$ & $\$ 65,400$ & $\$ 204,300$ \\
\hline 5 & 20 & 53 & $\$ 67,400$ & $\$ 223,500$ & $\$ 82,300$ & $\$ 230,000$ \\
\hline 5 & 26 & 65 & $\$ 79,500$ & $\$ 243,200$ & $\$ 93,000$ & $\$ 248,100$ \\
\hline 5 & 34 & 81 & $\$ 96,300$ & $\$ 282,200$ & $\$ 115,000$ & $\$ 281,700$ \\
\hline 5 & 40 & 93 & $\$ 111,000$ & $\$ 305,900$ & $\$ 130,300$ & $\$ 310,900$ \\
\hline 5 & 46 & 105 & $\$ 126,800$ & $\$ 329,700$ & $\$ 140,200$ & $\$ 327,700$ \\
\hline 5 & 52 & 117 & $\$ 159,500$ & $\$ 372,400$ & $\$ 179,800$ & $\$ 377,000$ \\
\hline 5 & 60 & 133 & $\$ 203,300$ & $\$ 428,100$ & $\$ 218,900$ & $\$ 427,500$ \\
\hline 10 & 2 & 17 & $\$ 60,600$ & $\$ 247,100$ & $\$ 87,700$ & $\$ 267,600$ \\
\hline 10 & 6 & 25 & $\$ 84,600$ & $\$ 278,200$ & $\$ 119,000$ & $\$ 306,100$ \\
\hline 10 & 10 & 33 & $\$ 104,500$ & $\$ 305,800$ & $\$ 146,500$ & $\$ 341,300$ \\
\hline 10 & 14 & 41 & $\$ 122,100$ & $\$ 343,100$ & $\$ 174,200$ & $\$ 379,400$ \\
\hline 10 & 20 & 53 & $\$ 152,300$ & $\$ 386,500$ & $\$ 212,800$ & $\$ 435,000$ \\
\hline 10 & 26 & 65 & $\$ 178,900$ & $\$ 426,300$ & $\$ 244,700$ & $\$ 482,300$ \\
\hline 10 & 34 & 81 & $\$ 221,100$ & $\$ 485,700$ & $\$ 300,000$ & $\$ 552,800$ \\
\hline 10 & 40 & 93 & $\$ 248,400$ & $\$ 542,700$ & $\$ 341,500$ & $\$ 608,600$ \\
\hline 10 & 46 & 105 & $\$ 280,200$ & $\$ 587,400$ & $\$ 374,400$ & $\$ 661,000$ \\
\hline 10 & 52 & 117 & $\$ 324,600$ & $\$ 647,000$ & $\$ 430,900$ & $\$ 735,100$ \\
\hline 10 & 60 & 133 & $\$ 366,300$ & $\$ 704,700$ & $\$ 479,800$ & $\$ 798,100$ \\
\hline 15 & 2 & 17 & $\$ 115,900$ & $\$ 392,100$ & $\$ 217,600$ & $\$ 471,200$ \\
\hline 15 & 6 & 25 & $\$ 163,200$ & $\$ 461,400$ & $\$ 285,200$ & $\$ 551,100$ \\
\hline 15 & 10 & 33 & $\$ 214,900$ & $\$ 528,600$ & $\$ 353,700$ & $\$ 641,300$ \\
\hline 15 & 14 & 41 & $\$ 249,100$ & $\$ 573,400$ & $\$ 412,300$ & $\$ 711,400$ \\
\hline 15 & 20 & 53 & $\$ 315,600$ & $\$ 660,400$ & $\$ 505,600$ & $\$ 826,600$ \\
\hline 15 & 26 & 65 & $\$ 367,100$ & $\$ 746,900$ & $\$ 580,400$ & $\$ 923,600$ \\
\hline 15 & 34 & 81 & $\$ 446,800$ & $\$ 850,800$ & $\$ 696,200$ & $\$ 1,063,100$ \\
\hline 15 & 40 & 93 & $\$ 509,300$ & $\$ 928,700$ & $\$ 778,400$ & $\$ 1,160,300$ \\
\hline 15 & 46 & 105 & $\$ 585,800$ & $\$ 1,025,700$ & $\$ 871,800$ & $\$ 1,278,100$ \\
\hline 15 & 52 & 117 & $\$ 645,700$ & $\$ 1,103,400$ & $\$ 958,000$ & $\$ 1,382,600$ \\
\hline 15 & 60 & 133 & $\$ 739,400$ & $\$ 1,221,700$ & $\$ 1,100,000$ & $\$ 1,546,900$ \\
\hline 20 & 2 & 17 & $\$ 168,200$ & $\$ 568,100$ & $\$ 395,400$ & $\$ 800,100$ \\
\hline 20 & 6 & 25 & $\$ 234,600$ & $\$ 661,000$ & $\$ 504,000$ & $\$ 931,700$ \\
\hline 20 & 10 & 33 & $\$ 341,200$ & $\$ 796,700$ & $\$ 586,800$ & $\$ 1,046,100$ \\
\hline 20 & 14 & 41 & $\$ 398,500$ & $\$ 869,100$ & $\$ 693,100$ & $\$ 1,167,600$ \\
\hline 20 & 20 & 53 & $\$ 479,700$ & $\$ 994,300$ & $\$ 828,800$ & $\$ 1,334,500$ \\
\hline 20 & 26 & 65 & $\$ 551,900$ & $\$ 1,080,000$ & $\$ 960,300$ & $\$ 1,486,500$ \\
\hline 20 & 34 & 81 & $\$ 681,100$ & $\$ 1,239,200$ & $\$ 1,159,400$ & $\$ 1,717,400$ \\
\hline 20 & 40 & 93 & $\$ 781,300$ & $\$ 1,365,200$ & $\$ 1,296,600$ & $\$ 1,876,900$ \\
\hline 20 & 46 & 105 & $\$ 892,200$ & $\$ 1,498,500$ & $\$ 1,467,400$ & $\$ 2,075,600$ \\
\hline 20 & 52 & 117 & $\$ 988,200$ & $\$ 1,624,000$ & $\$ 1,602,400$ & $\$ 2,246,100$ \\
\hline 20 & 60 & 133 & $\$ 1,120,200$ & $\$ 1,778,700$ & $\$ 1,815,600$ & $\$ 2,489,600$ \\
\hline
\end{tabular}


Packed Columns

$1^{\text {st }}$ Quarter 1998 dollars

\begin{tabular}{|c|c|c|c|c|c|c|c|}
\hline & & & & $15 p$ & sig & 150 & sig \\
\hline $\begin{array}{c}\text { Diameter } \\
\text { (Feet) }\end{array}$ & $\begin{array}{c}\text { Tangent/ } \\
\text { Tangent } \\
\text { Height } \\
\text { (Feet) }\end{array}$ & $\begin{array}{c}\text { Packed } \\
\text { Height } \\
\text { (Feet) }\end{array}$ & $\begin{array}{c}\text { Number } \\
\text { of } \\
\text { Sections }\end{array}$ & \begin{tabular}{|c|} 
Purchased \\
Equipment \\
Cost $(\$)$
\end{tabular} & $\begin{array}{c}\text { Installed } \\
\text { Cost (\$) }\end{array}$ & $\begin{array}{c}\text { Purchased } \\
\text { Equipment } \\
\text { Cost (\$) }\end{array}$ & $\begin{array}{c}\text { Installed } \\
\text { Cost (\$) }\end{array}$ \\
\hline 1 & 10 & 8 & 1 & $\$ 6,700$ & $\$ 64,000$ & $\$ 6,600$ & $\$ 62,000$ \\
\hline 1 & 20 & 18 & 3 & $\$ 8,700$ & $\$ 73,400$ & $\$ 9,000$ & $\$ 67,800$ \\
\hline 1.5 & 10 & 8 & 1 & $\$ 10,300$ & $\$ 75,500$ & $\$ 11,300$ & $\$ 69,800$ \\
\hline 1.5 & 20 & 18 & 2 & $\$ 13,900$ & $\$ 83,000$ & $\$ 15,400$ & $\$ 77,600$ \\
\hline 1.5 & 30 & 28 & 3 & $\$ 16,600$ & $\$ 89,700$ & $\$ 18,700$ & $\$ 84,800$ \\
\hline 2 & 10 & 8 & 1 & $\$ 12,900$ & $\$ 82,800$ & $\$ 13,900$ & $\$ 76,500$ \\
\hline 2 & 20 & 18 & 2 & $\$ 16,900$ & $\$ 90,900$ & $\$ 18,500$ & $\$ 85,000$ \\
\hline 2 & 30 & 28 & 2 & $\$ 18,600$ & $\$ 97,000$ & $\$ 20,100$ & $\$ 90,900$ \\
\hline 2 & 40 & 38 & 3 & $\$ 21,500$ & $\$ 105,500$ & $\$ 23,600$ & $\$ 101,400$ \\
\hline 2.5 & 10 & 8 & 1 & $\$ 14,700$ & $\$ 92,200$ & $\$ 15,400$ & $\$ 82,400$ \\
\hline 2.5 & 20 & 18 & 1 & $\$ 16,700$ & $\$ 98,700$ & $\$ 17,600$ & $\$ 89,000$ \\
\hline 2.5 & 30 & 28 & 2 & $\$ 22,400$ & $\$ 112,000$ & $\$ 23,800$ & $\$ 104,200$ \\
\hline 2.5 & 40 & 38 & 2 & $\$ 23,200$ & $\$ 116,000$ & $\$ 24,600$ & $\$ 108,000$ \\
\hline 2.5 & 50 & 48 & 3 & $\$ 30,000$ & $\$ 127,800$ & $\$ 31,800$ & $\$ 119,800$ \\
\hline 3 & 10 & 8 & 1 & $\$ 16,200$ & $\$ 98,700$ & $\$ 17,200$ & $\$ 89,400$ \\
\hline 3 & 20 & 18 & 1 & $\$ 21,900$ & $\$ 110,800$ & $\$ 23,500$ & $\$ 101,900$ \\
\hline 3 & 30 & 28 & 2 & $\$ 24,300$ & $\$ 119,700$ & $\$ 25,900$ & $\$ 112,100$ \\
\hline 3 & 40 & 38 & 2 & $\$ 26,500$ & $\$ 125,300$ & $\$ 29,200$ & $\$ 118,500$ \\
\hline 3 & 50 & 48 & 3 & $\$ 31,200$ & $\$ 135,400$ & $\$ 34,700$ & $\$ 129,500$ \\
\hline 3 & 60 & 58 & 3 & $\$ 35,400$ & $\$ 147,400$ & $\$ 37,500$ & $\$ 135,900$ \\
\hline 3.5 & 10 & 8 & 1 & $\$ 20,600$ & $\$ 112,300$ & $\$ 23,100$ & $\$ 100,000$ \\
\hline 3.5 & 20 & 18 & 1 & $\$ 26,400$ & $\$ 125,000$ & $\$ 30,600$ & $\$ 118,200$ \\
\hline 3.5 & 30 & 28 & 2 & $\$ 30,400$ & $\$ 135,800$ & $\$ 35,000$ & $\$ 126,300$ \\
\hline 3.5 & 40 & 38 & 2 & $\$ 31,500$ & $\$ 140,800$ & $\$ 36,300$ & $\$ 131,300$ \\
\hline 3.5 & 50 & 48 & 3 & $\$ 38,700$ & $\$ 157,600$ & $\$ 45,000$ & $\$ 145,700$ \\
\hline 3.5 & 60 & 58 & 3 & $\$ 43,400$ & $\$ 166,600$ & $\$ 48,000$ & $\$ 152,500$ \\
\hline 3.5 & 70 & 68 & 4 & $\$ 48,400$ & $\$ 178,500$ & $\$ 57,600$ & $\$ 168,000$ \\
\hline
\end{tabular}


Shell and Tube Heat Exchangers

$1^{\text {st }}$ Quarter 1998 dollars

\begin{tabular}{|c|r|r|}
\hline $\begin{array}{c}\text { Surface Area, } \\
\text { (Square feet) }\end{array}$ & $\begin{array}{r}\text { Purchased } \\
\text { Equipment Cost } \\
(\$)\end{array}$ & $\begin{array}{c}\text { Installed Cost } \\
\mathbf{( \$ )}\end{array}$ \\
\hline 100 & $\$ 13,200$ & $\$ 48,300$ \\
\hline 200 & $\$ 13,600$ & $\$ 55,800$ \\
\hline 300 & $\$ 14,500$ & $\$ 57,300$ \\
\hline 400 & $\$ 16,100$ & $\$ 59,100$ \\
\hline 500 & $\$ 16,200$ & $\$ 68,000$ \\
\hline 600 & $\$ 16,600$ & $\$ 68,400$ \\
\hline 700 & $\$ 18,000$ & $\$ 70,000$ \\
\hline 800 & $\$ 18,400$ & $\$ 70,400$ \\
\hline 900 & $\$ 20,300$ & $\$ 72,600$ \\
\hline 1000 & $\$ 20,800$ & $\$ 73,100$ \\
\hline 2000 & $\$ 31,900$ & $\$ 95,800$ \\
\hline 3000 & $\$ 44,700$ & $\$ 109,600$ \\
\hline 4000 & $\$ 53,900$ & $\$ 132,900$ \\
\hline 5000 & $\$ 62,100$ & $\$ 141,800$ \\
\hline 6000 & $\$ 70,800$ & $\$ 151,100$ \\
\hline 7000 & $\$ 99,600$ & $\$ 203,500$ \\
\hline 8000 & $\$ 107,900$ & $\$ 212,400$ \\
\hline 9000 & $\$ 117,100$ & $\$ 222,100$ \\
\hline 10000 & $\$ 124,200$ & $\$ 229,800$ \\
\hline 15000 & $\$ 186,300$ & $\$ 321,500$ \\
\hline 20000 & $\$ 248,400$ & $\$ 427,000$ \\
\hline 30000 & $\$ 354,000$ & $\$ 573,900$ \\
\hline 40000 & $\$ 479,100$ & $\$ 767,500$ \\
\hline 50000 & $\$ 582,500$ & $\$ 953,000$ \\
\hline 60000 & $\$ 708,300$ & $\$ 1,106,600$ \\
\hline 70000 & $\$ 839,000$ & $\$ 1,425,600$ \\
\hline
\end{tabular}


Air Cooler

$1^{\text {st }}$ Quarter 1998 dollars

\begin{tabular}{|c|r|r|}
\hline $\begin{array}{c}\text { Surface Area, } \\
\text { (Square feet) }\end{array}$ & $\begin{array}{r}\text { Purchased } \\
\text { Equipment Cost } \\
\mathbf{( \$ )}\end{array}$ & $\begin{array}{c}\text { Installed Cost } \\
\mathbf{( \$ )}\end{array}$ \\
\hline 100 & $\$ 21,300$ & $\$ 47,600$ \\
\hline 200 & $\$ 24,100$ & $\$ 51,800$ \\
\hline 300 & $\$ 26,100$ & $\$ 54,800$ \\
\hline 400 & $\$ 29,100$ & $\$ 58,100$ \\
\hline 500 & $\$ 30,900$ & $\$ 59,900$ \\
\hline 600 & $\$ 33,000$ & $\$ 62,000$ \\
\hline 700 & $\$ 36,000$ & $\$ 65,300$ \\
\hline 800 & $\$ 38,100$ & $\$ 67,400$ \\
\hline 900 & $\$ 40,300$ & $\$ 69,900$ \\
\hline 1,000 & $\$ 42,000$ & $\$ 71,600$ \\
\hline 2,000 & $\$ 60,800$ & $\$ 94,100$ \\
\hline 4,000 & $\$ 96,900$ & $\$ 144,700$ \\
\hline 6,000 & $\$ 135,400$ & $\$ 184,700$ \\
\hline 8,000 & $\$ 179,100$ & $\$ 239,000$ \\
\hline 10,000 & $\$ 217,300$ & $\$ 278,200$ \\
\hline & & \\
\hline
\end{tabular}

Spiral Plate Heat Exchanger

$1^{\text {st }}$ Quarter 1998 dollars

\begin{tabular}{|c|r|r|}
\hline $\begin{array}{c}\text { Heat Transfer } \\
\text { Area, } \\
\text { (Square feet) }\end{array}$ & $\begin{array}{c}\text { Purchased } \\
\text { Equipment Cost } \\
\mathbf{( \$ )}\end{array}$ & $\begin{array}{c}\text { Installed Cost } \\
\mathbf{( \$ )}\end{array}$ \\
\hline 40 & $\$ 6,700$ & $\$ 19,200$ \\
\hline 100 & $\$ 9,100$ & $\$ 25,100$ \\
\hline 200 & $\$ 13,200$ & $\$ 34,000$ \\
\hline 300 & $\$ 21,100$ & $\$ 49,400$ \\
\hline 400 & $\$ 25,500$ & $\$ 57,400$ \\
\hline 500 & $\$ 29,900$ & $\$ 65,000$ \\
\hline 600 & $\$ 34,400$ & $\$ 72,400$ \\
\hline 700 & $\$ 42,600$ & $\$ 85,300$ \\
\hline 800 & $\$ 35,500$ & $\$ 74,200$ \\
\hline 900 & $\$ 40,000$ & $\$ 81,300$ \\
\hline 1,000 & $\$ 44,700$ & $\$ 88,500$ \\
\hline 1,100 & $\$ 49,600$ & $\$ 95,700$ \\
\hline 1,200 & $\$ 54,700$ & $\$ 102,900$ \\
\hline 1,300 & $\$ 60,100$ & $\$ 110,400$ \\
\hline
\end{tabular}


Furnace

$1^{\text {st }}$ Quarter 1998 dollars

\begin{tabular}{|c|r|r|}
\hline $\begin{array}{c}\text { Heat Duty } \\
\text { (MMBTU per } \\
\text { hour) }\end{array}$ & $\begin{array}{c}\text { Purchased } \\
\text { Equipment Cost } \\
\mathbf{( \$ )}\end{array}$ & $\begin{array}{c}\text { Installed Cost } \\
\mathbf{( \$ )}\end{array}$ \\
\hline 2 & $\$ 124,600$ & $\$ 96,300$ \\
\hline 10 & $\$ 263,100$ & $\$ 355,100$ \\
\hline 25 & $\$ 399,000$ & $\$ 518,600$ \\
\hline 50 & $\$ 625,400$ & $\$ 771,100$ \\
\hline 100 & $\$ 1,081,500$ & $\$ 1,272,800$ \\
\hline 200 & $\$ 1,868,900$ & $\$ 2,641,500$ \\
\hline 300 & $\$ 2,573,100$ & $\$ 3,534,400$ \\
\hline 400 & $\$ 3,228,000$ & $\$ 4,354,800$ \\
\hline 500 & $\$ 3,848,400$ & $\$ 5,126,000$ \\
\hline
\end{tabular}

Cooling Tower

$1^{\text {st }}$ Quarter 1998 dollars

\begin{tabular}{|r|r|r|}
\hline $\begin{array}{c}\text { Water Rate } \\
\text { (Gallons/ } \\
\text { minute) }\end{array}$ & $\begin{array}{c}\text { Purchased } \\
\text { Equipment Cost } \\
\mathbf{( \$ )}\end{array}$ & $\begin{array}{c}\text { Installed Cost } \\
\mathbf{( \$ )}\end{array}$ \\
\hline 150 & $\$ 4,000$ & $\$ 60,200$ \\
\hline 300 & $\$ 6,500$ & $\$ 65,000$ \\
\hline 600 & $\$ 11,400$ & $\$ 70,500$ \\
\hline 1,000 & $\$ 18,000$ & $\$ 81,700$ \\
\hline 2,000 & $\$ 34,400$ & $\$ 106,100$ \\
\hline 3,000 & $\$ 50,900$ & $\$ 134,200$ \\
\hline 4,000 & $\$ 67,100$ & $\$ 158,800$ \\
\hline 5,000 & $\$ 83,200$ & $\$ 180,400$ \\
\hline 6,000 & $\$ 99,200$ & $\$ 211,100$ \\
\hline
\end{tabular}


Package Steam Boiler

$1^{\text {st }}$ Quarter 1998 dollars

\begin{tabular}{|c|r|r|}
\hline $\begin{array}{c}\text { Capacity } \\
\text { (Pound per } \\
\text { hour) }\end{array}$ & $\begin{array}{c}\text { Purchased } \\
\text { Equipment Cost } \\
\mathbf{( \$ )}\end{array}$ & $\begin{array}{c}\text { Installed Cost } \\
\mathbf{( \$ )}\end{array}$ \\
\hline 10,000 & $\$ 91,700$ & $\$ 283,100$ \\
\hline 25,000 & $\$ 148,100$ & $\$ 368,900$ \\
\hline 50,000 & $\$ 212,700$ & $\$ 468,900$ \\
\hline 100,000 & $\$ 305,700$ & $\$ 607,300$ \\
\hline 150,000 & $\$ 439,400$ & $\$ 783,600$ \\
\hline 200,000 & $\$ 568,400$ & $\$ 920,600$ \\
\hline 250,000 & $\$ 694,000$ & $\$ 1,109,100$ \\
\hline 300,000 & $\$ 816,900$ & $\$ 1,238,600$ \\
\hline
\end{tabular}

Evaporator

$1^{\text {st }}$ Quarter 1998 dollars

\begin{tabular}{|r|r|r|r|r|}
\hline $\begin{array}{c}\text { Area } \\
\text { (Square } \\
\text { feet) }\end{array}$ & $\begin{array}{c}\text { Purchased } \\
\text { Equipment } \\
\text { Cost } \\
\mathbf{( \$ )}\end{array}$ & $\begin{array}{c}\text { Installed } \\
\text { Cost } \\
\mathbf{( \$ )}\end{array}$ & $\begin{array}{c}\text { Purchased } \\
\text { Equipment } \\
\text { Cost } \\
\mathbf{( \$ )}\end{array}$ & $\begin{array}{c}\text { Installed } \\
\text { Cost } \\
\mathbf{( \$ )}\end{array}$ \\
\hline 100 & $\$ 62,600$ & $\$ 120,800$ & $\$ 34,500$ & $\$ 73,300$ \\
\hline 500 & $\$ 151,600$ & $\$ 273,500$ & $\$ 81,100$ & $\$ 161,300$ \\
\hline 1,000 & $\$ 221,900$ & $\$ 388,400$ & $\$ 117,100$ & $\$ 226,300$ \\
\hline 2,000 & $\$ 324,700$ & $\$ 555,200$ & $\$ 169,000$ & $\$ 317,100$ \\
\hline 3,000 & $\$ 405,700$ & $\$ 689,100$ & $\$ 209,500$ & $\$ 386,300$ \\
\hline 4,000 & $\$ 475,200$ & $\$ 803,300$ & $\$ 244,100$ & $\$ 444,300$ \\
\hline 5,000 & $\$ 537,100$ & $\$ 904,700$ & $\$ 274,400$ & $\$ 496,800$ \\
\hline 6,000 & $\$ 593,700$ & $\$ 997,000$ & $\$ 302,600$ & $\$ 545,600$ \\
\hline 7,000 & & & $\$ 328,300$ & $\$ 590,500$ \\
\hline 8,000 & & & $\$ 352,400$ & $\$ 632,400$ \\
\hline 9,000 & & & $\$ 375,100$ & $\$ 671,900$ \\
\hline 10,000 & & & $\$ 396,600$ & $\$ 709,200$ \\
\hline
\end{tabular}




\section{Crusher}

$1^{\text {st }}$ Quarter 1998 dollars

\begin{tabular}{|c|c|c|c|}
\hline $\begin{array}{c}\text { Diameter } \\
\text { (Inches) }\end{array}$ & $\begin{array}{l}\text { Driver Power } \\
\text { (Horsepower) }\end{array}$ & $\begin{array}{c}\text { Purchased } \\
\text { Equipment } \\
\text { Cost } \\
\text { (\$) }\end{array}$ & $\begin{array}{c}\text { Installed Cost } \\
\text { (\$) }\end{array}$ \\
\hline \multicolumn{4}{|c|}{ Gyratory Crusher } \\
\hline 20 & 40 & $\$ 29,300$ & $\$ 52,400$ \\
\hline 40 & 150 & $\$ 253,600$ & $\$ 294,400$ \\
\hline 60 & 350 & $\$ 698,200$ & $\$ 787,200$ \\
\hline 80 & 600 & $\$ 1,400,900$ & $\$ 1,553,600$ \\
\hline 100 & 900 & $\$ 2,415,500$ & $\$ 2,666,100$ \\
\hline 120 & 1250 & $\$ 3,778,800$ & $\$ 4,171,200$ \\
\hline \multicolumn{4}{|c|}{ Rotary Crusher } \\
\hline & 2 & $\$ 2,300$ & $\$ 5,200$ \\
\hline & 4 & $\$ 3,700$ & $\$ 6,800$ \\
\hline & 8 & $\$ 6,100$ & $\$ 9,500$ \\
\hline & 12 & $\$ 8,100$ & $\$ 11,800$ \\
\hline & 16 & $\$ 9,900$ & $\$ 13,900$ \\
\hline & 20 & $\$ 11,600$ & $\$ 15,800$ \\
\hline & 25 & $\$ 13,600$ & $\$ 18,100$ \\
\hline \multicolumn{4}{|c|}{ Ring Granulator } \\
\hline & 75 & $\$ 23,400$ & $\$ 28,100$ \\
\hline & 125 & $\$ 50,700$ & $\$ 58,000$ \\
\hline & 250 & $\$ 75,900$ & $\$ 85,900$ \\
\hline & 600 & $\$ 197,400$ & $\$ 218,700$ \\
\hline & 1000 & $\$ 303,300$ & $\$ 335,600$ \\
\hline & 1250 & $\$ 346,400$ & $\$ 382,200$ \\
\hline
\end{tabular}


Mill

$1^{\text {st }}$ Quarter 1998 dollars

\begin{tabular}{|c|c|r|r|}
\hline $\begin{array}{c}\text { Diameterl } \\
\text { Length } \\
\text { (Inches) }\end{array}$ & $\begin{array}{c}\text { Driver Power } \\
\text { (Horsepower) }\end{array}$ & $\begin{array}{c}\text { Purchased } \\
\text { Equipment Cost } \\
\mathbf{( \$ )}\end{array}$ & $\begin{array}{c}\text { Installed Cost } \\
\mathbf{( \$ )}\end{array}$ \\
\hline \multicolumn{2}{|c|}{ Ball Mill } & $\$ 25,100$ & $\$ 62,900$ \\
\hline $3 / 3$ & 7.5 & $\$ 57,500$ & $\$ 97,900$ \\
\hline $4 / 4$ & 20 & $\$ 109,100$ & $\$ 153,500$ \\
\hline $5 / 5$ & 50 & $\$ 182,900$ & $\$ 234,400$ \\
\hline $6 / 6$ & 100 & $\$ 255,600$ & $\$ 311,700$ \\
\hline & 200 & $\$ 411,300$ & $\$ 478,500$ \\
\hline & 300 & $\$ 585,200$ & $\$ 573,100$ \\
\hline & 400 & & $\$ 673,100$ \\
\hline & 450 & $\$ 107,500$ & $\$ 13,900$ \\
\hline Roller Mill & 30 & $\$ 164,200$ & $\$ 197,000$ \\
\hline & 75 & $\$ 195,800$ & $\$ 233,100$ \\
\hline & 150 & $\$ 250,900$ & $\$ 296,100$ \\
\hline & 200 & $\$ 275,700$ & $\$ 324,400$ \\
\hline & 250 & $\$ 299,100$ & $\$ 351,000$ \\
\hline
\end{tabular}


Dryers

$1^{\text {st }}$ Quarter 1998 dollars

\begin{tabular}{|c|r|r|r|}
\hline $\begin{array}{c}\text { Area } \\
\text { (Square } \\
\text { feet) }\end{array}$ & $\begin{array}{r}\text { Driver Power } \\
\text { (Horsepower) }\end{array}$ & $\begin{array}{r}\text { Purchased } \\
\text { Equipment Cost } \\
\mathbf{( \$ )}\end{array}$ & $\begin{array}{c}\text { Installed Cost } \\
\mathbf{( \$ )}\end{array}$ \\
\hline \multicolumn{2}{|c|}{ Direct Contact Rotary Dryer } & \\
\hline 100 & & $\$ 26,500$ & $\$ 42,400$ \\
\hline 400 & & $\$ 19,500$ & $\$ 142,800$ \\
\hline 800 & & $\$ 283,600$ & $\$ 264,800$ \\
\hline 1200 & & $\$ 373,100$ & $\$ 380,800$ \\
\hline 1600 & & $\$ 461,500$ & $\$ 603,400$ \\
\hline 2000 & & $\$ 53,900$ & \\
\hline \multicolumn{2}{|c|}{ Single Atmospheric Drum Dryer } & $\$ 73,800$ \\
\hline 10 & 5 & $\$ 125,800$ & $\$ 162,900$ \\
\hline 40 & 10 & $\$ 192,300$ & $\$ 243,800$ \\
\hline 80 & 15 & $\$ 246,500$ & $\$ 309,100$ \\
\hline 120 & 20 & $\$ 293,900$ & $\$ 365,900$ \\
\hline 160 & 20 & $\$ 337,100$ & $\$ 417,400$ \\
\hline 200 & 25 & $\$ 6,400$ & $\$ 10,900$ \\
\hline \multicolumn{2}{|c|}{ Atmospheric Tray Batch Dryer } & $\$ 13,900$ \\
\hline 30 & & $\$ 8,400$ & $\$ 16,000$ \\
\hline 60 & & $\$ 9,800$ & $\$ 17,700$ \\
\hline 90 & & $\$ 10,900$ & $\$ 19,200$ \\
\hline 120 & & $\$ 1,900$ & $\$ 20,500$ \\
\hline 150 & & $\$ 12,800$ & \\
\hline 180 & & & \\
\hline 200 & & &
\end{tabular}


Centrifuge

$1^{\text {st }}$ Quarter 1998 dollars

\begin{tabular}{|c|c|c|c|}
\hline $\begin{array}{l}\text { Screen } \\
\text { Diameter } \\
\text { (Inches) }\end{array}$ & $\begin{array}{l}\text { Driver Power } \\
\text { (Horsepower) }\end{array}$ & $\begin{array}{c}\text { Purchased } \\
\text { Equipment } \\
\text { Cost (\$) }\end{array}$ & $\begin{array}{l}\text { Installed Cost } \\
\text { (\$) }\end{array}$ \\
\hline \multicolumn{4}{|c|}{ Batch Bottom-Suspended Filtering Centrifuge } \\
\hline 20 & 1.5 & $\$ 10,100$ & $\$ 21,500$ \\
\hline 25 & 2 & $\$ 11,900$ & $\$ 23,500$ \\
\hline 30 & 3 & $\$ 13,600$ & $\$ 25,500$ \\
\hline 35 & 5 & $\$ 15,300$ & $\$ 27,400$ \\
\hline 40 & 7.5 & $\$ 16,900$ & $\$ 29,300$ \\
\hline 45 & 10 & $\$ 18,400$ & $\$ 31,100$ \\
\hline 48 & 10 & $\$ 19,300$ & $\$ 32,200$ \\
\hline \multicolumn{4}{|c|}{ Batch Top-Suspended Filtering Centrifuge } \\
\hline 20 & 1.5 & $\$ 12,000$ & $\$ 23,400$ \\
\hline 25 & 2 & $\$ 16,000$ & $\$ 27,700$ \\
\hline 30 & 3 & $\$ 20,200$ & $\$ 32,300$ \\
\hline 35 & 5 & $\$ 24,700$ & $\$ 37,100$ \\
\hline 40 & 7.5 & $\$ 29,300$ & $\$ 42,100$ \\
\hline 45 & 10 & $\$ 34,100$ & $\$ 47,300$ \\
\hline 50 & 15 & $\$ 39,100$ & $\$ 52,800$ \\
\hline \multicolumn{4}{|c|}{ Continuous Filtration Vibratory Centrifuge } \\
\hline 48 & 30 & $\$ 58,600$ & $\$ 91,900$ \\
\hline 50 & 40 & $\$ 66,700$ & $\$ 100,900$ \\
\hline 52 & 50 & $\$ 75,500$ & $\$ 113,000$ \\
\hline 54 & 60 & $\$ 85,000$ & $\$ 124,000$ \\
\hline 56 & 75 & $\$ 95,400$ & $\$ 135,800$ \\
\hline \multicolumn{4}{|c|}{ Reciprocating Conveyor, w/Continuous Filtering Centrifuge } \\
\hline 15 & & $\$ 112,900$ & $\$ 140,500$ \\
\hline 25 & & $\$ 175,200$ & $\$ 213,200$ \\
\hline 35 & & $\$ 246,100$ & $\$ 295,100$ \\
\hline 45 & & $\$ 317,200$ & $\$ 376,200$ \\
\hline 50 & & $\$ 352,900$ & $\$ 416,800$ \\
\hline
\end{tabular}




\section{Filter}

$1^{\text {st }}$ Quarter 1998 dollars

\begin{tabular}{|c|c|c|c|c|}
\hline \begin{tabular}{|c|} 
Flow Rate \\
(Gallons per \\
minute)
\end{tabular} & $\begin{array}{c}\text { Frame } \\
\text { Capacity } \\
\text { (Cubic } \\
\text { feet) }\end{array}$ & $\begin{array}{c}\text { Surface } \\
\text { Area } \\
\text { (Square } \\
\text { feet) }\end{array}$ & $\begin{array}{c}\text { Purchased } \\
\text { Equipment } \\
\text { Cost } \\
\text { (\$) } \\
\end{array}$ & $\begin{array}{c}\text { Installed } \\
\text { Cost } \\
\text { (\$) }\end{array}$ \\
\hline \multicolumn{5}{|c|}{ Cartridge Filter } \\
\hline 30 & & & $\$ 1,100$ & $\$ 5,200$ \\
\hline 100 & & & $\$ 1,700$ & $\$ 6,800$ \\
\hline 300 & & & $\$ 2,400$ & $\$ 8,300$ \\
\hline 600 & & & $\$ 4,200$ & $\$ 10,300$ \\
\hline 900 & & & $\$ 5,800$ & $\$ 13,500$ \\
\hline 1200 & & & $\$ 7,300$ & $\$ 15,200$ \\
\hline \multicolumn{5}{|c|}{ Automatic Plate and Frame } \\
\hline & 10 & & $\$ 100,200$ & $\$ 145,500$ \\
\hline & 20 & & $\$ 114,200$ & $\$ 160,400$ \\
\hline & 30 & & $\$ 123,300$ & $\$ 170,100$ \\
\hline & 40 & & $\$ 130,200$ & $\$ 177,500$ \\
\hline & 50 & & $\$ 135,900$ & $\$ 183,600$ \\
\hline \multicolumn{5}{|c|}{ Tubular Fabric Filter } \\
\hline 100 & & & $\$ 5,500$ & $\$ 13,000$ \\
\hline 500 & & & $\$ 15,700$ & $\$ 27,100$ \\
\hline 1000 & & & $\$ 24,700$ & $\$ 39,900$ \\
\hline 1500 & & & $\$ 32,200$ & $\$ 51,200$ \\
\hline 2000 & & & $\$ 38,800$ & $\$ 59,500$ \\
\hline 2500 & & & $\$ 44,900$ & $\$ 69,200$ \\
\hline 3000 & & & $\$ 50,600$ & $\$ 76,400$ \\
\hline 3400 & & & $\$ 54,900$ & $\$ 81,700$ \\
\hline \multicolumn{5}{|c|}{ Drum Filter } \\
\hline & & 100 & $\$ 63,400$ & $\$ 104,200$ \\
\hline & & 250 & $\$ 87,700$ & $\$ 134,400$ \\
\hline & & 500 & $\$ 120,200$ & $\$ 175,400$ \\
\hline & & 750 & $\$ 145,000$ & $\$ 205,200$ \\
\hline & & 1000 & $\$ 168,900$ & $\$ 237,400$ \\
\hline & & 1500 & $\$ 192,900$ & $\$ 275,700$ \\
\hline & & 2000 & $\$ 208,300$ & $\$ 298,900$ \\
\hline
\end{tabular}




\section{Agitators}

$1^{\text {st }}$ Quarter 1998 dollars

\begin{tabular}{|c|r|r|}
\hline $\begin{array}{c}\text { Driver Power } \\
\text { (Horsepower) }\end{array}$ & $\begin{array}{c}\text { Purchased } \\
\text { Equipment Cost } \\
\text { (\$) }\end{array}$ & $\begin{array}{c}\text { Installed Cost } \\
\mathbf{( \$ )}\end{array}$ \\
\hline 2 & $\$ 7,700$ & $\$ 9,500$ \\
\hline 10 & $\$ 13,900$ & $\$ 15,900$ \\
\hline 25 & $\$ 19,500$ & $\$ 21,600$ \\
\hline 50 & $\$ 35,400$ & $\$ 37,700$ \\
\hline 75 & $\$ 50,200$ & $\$ 52,700$ \\
\hline 100 & $\$ 64,300$ & $\$ 67,000$ \\
\hline
\end{tabular}

\section{Rotary Pump}

$1^{\text {st }}$ Quarter 1998 dollars

\begin{tabular}{|c|r|r|}
\hline $\begin{array}{c}\text { Capacity } \\
\text { (Gallons/ } \\
\text { minute) }\end{array}$ & $\begin{array}{c}\text { Purchased } \\
\text { Equipment Cost } \\
\mathbf{( \$ )}\end{array}$ & $\begin{array}{c}\text { Installed Cost } \\
\mathbf{( \$ )}\end{array}$ \\
\hline 10 & $\$ 1,500$ & $\$ 9,000$ \\
\hline 50 & $\$ 2,100$ & $\$ 10,900$ \\
\hline 100 & $\$ 2,400$ & $\$ 12,600$ \\
\hline 150 & $\$ 3,000$ & $\$ 13,200$ \\
\hline 200 & $\$ 3,400$ & $\$ 13,700$ \\
\hline 250 & $\$ 4,100$ & $\$ 16,000$ \\
\hline 300 & $\$ 4,400$ & $\$ 16,300$ \\
\hline 400 & $\$ 5,300$ & $\$ 17,300$ \\
\hline 500 & $\$ 7,000$ & $\$ 19,200$ \\
\hline 600 & $\$ 8,700$ & $\$ 21,000$ \\
\hline 700 & $\$ 10,700$ & $\$ 25,700$ \\
\hline 750 & $\$ 11,600$ & $\$ 26,600$ \\
\hline
\end{tabular}


Inline Pump

$1^{\text {st }}$ Quarter 1998 dollars

\begin{tabular}{|c|r|r|}
\hline $\begin{array}{c}\text { Capacity } \\
\text { (Gallons/ } \\
\text { minute) }\end{array}$ & $\begin{array}{c}\text { Purchased } \\
\text { Equipment Cost } \\
\text { (\$) }\end{array}$ & $\begin{array}{c}\text { Installed Cost } \\
\mathbf{( \$ )}\end{array}$ \\
\hline 10 & $\$ 1,500$ & $\$ 9,000$ \\
\hline 50 & $\$ 2,100$ & $\$ 10,900$ \\
\hline 100 & $\$ 2,400$ & $\$ 12,600$ \\
\hline 150 & $\$ 3,000$ & $\$ 13,200$ \\
\hline 200 & $\$ 3,400$ & $\$ 13,700$ \\
\hline 250 & $\$ 4,100$ & $\$ 16,000$ \\
\hline 300 & $\$ 4,400$ & $\$ 16,300$ \\
\hline 400 & $\$ 5,300$ & $\$ 17,300$ \\
\hline 500 & $\$ 7,000$ & $\$ 19,200$ \\
\hline 600 & $\$ 8,700$ & $\$ 21,000$ \\
\hline 700 & $\$ 10,700$ & $\$ 25,700$ \\
\hline 750 & $\$ 11,600$ & $\$ 26,600$ \\
\hline
\end{tabular}

Centrifugal Pump

$1^{\text {st }}$ Quarter 1998 dollars

\begin{tabular}{|c|r|r|}
\hline $\begin{array}{c}\text { Capacity } \\
\text { (Gallons/ } \\
\text { minute) }\end{array}$ & $\begin{array}{c}\text { Purchased } \\
\text { Equipment Cost } \\
\mathbf{( \$ )}\end{array}$ & $\begin{array}{c}\text { Installed Cost } \\
\mathbf{( \$ )}\end{array}$ \\
\hline 100 & $\$ 3,400$ & $\$ 22,800$ \\
\hline 200 & $\$ 4,100$ & $\$ 23,800$ \\
\hline 300 & $\$ 4,700$ & $\$ 27,700$ \\
\hline 400 & $\$ 5,300$ & $\$ 28,500$ \\
\hline 500 & $\$ 5,800$ & $\$ 29,000$ \\
\hline 1,000 & $\$ 8,700$ & $\$ 37,500$ \\
\hline 2,000 & $\$ 10,200$ & $\$ 44,800$ \\
\hline 3,000 & $\$ 15,200$ & $\$ 58,100$ \\
\hline 4,000 & $\$ 19,500$ & $\$ 72,300$ \\
\hline 5,000 & $\$ 23,800$ & $\$ 77,100$ \\
\hline 6,000 & $\$ 28,400$ & $\$ 93,400$ \\
\hline 7,000 & $\$ 37,800$ & $\$ 103,000$ \\
\hline 8,000 & $\$ 41,300$ & $\$ 119,700$ \\
\hline 9,000 & $\$ 47,300$ & $\$ 126,200$ \\
\hline 10,000 & $\$ 51,200$ & $\$ 144,800$ \\
\hline
\end{tabular}


Reciprocating Pump

$1^{\text {st }}$ Quarter 1998 dollars

\begin{tabular}{|c|c|c|c|c|c|}
\hline $\begin{array}{c}\text { Capacity } \\
\text { (Gallons/ } \\
\text { minute) }\end{array}$ & $\begin{array}{c}\text { Driver } \\
\text { Power } \\
\text { (Horse- } \\
\text { power) }\end{array}$ & $\begin{array}{c}\text { Purchased } \\
\text { Equipment } \\
\text { Cost } \\
\mathbf{( \$ )}\end{array}$ & $\begin{array}{c}\text { Installed } \\
\text { Cost } \\
\mathbf{( \$ )}\end{array}$ & $\begin{array}{c}\text { Triplex } \\
\text { Purchased } \\
\text { Equipment } \\
\text { Cost } \\
\mathbf{( \$ )}\end{array}$ & $\begin{array}{c}\text { Installed } \\
\text { Cost } \\
\mathbf{( \$ )}\end{array}$ \\
\hline 25 & 2 & $\$ 4,100$ & $\$ 10,600$ & $\$ 7,700$ & $\$ 15,500$ \\
\hline 50 & 5 & $\$ 7,000$ & $\$ 14,600$ & $\$ 13,800$ & $\$ 22,700$ \\
\hline 100 & 7.5 & $\$ 8,800$ & $\$ 17,800$ & $\$ 17,900$ & $\$ 28,200$ \\
\hline 200 & 15 & $\$ 13,100$ & $\$ 22,500$ & $\$ 27,900$ & $\$ 38,600$ \\
\hline 300 & 25 & $\$ 17,600$ & $\$ 28,800$ & $\$ 38,700$ & $\$ 51,200$ \\
\hline 400 & 30 & $\$ 19,600$ & $\$ 31,000$ & $\$ 43,500$ & $\$ 56,200$ \\
\hline 500 & 40 & $\$ 23,100$ & $\$ 34,700$ & $\$ 52,300$ & $\$ 65,300$ \\
\hline 600 & 50 & $\$ 26,300$ & $\$ 38,100$ & $\$ 60,300$ & $\$ 73,400$ \\
\hline 700 & 60 & $\$ 29,200$ & $\$ 43,700$ & $\$ 67,800$ & $\$ 83,700$ \\
\hline 800 & 60 & $\$ 29,200$ & $\$ 43,700$ & $\$ 67,800$ & $\$ 83,800$ \\
\hline 900 & 75 & $\$ 33,300$ & $\$ 48,100$ & $\$ 78,200$ & $\$ 94,500$ \\
\hline 1,000 & 75 & $\$ 33,300$ & $\$ 48,200$ & $\$ 78,200$ & $\$ 94,500$ \\
\hline
\end{tabular}

Vacuum Pump

$1^{\text {st }}$ Quarter 1998 dollars

\begin{tabular}{|c|c|r|r|}
\hline $\begin{array}{c}\text { Capacity } \\
\text { (Gallons/ } \\
\text { minute) }\end{array}$ & Stages & $\begin{array}{r}\text { Purchased } \\
\text { Equipment } \\
\text { Cost } \\
\mathbf{( \$ )}\end{array}$ & $\begin{array}{c}\text { Installed } \\
\text { Cost } \\
\mathbf{( \$ )}\end{array}$ \\
\hline 30 & 1 & $\$ 4,100$ & $\$ 18,600$ \\
\hline 75 & 1 & $\$ 6,400$ & $\$ 21,100$ \\
\hline 150 & 1 & $\$ 8,900$ & $\$ 24,000$ \\
\hline 200 & 1 & $\$ 11,500$ & $\$ 26,900$ \\
\hline 300 & 1 & $\$ 16,200$ & $\$ 32,300$ \\
\hline 400 & 1 & $\$ 20,800$ & $\$ 37,100$ \\
\hline 500 & 1 & $\$ 25,200$ & $\$ 41,800$ \\
\hline 600 & 1 & $\$ 29,500$ & $\$ 46,300$ \\
\hline 700 & 1 & $\$ 33,700$ & $\$ 50,800$ \\
\hline 30 & 2 & $\$ 6,100$ & $\$ 20,600$ \\
\hline 75 & 2 & $\$ 8,500$ & $\$ 23,200$ \\
\hline 150 & 2 & $\$ 11,000$ & $\$ 26,100$ \\
\hline 200 & 2 & $\$ 13,600$ & $\$ 29,000$ \\
\hline 300 & 2 & $\$ 18,500$ & $\$ 34,600$ \\
\hline 400 & 2 & $\$ 22,900$ & $\$ 39,200$ \\
\hline 500 & 2 & $\$ 27,100$ & $\$ 43,700$ \\
\hline 600 & 2 & $\$ 31,000$ & $\$ 47,800$ \\
\hline 700 & 2 & $\$ 34,800$ & $\$ 51,900$ \\
\hline
\end{tabular}


Reciprocating Compressor

$1^{\text {st }}$ Quarter 1998 dollars

\begin{tabular}{|c|c|c|r|r|}
\hline Stages & $\begin{array}{c}\text { Actual } \\
\text { Capacity } \\
\text { (Cubic feet/ } \\
\text { minute) }\end{array}$ & $\begin{array}{r}\text { Driver Power } \\
\text { (Horsepower) }\end{array}$ & $\begin{array}{r}\text { Purchased } \\
\text { Equipment } \\
\text { Cost } \\
\mathbf{( \$ )}\end{array}$ & \multicolumn{1}{|c|}{$\begin{array}{c}\text { Installed Cost } \\
\mathbf{( \$ )}\end{array}$} \\
\hline 1 & 250 & 40 & $\$ 186,200$ & $\$ 245,500$ \\
\hline 1 & 500 & 75 & $\$ 233,700$ & $\$ 300,300$ \\
\hline 1 & 1,000 & 125 & $\$ 301,700$ & $\$ 380,400$ \\
\hline 1 & 5,000 & 600 & $\$ 589,600$ & $\$ 717,500$ \\
\hline 1 & 10,000 & 1,250 & $\$ 810,400$ & $\$ 970,700$ \\
\hline 1 & 25,000 & 3,000 & $\$ 1,891,500$ & $\$ 2,139,000$ \\
\hline 1 & 50,000 & 5,500 & $\$ 4,024,800$ & $\$ 4,469,700$ \\
\hline 1 & 60,000 & 7,000 & $\$ 4,837,400$ & $\$ 5,354,000$ \\
\hline 3 & 250 & 100 & $\$ 297,000$ & $\$ 358,800$ \\
\hline 3 & 500 & 150 & $\$ 355,400$ & $\$ 422,200$ \\
\hline 3 & 1,000 & 300 & $\$ 431,400$ & $\$ 509,700$ \\
\hline 3 & 5,000 & 1,500 & $\$ 822,400$ & $\$ 932,300$ \\
\hline 3 & 10,000 & 3,000 & $\$ 1,489,700$ & $\$ 1,646,100$ \\
\hline 3 & 25,000 & 7,000 & $\$ 3,794,300$ & $\$ 4,135,200$ \\
\hline 3 & 35,000 & 10,000 & $\$ 5,519,000$ & $\$ 6,038,600$ \\
\hline 3 & 250 & 800 & $\$ 389,400$ & $\$ 467,200$ \\
\hline 3 & 500 & 1,500 & $\$ 534,100$ & $\$ 627,400$ \\
\hline 3 & 1,000 & 3,000 & $\$ 1,080,700$ & $\$ 1,211,500$ \\
\hline 3 & 5,000 & 15,000 & $\$ 3,750,700$ & $\$ 4,211,800$ \\
\hline 3 & 7,000 & 22,500 & $\$ 4,712,700$ & $\$ 5,317,700$ \\
\hline
\end{tabular}




\section{Centrifugal Compressor}

$1^{\text {st }}$ Quarter 1998 dollars

\begin{tabular}{|c|c|c|r|r|}
\hline Stages & $\begin{array}{c}\text { Actual } \\
\text { Capacity } \\
\text { (Cubic feet/ } \\
\text { minute) }\end{array}$ & $\begin{array}{r}\text { Driver Power } \\
\text { (Horsepower) }\end{array}$ & $\begin{array}{r}\text { Purchased } \\
\text { Equipment } \\
\text { Cost } \\
\mathbf{( \$ )}\end{array}$ & $\begin{array}{c}\text { Installed Cost } \\
\mathbf{( \$ )}\end{array}$ \\
\hline 4 & 500 & 60 & $\$ 595,400$ & $\$ 702,700$ \\
\hline 4 & 1,000 & 125 & $\$ 626,400$ & $\$ 749,300$ \\
\hline 4 & 5,000 & 600 & $\$ 719,700$ & $\$ 907,100$ \\
\hline 4 & 10,000 & 1,250 & $\$ 1,114,800$ & $\$ 1,339,000$ \\
\hline 4 & 50,000 & 6,000 & $\$ 2,699,800$ & $\$ 3,247,700$ \\
\hline 4 & 100,000 & 12,000 & $\$ 5,275,800$ & $\$ 6,142,000$ \\
\hline 4 & 150,000 & 17,000 & $\$ 8,722,600$ & $\$ 9,735,100$ \\
\hline 4 & 200,000 & 25,000 & $\$ 9,627,600$ & $\$ 10,980,400$ \\
\hline 9 & 500 & 125 & $\$ 975,600$ & $\$ 1,066,700$ \\
\hline 9 & 1,000 & 250 & $\$ 1,011,200$ & $\$ 1,118,500$ \\
\hline 9 & 5,000 & 1,250 & $\$ 1,146,600$ & $\$ 1,286,000$ \\
\hline 9 & 10,000 & 2,500 & $\$ 1,889,300$ & $\$ 2,060,500$ \\
\hline 8 & 50,000 & 12,000 & $\$ 4,821,600$ & $\$ 5,356,700$ \\
\hline 8 & 100,000 & 25,000 & $\$ 12,444,800$ & $\$ 13,267,000$ \\
\hline 7 & 150,000 & 37,500 & $\$ 18,991,500$ & $\$ 19,966,000$ \\
\hline 7 & 200,000 & 50,000 & $\$ 19,394,300$ & $\$ 20,624,400$ \\
\hline 9 & 500 & 1,750 & $\$ 1,446,400$ & $\$ 1,548,200$ \\
\hline 9 & 1,000 & 3,500 & $\$ 1,560,500$ & $\$ 1,680,300$ \\
\hline 9 & 5,000 & 16,000 & $\$ 2,258,600$ & $\$ 2,527,000$ \\
\hline 9 & 10,000 & 32,500 & $\$ 4,053,700$ & $\$ 4,467,800$ \\
\hline 9 & 15,000 & 50,000 & $\$ 5,171,000$ & $\$ 5,718,400$ \\
\hline
\end{tabular}

Centrifugal Fan

$1^{\text {st }}$ Quarter 1998 dollars

\begin{tabular}{|c|r|r|}
\hline $\begin{array}{c}\text { Actual Capacity } \\
\text { (Gallons/ } \\
\text { minute) }\end{array}$ & $\begin{array}{c}\text { Purchased } \\
\text { Equipment } \\
\text { Cost } \\
\text { (\$) }\end{array}$ & $\begin{array}{c}\text { Installed Cost } \\
\mathbf{( \$ )}\end{array}$ \\
\hline 700 & $\$ 1,100$ & $\$ 7,000$ \\
\hline 1,500 & $\$ 1,100$ & $\$ 7,400$ \\
\hline 5,000 & $\$ 1,800$ & $\$ 9,800$ \\
\hline 10,000 & $\$ 2,500$ & $\$ 13,100$ \\
\hline 25,000 & $\$ 6,700$ & $\$ 27,900$ \\
\hline 50,000 & $\$ 13,300$ & $\$ 49,900$ \\
\hline 75,000 & $\$ 19,900$ & $\$ 64,900$ \\
\hline 100,000 & $\$ 31,400$ & $\$ 93,400$ \\
\hline 150,000 & $\$ 44,600$ & $\$ 126,500$ \\
\hline
\end{tabular}


Rotary Blower

$1^{\text {st }}$ Quarter 1998 dollars

\begin{tabular}{|c|r|r|}
\hline $\begin{array}{c}\text { Actual Capacity } \\
\text { (Gallons/ } \\
\text { minute) }\end{array}$ & $\begin{array}{c}\text { Purchased } \\
\text { Equipment } \\
\text { Cost } \\
\mathbf{( \$ )}\end{array}$ & $\begin{array}{c}\text { Installed Cost } \\
\mathbf{( \$ )}\end{array}$ \\
\hline 100 & $\$ 4,800$ & $\$ 11,500$ \\
\hline 500 & $\$ 10,400$ & $\$ 19,100$ \\
\hline 1,000 & $\$ 15,000$ & $\$ 24,900$ \\
\hline 2,000 & $\$ 22,000$ & $\$ 34,800$ \\
\hline 3,000 & $\$ 28,100$ & $\$ 44,400$ \\
\hline 4,000 & $\$ 36,700$ & $\$ 54,600$ \\
\hline
\end{tabular}

Gas Turbine

$1^{\text {st }}$ Quarter 1998 dollars

\begin{tabular}{|c|r|r|}
\hline $\begin{array}{c}\text { Power Output } \\
\text { (Horsepower) }\end{array}$ & $\begin{array}{r}\text { Purchased } \\
\text { Equipment Cost } \\
\mathbf{( \$ )}\end{array}$ & $\begin{array}{c}\text { Installed Cost } \\
\mathbf{( \$ )}\end{array}$ \\
\hline 1,000 & $\$ 476,200$ & $\$ 565,200$ \\
\hline 5,000 & $\$ 1,254,100$ & $\$ 1,376,400$ \\
\hline 10,000 & $\$ 1,903,000$ & $\$ 2,051,300$ \\
\hline 50,000 & $\$ 9,639,300$ & $\$ 9,975,400$ \\
\hline 100,000 & $\$ 16,148,100$ & $\$ 16,738,600$ \\
\hline 150,000 & $\$ 21,837,300$ & $\$ 22,659,400$ \\
\hline 200,000 & $\$ 27,052,000$ & $\$ 28,056,000$ \\
\hline 250,000 & $\$ 31,940,100$ & $\$ 33,192,400$ \\
\hline 300,000 & $\$ 36,583,000$ & $\$ 37,998,000$ \\
\hline 350,000 & $\$ 41,031,000$ & $\$ 42,609,000$ \\
\hline 370,000 & $\$ 42,764,000$ & $\$ 44,407,000$ \\
\hline
\end{tabular}


Steam Turbine

$1^{\text {st }}$ Quarter 1998 dollars

\begin{tabular}{|c|r|r|}
\hline $\begin{array}{c}\text { Power Output } \\
\text { (Horsepower) }\end{array}$ & $\begin{array}{r}\text { Purchased } \\
\text { Equipment Cost } \\
\mathbf{( \$ )}\end{array}$ & $\begin{array}{c}\text { Installed Cost } \\
\mathbf{( \$ )}\end{array}$ \\
\hline 10 & $\$ 19,100$ & $\$ 36,000$ \\
\hline 50 & $\$ 25,200$ & $\$ 46,500$ \\
\hline 100 & $\$ 28,500$ & $\$ 53,600$ \\
\hline 500 & $\$ 37,700$ & $\$ 108,800$ \\
\hline 950 & $\$ 42,100$ & $\$ 126,700$ \\
\hline 1,000 & $\$ 85,000$ & $\$ 169,800$ \\
\hline 2,500 & $\$ 269,000$ & $\$ 364,400$ \\
\hline 5,000 & $\$ 575,000$ & $\$ 688,000$ \\
\hline 7,500 & $\$ 781,400$ & $\$ 907,900$ \\
\hline 10,000 & $\$ 971,400$ & $\$ 1,106,600$ \\
\hline 15,000 & $\$ 1,320,100$ & $\$ 1,477,100$ \\
\hline 20,000 & $\$ 1,641,100$ & $\$ 1,825,200$ \\
\hline 30,000 & $\$ 2,230,200$ & $\$ 2,447,300$ \\
\hline
\end{tabular}

